\title{
CURVATURE OF YANG-MILLS MODULI SPACES NEAR THE BOUNDARY, I
}

\author{
DAVID GROISSER
}

\section{INTRODUCTION}

The topology of the moduli space $\mathcal{M}$ of self-dual connections ("instantons") on a principal $S U(2)$-bundle over a compact Riemannian 4-manifold $(M, g)$ has been studied for a number of years, thanks largely to the pioneering work of Donaldson ([D1]). Relatively speaking, the study of the geometry of $\mathcal{M}$ with respect to its natural Riemannian metric g-the " $L^{2}$ metric"-is still in its infancy. There are only two examples in which $\mathbf{g}$ has been computed explicitly and in which the geometry is completely understood: the moduli spaces of $k=1$ instantons over $\mathbb{S}^{4}$ ([GP1, DMM, H]) and $\mathbb{C P}^{2}$ ([G1, Ko]). (In this paper $\mathbb{S}^{4}$ and $\mathbb{C P}^{2}$ are always given their standard metrics.) In [GP2] many features of the geometry seen in these examples were shown to be present for all 1-instanton moduli spaces over compact, positive-definite, simply-connected 4-manifolds, and in [D2], Donaldson was able to deduce some geometric information under even more general hypotheses. However, the two basic examples exhibited more structure than they seemed to have any right to; there were several geometric features for which neither proofs nor counterexamples existed in the more general setting. In particular the boundary $\partial \overline{\mathcal{M}}$ of the completion of $\mathcal{M}$ was found to be totally geodesic, and $\mathrm{g}$ was found to be more differentiable at the boundary in these examples than the authors of [GP2] were able to prove in general.

This article is a step towards understanding the general problem of differentiability at the boundary and the geometry of the collar. In pursuing

Research partially supported by N. S. F. grant DMS-8905211 
this problem the author discovered a surprising cancellation phenomenon, discussed below, relating to the sectional curvature of $\mathcal{M}$. It also proved useful to develop a new method for constructing parametrices for certain Green operators, and to refine an estimate of Donaldson concerning the decay rate of curvatures of concentrated instantons.

Throughout this paper $M$ denotes a compact, simply connected, oriented, positive-definite 4-manifold, and $\mathcal{M}$ the space of 1-instantons over $M$. Under these topological assumptions $\mathcal{M}$ is generically a 5 -dimensional manifold with singularities. $\mathcal{M}$ contains a 'collar' region $\mathcal{M}_{\lambda_{0}}$ diffeomorphic (non- canonically) to $\left(0, \lambda_{0}\right) \times M$ for some $\lambda_{0}>0$; the diffeomorphism $\Psi^{-1}$ assigns to an instanton its scale $\lambda \in\left(0, \lambda_{0}\right)$ and its center $p \in M$ (see [D1]). We will assume some familiarity with these notions and with the definitions and basic properties of the $L^{2}$ metric as discussed in [GP1] and [GP2]. Certain properties are true only for $\lambda_{0}$ sufficiently small; we will always assume, without explicit mention, that $\lambda_{0}$ has been chosen small enough. The following paraphrases some basic results of [GP2].

Theorem 0.1 (Theorems II-IV of [GP2]). Let $\overline{\mathcal{M}}$ and $\overline{\mathcal{M}_{\lambda_{0}}}$ denote the metric completions of $\mathcal{M}$ and $\mathcal{M}_{\lambda_{0}}$ respectively. Then the collar map $\Psi^{-1}$ extends to a diffeomorphism $\overline{\mathcal{M}_{\lambda_{0}}} \cong\left[0, \lambda_{0}\right] \times M$, and the inclusion $\mathcal{M}_{\lambda_{0}} \hookrightarrow$ $\mathcal{M}$ induces an identification $\{0\} \times M \cong \partial \overline{\mathcal{M}}$. As $\lambda \rightarrow 0$, the metric $\mathrm{g}$ is asymptotic, in a $C^{0}$ sense, to a product:

$$
\Psi^{*} \mathbf{g} \sim 4 \pi^{2}\left(2 d \lambda^{2} \oplus g\right)
$$

Thus if we define $\bar{\lambda}[A]=\left(8 \pi^{2}\right)^{-1 / 2} \operatorname{dist}([A], \partial \overline{\mathcal{M}})$ for $[A] \in \mathcal{M}$, then

$$
\frac{\bar{\lambda}}{\lambda} \rightarrow 1
$$

uniformly in $[A]$ as $\lambda \rightarrow 0$.

This product structure does not extend to arbitrary derivatives of $\mathbf{g}$. In particular, in the $\mathbb{S}^{4}$ and $\mathbb{C P}^{2}$ examples, the curvature of $\mathcal{M}$ extends continuously to the boundary, and its boundary values are not the curvature tensor of a cylinder (although the curvature in directions tangent to the boundary is $\left(4 \pi^{2}\right)^{-1}$ times that of $(M, g)$, as one would expect from (0.1). It is worthwhile 
to compare the explicit formulas for $\mathrm{g}$ found in these examples ( $c f$. [GP3] and [G1]). In each case $\mathcal{M}$ has an 'origin' on the complement of which

$$
\Psi^{*} \mathrm{~g}=4 \pi^{2}\left(2 f(\lambda) d \lambda^{2} \oplus h(\lambda) g\right)
$$

for certain functions $f$ and $h$ which approach 1 as $\lambda \rightarrow 0$; see (0.3-0.6) below. Note that $f$ is not $C^{2}$ at the boundary in either case, so it seems paradoxical that the curvature of $\mathcal{M}$ manages to be continuous there.

The solution to this paradox lies in understanding the smooth structure on $\overline{\mathcal{M}}$. In [GP2] it was wrongly stated that the smooth structure on $\overline{\mathcal{M}}$ induced by the collar map $\Psi$ is independent of the choices involved in defining $\Psi$, and therefore that $\overline{\mathcal{M}}$ has a natural smooth structure. Indeed, in the examples above, $\overline{\mathcal{M}}$ does have a natural smooth structure, but it is not the one given by the collar map from which (0.3-0.6) were derived. (It should also be noted that in these equations, $\lambda$ is the radius of the smallest ball containing exactly half the Yang-Mills action; Donaldson's $\lambda$, which is the one used in Theorem 0.1, smooths out the cutoff in a non-canonical way.) The correct smooth structure is given not by declaring $\lambda$ to be smooth on $\overline{\mathcal{M}_{\lambda_{0}}}$-i.e., by using the extension of $\Psi$ to $\left[0, \lambda_{0}\right) \times M$ as an attaching map-but by declaring $\bar{\lambda}$, the (normalized) distance to the boundary, to be smooth. This is sensible only because, as the next theorem shows, $\bar{\lambda}$ is smooth in $\mathcal{M}_{\lambda_{0}}$.

DeFinition 0.1. For $\lambda_{0}$ sufficiently small define

$$
\bar{\Psi}^{-1}: \mathcal{M}_{\lambda_{0}} \rightarrow(0, \infty) \times M \quad \text { by } \quad \bar{\Psi}^{-1}([A])=(\bar{\lambda}([A]), \bar{p}([A]))
$$

where $\bar{\lambda}([A])$ is the normalized distance-to-the- boundary as in Theorem 0.1 and $\bar{p}([A])$ is the closest point in $\partial \overline{\mathcal{M}} \cong M$ to $[A]$. (Note that $\bar{p}([A])$ is unique for $\lambda_{0}$ as in Theorem 0.1 , and $\bar{\Psi}^{-1}$ is $1-1$ for such $\lambda_{0}$.) On any set $(0, \epsilon) \times M \subset \operatorname{image}\left(\bar{\Psi}^{-1}\right)$, let $\bar{\Psi}$ denote the inverse of $\bar{\Psi}^{-1}$.

Theorem 0.2. Let $M$ be $\mathbb{S}^{4}$ or $\mathbb{C P}^{2}$. Then $\bar{\Psi}^{-1}$ is smooth on $\mathcal{M}_{\lambda_{0}}$, so we can give $\overline{\mathcal{M}}$ the smooth structure of $\mathcal{M} \bigcup_{\bar{\Psi}}\left(\left[0, \overline{\lambda_{0}}\right) \times M\right)$ without affecting the smooth structure on the interior. With respect to this smooth structure on $\overline{\mathcal{M}}_{0}, \bar{\Psi}^{*} \mathbf{g}$ is $C^{5}$ at the boundary but not $C^{6}$. 
Proof. From [GP3, equations (5.15-16)] and [G1, equations (0.6-7)] the metric $\mathrm{g}$ takes the form $(0.2)$, where for $\mathbb{S}^{4}$ we have

$$
\begin{gathered}
f(\lambda)=\sec ^{2} \lambda\left(1+3 \tan ^{2} \lambda-3 \sec \lambda \tan ^{2} \lambda \log (\cot (\lambda / 2))\right), \\
h(\lambda)=1-\frac{3}{2} \tan ^{2} \lambda+\frac{3}{2} \cos \lambda \tan ^{4} \lambda \log (\cot (\lambda / 2)),
\end{gathered}
$$

while for $\mathbb{C P}^{2}$ we have

$$
\begin{gathered}
f(\lambda)=\frac{1+3 \lambda^{2}}{\left(1-\lambda^{2}\right)^{3}}+\frac{6+2 \lambda^{2}}{\left(1-\lambda^{2}\right)^{4}} \lambda^{2} \log (\lambda), \\
h(\lambda)=\frac{1}{2+\lambda^{2}}\left\{\frac{2-7 \lambda^{2}-\lambda^{4}}{1-\lambda^{2}}-\frac{12}{\left(1-\lambda^{2}\right)^{2}} \lambda^{4} \log (\lambda)\right\} .
\end{gathered}
$$

From (0.2) it follows that $p([A])=\bar{p}([A])$ (where $\Psi([A])=(\lambda([A]), p([A])))$ and that distance-to-the-boundary is a function of $\lambda$ alone. Specifically,

$$
\bar{\lambda}(\lambda)=\int_{0}^{\lambda} f(t)^{1 / 2} d t
$$

which is smooth for $\lambda>0$. This equation determines $\lambda$ implicitly as a function of $\bar{\lambda}$, so we may rewrite the metric in the form $4 \pi^{2}\left(2 d \bar{\lambda}^{2} \oplus \bar{h}(\bar{\lambda}) g\right)$. Using (0.7) and the formulas for $h$, one obtains the result (after some very tedious calculations). In fact, in each case we have asymptotic expansions of the form

$$
\begin{aligned}
& f(\lambda)=1+\lambda^{2}\left(a_{1} \log (\lambda)+a_{2}\right)+\lambda^{4}\left(a_{3} \log (\lambda)+a_{4}\right)+O\left(\lambda^{6} \log (\lambda)\right), \\
& h(\lambda)=1+c_{2} \lambda^{2}+\lambda^{4}\left(c_{3} \log (\lambda)+c_{4}\right)+c_{5} \lambda^{6} \log (\lambda)+O\left(\lambda^{6}\right) .
\end{aligned}
$$

One can check that for any such $f, h$, the function $\bar{h}(\bar{\lambda})$ is $C^{3}$ for $\bar{\lambda} \geq 0$. Furthermore $\bar{h}$ is $C^{4}$ if and only if $3 c_{3}-a_{1} c_{2}=0-$ a condition that holds in the two examples above-in which case $\bar{h}$ is also $C^{5}$. Finally, under this condition $\bar{h}$ is $C^{6}$ only if $c_{2}=0$, which does not happen in either of the examples.

It should be noted, however, that while the smooth structure on $\overline{\mathcal{M}}$ depends the attaching map, its restriction to $\partial \mathcal{M}$ does not; it is always the smooth structure of $M$. In addition it follows from Theorem 0.1 that the normalized distance $\bar{\lambda}$ is differentiable at the boundary whichever choice we make (though what happens just off the boundary is unclear). 
In light of Theorem 0.2, an awkward conjecture in [G1] can be replaced by a more natural one.

Conjecture 0.2 . For any $M$, the map $\bar{\Psi}^{-1}$ is smooth in the collar $\mathcal{M}_{\lambda_{0}}$. If this attaching map is used to define the smooth structure on $\overline{\mathcal{M}}$, the extension of $\mathrm{g}$ to $\overline{\mathcal{M}}$ is $C^{2}$.

A necessary condition for Conjecture 0.2 to be true is that the curvature of $\left(\mathcal{M}_{\lambda_{0}}, \mathbf{g}\right)$ be continuous up to the boundary. In this paper we find substantial evidence that this is the case.

It seems premature to conjecture more differentiability than this based only on Theorem 0.2. Loosely speaking, one finds that obtaining $C^{k}$ information about the metric $\mathrm{g}$ requires $O\left(\lambda^{k}\right)$ estimates on certain spaces $H_{A} \cong T_{[A]} \mathcal{M}$ (see (1.2) below). But the estimation scheme presented here (culminating in Proposition 5.1) is intrinsically limited from giving estimates stronger than $O\left(\lambda^{2}\right)$, and hence does not suggest any higher degree of differentiability than $C^{2}$. It is conceivable that the examples in Theorem 0.2 exhibit more differentiability because of symmetry; e.g. because both $\mathbb{S}^{4}$ and $\mathbb{C P}^{2}$ are Einstein. However, it is also possible that refined estimates would show more differentiability in the general case.

\section{Statement of Results and Strategy of the Proofs}

1.1. Results. Our first theorem requires $(M, g)$ to satisfy a certain curvature condition.

Definition 1.1. Let $R$ and $W^{-}$be the scalar curvature and anti-self-dual Weyl tensor of $(M, g)$. Define an operator $\mathcal{W}^{-}$on two-forms by $\mathcal{W}_{-}\left(\theta^{i} \wedge \theta^{j}\right)=$ $\frac{1}{2}\left(W^{-}\right)^{i j}{ }_{k l} \theta^{k} \wedge \theta^{l}$; this preserves the subspace of anti-self-dual two forms. We say that $(M, g)$ satisfies the $\Lambda_{-}^{2}$ curvature condition at $p \in M$ if the operator

$$
\frac{R}{6} \mathrm{Id}-2 \mathcal{W}_{-}: \bigwedge_{-}^{2} T_{p}^{*} M \rightarrow \bigwedge_{-}^{2} T_{p}^{*} M
$$

is positive-definite.

Frequently when discussing moduli spaces of self-dual connections one assumes that a very similar operator, $\frac{R}{3} \operatorname{Id}-2 \mathcal{W}_{-}$, is positive-definite; this ensures that the moduli space is a manifold on the complement of the reducible 
connections. We do not need the latter assumption here since we are only concerned with the collar, which is always a manifold. Nonetheless the similarity between the two curvature conditions is striking.

The purpose of the $\Lambda_{-}^{2}$ curvature condition is that it ensures a strong decay rate for the curvature of a concentrated instanton, which we use in $\S 3$ for several important estimates that we apply over and over again. Donaldson established in [D1] that such curvatures decay at least as fast as $\lambda^{2-\delta} r^{-(4-\delta)}$ for any $\delta>0$. By carefully examining his argument, and observing that the $\bigwedge_{-}^{2}$ curvature condition is exactly what is needed to eliminate the $\delta$ (Theorem 2.2 ), we sharpen the decay rate to $\lambda^{2} r^{-4}$. This estimate is proven in $\S 4$.

We can now state our main theorems.

Theorem 1.1. Let $(M, g)$ be a compact, simply connected, oriented, positivedefinite 4-manifold, and $\mathcal{M}$ the space of 1-instantons over $M$. Assume that $(M, g)$ satisfies the $\Lambda_{-}^{2}$ curvature condition. Then for $\lambda_{0}$ sufficiently small the sectional curvature of $\left(\mathcal{M}_{\lambda_{0}}, \mathbf{g}\right)$ is bounded above and below.

It seems likely that the methods here could be refined to prove continuity of the curvature, but the problem of passing from $C^{0}$ curvature to $C^{2}$ metric would remain.

It is not actually necessary to assume the $\Lambda_{-}^{2}$ curvature condition on all of $M$ in Theorem 1.1 to reach a conclusion. If we assume only that it is satisfied in a neighborhood $U$ of some point $p$, our methods show that the same conclusion holds for the corresponding region of the collar.

We can say a good deal more about the curvature in directions tangent to the boundary, even without the $\Lambda_{-}^{2}$ curvature condition. After fixing a collar map, $\mathcal{M}_{\lambda_{0}}$ is foliated by submanifolds $M_{\lambda}$ of constant $\lambda$, with the inclusion map $j_{\lambda}: M \rightarrow \mathcal{M}$ giving a diffeomorphism onto $M_{\lambda}$. We denote by Riem ${ }_{\mathcal{M}}^{\text {tan }}$ the restriction of the Riemann tensor of $\left(\mathcal{M}_{\lambda_{0}}, \mathbf{g}\right)$ to this 'tangential' foliation and by $\operatorname{Riem}_{M}$ the Riemann tensor of $(M, g)$.

Theorem 1.2. Assume all hypotheses of Theorem 1.1 except the $\bigwedge_{-}^{2}$ curvature condition. Then $\operatorname{Riem}_{\mathcal{M}}^{\mathrm{tan}}$ extends continuously to $\overline{\mathcal{M}_{\lambda_{0}}}$, and the limit 
depends only on $\operatorname{Riem}_{M}$. Specifically,

$$
\left.\operatorname{Riem}_{\mathcal{M}}^{\mathrm{tan}}\right|_{\partial \mathcal{M}}:=\lim _{\lambda \rightarrow 0} j_{\lambda}^{*} \operatorname{Riem}_{\mathcal{M}}^{\mathrm{tan}}=L\left(\operatorname{Riem}_{M}\right)
$$

for some geometrically natural endomorphism $L$.

By 'geometrically natural' we mean that $L$ is induced from an $S O(4)$ equivariant endomorphism of the subspace of $\mathbb{R}^{4} \otimes \mathbb{R}^{4} \otimes \mathbb{R}^{4} \otimes \mathbb{R}^{4}$ consisting of tensors with the symmetries of a Riemann tensor.

The map $L$ in Theorem 1.2 is computed in the sequel to this paper [G2]; we find that $L$ is $\left(4 \pi^{2}\right)^{-1}$ times the identity. This provides a plausibility argument for the following conjecture.

ConjeCture 1.2. $\partial \overline{\mathcal{M}_{\lambda_{0}}}$ is a totally geodesic submanifold of $\overline{\mathcal{M}_{\lambda_{0}}}$.

The argument is as follows: if we assume that the metric is $C^{2}$ at the boundary we can apply the Gauss equations

$$
\left.\operatorname{Riem}_{\mathcal{M}}^{\tan }\right|_{\partial \mathcal{M}}=\operatorname{Riem}_{\partial \mathcal{M}}+h \wedge h,
$$

where $h$ is the second fundamental form of the boundary. The boundary is totally geodesic if and only if $h$ is identically zero. Since the metric on $\partial \overline{\mathcal{M}}$ is simply $4 \pi^{2} g$ under the natural identification of $\partial \overline{\mathcal{M}}$ with $M$ (see Theorem 0.1 ), we always have $\operatorname{Riem}_{\partial \mathcal{M}}=\left(4 \pi^{2}\right)^{-1} \operatorname{Riem}_{M}$. Hence, using $L=\left(4 \pi^{2}\right)^{-1} \mathrm{Id}$ in Theorem 1.2, we find $h \wedge h=0$. This implies that $h$ has rank $\leq 1$; i.e. there is a 1 -form $\theta$ (possibly zero) on $\partial \mathcal{M}$ such that $h=\theta \otimes \theta$. Note that generically the second fundamental form of a 4-dimensional submanifold of a 5-manifold has rank 4; it seems unlikely that for all $(M, g)$ the second fundamental form of $\partial \overline{\mathcal{M}}$ has rank $\leq 1$ unless it is identically zero, as it is for $\mathbb{S}^{4}$ and $\mathbb{C P}^{2}$. In fact, if one assumes that $h$ depends on $\operatorname{Riem}_{M}$ in a geometrically natural way, it is easy to show that if $\operatorname{rank}(h)$ is always $\leq 1$ we must have $h \equiv 0$.

1.2. Strategy. Our approach to proving Theorems (1.1-1.2) will rely on the expression derived in [GP1] for the sectional curvature of $\mathcal{M}$ at a point $[A]$ represented by a self-dual connection $A$. To review this formula, recall that the tangent space $T_{[A]} M$ is naturally isomorphic to the harmonic space

$$
H_{A}=\left\{X \in \Omega^{1}(\operatorname{Ad} P) \mid d^{A^{*}} X=0 \in \Omega^{0}(\operatorname{Ad} P) \text { and } d_{-}^{A} X=0 \in \Omega_{-}^{2}(\operatorname{Ad} P)\right\} \text {, }
$$


where $P \rightarrow M$ is the principal $S U(2)$-bundle with Pontryagin index $1, d^{A}$ is convariant exterior derivative, and the minus sign denotes projection $p_{-}$to the space of anti-self-dual two-forms. Alternatively, $H_{A}=\operatorname{ker}\left(\Delta_{1}^{A}\right)$, where

$$
\Delta_{1}^{A}=d^{A}\left(d^{A}\right)^{*}+2\left(d_{-}^{A}\right)^{*} d_{-}^{A} .
$$

We introduce notation for two universal bilinear operations, $\{\cdot, \cdot\}:($ Ad $P \otimes$ $\left.T^{*} M\right) \otimes\left(\operatorname{Ad} P \otimes T^{*} M\right) \rightarrow$ Ad $P$ and $[\cdot, \cdot]:\left(\right.$ Ad $\left.P \otimes T^{*} M\right) \otimes\left(\operatorname{Ad} P \otimes T^{*} M\right) \rightarrow$ Ad $P \otimes \wedge^{2} T^{*} M$. In terms of a local orthonormal basis $\left\{\theta^{a}\right\}$ of $T^{*} M$, the antisymmetric pairing $\{\cdot, \cdot\}$ is defined by

$$
\{X, Y\}=\left[X_{a}, Y_{a}\right]
$$

(Lie-algebra bracket); the symmetric pairing $[\cdot, \cdot]$ is defined by

$$
[X, Y]_{-}=p_{-}\left(\left[X_{a}, Y_{b}\right] \theta^{a} \wedge \theta^{b}\right) .
$$

We also define Laplacians

$$
\Delta_{0}^{A}=d^{A^{*}} d^{A}, \quad \Delta_{-}^{A}=d_{-}^{A} d_{-}^{A^{*}},
$$

which act on 0 -forms and 2-forms respectively, and are invertible in the collar; we denote the inverses by $G_{0}^{A}$ and $G_{-}^{A}$. If $X, Y \in H_{A}$ are $L^{2}$-orthonormal, the sectional curvature of $(\mathcal{M}, \mathbf{g})$ in the plane spanned by $X$ and $Y$ is then

$$
\begin{aligned}
\sigma_{A}(X, Y)=3\langle\{X, Y\} & \left., G_{0}^{A}\{X, Y\}\right\rangle \\
& -\left\langle[X, Y]_{-}, G_{-}^{A}[X, Y]_{-}\right\rangle+\left\langle[X, X]_{-}, G_{-}^{A}[Y, Y]_{-}\right\rangle
\end{aligned}
$$

where $\langle\cdot, \cdot\rangle$ denotes $L^{2}$ inner product.

Now in general it can be shown that as $\lambda \rightarrow 0$, the eigenvalues of $\Delta_{-}^{A}$ are bounded away from zero ( $c f$. [L, Ch. VI $\S 3])$, while $\Delta_{0}^{A}$ has three eigenvalues commensurate with $\lambda^{2}$ and the remainder bounded away from zero (see Proposition 8.3). To understand the asymptotic behavior of the individual terms in (1.3), it is useful to examine the case $M=\mathbb{S}^{4}$, where we know that the curvature has a finite limit as $\lambda \rightarrow 0$. At first glance it would seem that, since $G_{-}^{A}$ is uniformly bounded, the last two terms in (1.3) must each have a finite limit; therefore so must the first term, despite the small eigenvalues of $\Delta_{0}^{A}$. 
This naive analysis is flawed because the $L^{2}$-norms of $\{X, Y\}$ and $[X, Y]$ are not bounded by the $L^{2}$-norms of $X$ and $Y$. In fact, for $\mathbb{S}^{4}$ it can be shown that all three terms in (1.3) behave as $\lambda^{-2}$ as $\lambda \rightarrow 0$, but the singularities cancel. These conspiratorial cancellations are all the more remarkable in view of the unrelated origins of the terms in (1.3): the first term is the curvature of the ambient space $\mathcal{A} / \mathcal{G}=\{$ connections $\} /$ \{gauge transformations , while the other two terms come from the second fundamental form of the embedding $\mathcal{M} \hookrightarrow \mathcal{A} / \mathcal{G}$.

We will prove Theorem 0.3 by showing that this cancellation phenomenon is quite general. The first step in the proof, discussed in $\S 2$, is to understand why the cancellation 'ought' to occur. For this we replace the true tangent space $H_{A}$ from which $X$ and $Y$ in (1.3) are drawn by an approximate tangent space $\tilde{H}_{A}$ introduced in [GP2]. For $X, Y \in \tilde{H}_{A}$ we construct local expressions which approximate the global objects $G_{0}^{A}\{X, Y\}$ and $G_{-}^{A}[X, Y]_{-}$up to remainders that are small with respect to certain weighted $L^{p}$-norms. These local expressions, when plugged into (1.3), cause each of the three terms to diverge as $\lambda^{-2}$. However the inner products defining these three terms actually cancel pointwise, thanks to a quadrilinear identity involving inner products of Ad $P$ valued 1 -forms (Lemma 1.5). The remainder terms give only a bounded-and, in certain cases, calculable-contribution to the curvature.

The second and harder step in the proof is to show that the replacement of $H_{A}$ by $\tilde{H}_{A}$ alters (1.3) only by a bounded amount. This requires showing that the operator $\left.\left(\mathrm{Id}-\pi_{A}\right)\right|_{\tilde{H}_{A}}$, where

$$
\pi_{A}: \Omega^{1}(\operatorname{Ad} P) \rightarrow H_{A}
$$

is the $L^{2}$-orthogonal projection, is sufficiently small in appropriate norms. We are led to the study of the elliptic equation

$$
\Delta_{-}^{A} \eta=H^{0} \phi \curvearrowleft F^{A},
$$

where $\eta$ is an anti-self-dual two-form, $H^{0} \phi$ is the traceless Hessian of a function $\phi$, the two-form $F^{A}$ is the curvature of the connection $A$, and $\downarrow$ is a certain bilinear operation that pairs a traceless symmetric tensor with a selfdual two-form two produce an anti-self-dual two-form (see (5.5)). From this 
equation we deduce some strong estimates on the harmonic 1- forms comprising the tangent space $H_{A}$ (Proposition 4.1). All but one of the bounds we need follow fairly easily from (1.4), given the estimates in $\S 3$ (for which we use the decay estimate proven in $\S 4$ ). For the one remaining bound (5.4), ordinary elliptic theory such as that used to prove Lemma 5.3 does not seem to suffice. Nor do standard constructions of parametrices for Green kernels, followed by convolution with these kernels; the singular nature of the family of operators in (1.4) as $\lambda \rightarrow 0$ leads to difficulties. However, since we are not trying to solve (1.4) with an arbitrary right-hand side, we avoid these difficulties by effectively constructing not a parametrix for the Green kernel, but just for the Green operator restricted to the the space consisting of the right-hand side of (1.4) for certain functions $\phi$ (essentially functions which are linear in normal coordinates based at the center of $A$ ). This result, detailed in Theorem 5.1, is rather special in certain respects, but general in others, and may be of some interest in its own right; it does not depend on any features of the connection except self-duality. The technique (detailed in $§ \S 6-7$ ) relies on a rather bizarre use of representation theory, and should apply to a wider class of equations than the one studied here.

In $\S 8$, we provide some final estimates needed for $\S 9$, where we prove Theorems $1.1-1.2$.

Throughout this paper $\|\cdot\|_{p}$ denotes the $L^{p}$ norm on sections of any normed vector bundle over $M$. We use summation convention for repeated indices, and we take the inner product on Ad $P$ to be the one induced by minus the trace form of the standard representation of $\mathfrak{s u}(2)$.

\section{Localization of THE CURVATURE FORMUla: FiRST STAGE}

The Green operators $G_{0}^{A}, G_{-}^{A}$ and the harmonic 1-forms $X, Y \in H_{A}$ and appearing in (1.3) are intrinsically non-local objects. For this reason it is difficult to extract information from the curvature formula; there is no obvious relation among the terms. For concentrated instantons, however, there is a 'localization principle': as $\lambda \rightarrow 0$, instantons on $M$ more and more closely resemble instantons on $\mathbb{R}^{4}$, after a normal-coordinate identification of a neighborhood of an instanton's center with a neighborhood of the origin in $\mathbb{R}^{4}$. This principle 
holds quite strongly as far as the curvature two-form $F^{A}$ of the instanton is concerned, but it is less clear how localization affects the harmonic spaces $H_{A}$ and the Green operators $G^{A}$. In [GP2], the author and T. Parker introduced a localized approximation $\tilde{H}_{A}$ to $H_{A}$ (see below). In this section we show how to localize the Green operators applied to the bilinear objects appearing in (1.3) when $X$ and $Y$ lie in $\tilde{H}_{A}$. This localization is, in essence, 'why' the singularities in (1.3) are able to cancel.

For the rest of this paper we fix a 'cutoff' function $b \in C_{0}^{\infty}(\mathbb{R})$ with $b(t)=1$ for $0 \leq t \leq 1, b(t)=0$ for $t \geq 2$ and $0 \leq b(t) \leq 1$ everywhere. We also fix a number $r_{0}$ such that $3 r_{0}$ is less than the injectivity radius of $(M, g)$, and a smooth, strictly positive function $\rho: M \times M \rightarrow \mathbb{R}$ such that $\rho(p, q)=\operatorname{dist}(p, q)$ whenever $\operatorname{dist}(p, q) \leq 2 r_{0}$. For each $p \in M$ we define $r_{p}(q)=\rho(p, q)$ and $\beta_{p}(q)=b\left(r_{p}(q) / r_{0}\right)$; we will usually suppress the $p$ 's from these and related notations to avoid clutter.

We also fix a collar map $\Psi^{-1}$ as in the introduction. When the letters $p, \lambda$ appear during a discussion of a connection $A$, they always denote the center and scale of $A$, respectively. In such a context, $r$ always refers to distance to the center point $p$. We use $F^{A}$, or simply $F$, to denote the curvature 2-form of $A$

There are numerous estimates in this paper. All constants appearing in inequalities, as well as constants implicit in expressions $O(\cdot)$, are independent of $A \in \mathcal{M}_{\lambda_{0}}$, and in particular of the center point $p$. We will not make explicit mention of this uniformity below; it generally follows from simple arguments based on the compactness of $M$. Our convention concerning strings of inequalities is that values of constants are continually updated as needed. The letter $c$ in an inequality always denotes a constant, subject to the above conventions.

There are also several types of covariant derivatives in this paper. If $E$ is a vector bundle associated to the orthonormal frame bundle of $M$ (e.g. $E=T M)$, there is a tensor product connection $\nabla^{A}: \Gamma(\operatorname{Ad} P \otimes E) \rightarrow \Gamma(\operatorname{Ad} P \otimes$ $\left.E \otimes T^{*} M\right)$ induced by a connection $A$ on Ad $P$ and the Levi-Civita connection on $M$. We let $\Delta^{A}$ denote the trace Laplacian $\nabla^{A^{*}} \nabla^{A}$; note that on $\Gamma(\operatorname{Ad} P)$, $d^{A}=\nabla^{A}$ and $\Delta_{0}^{A}=\Delta^{A}$. (The Laplacians $\Delta_{0}^{A}, \Delta_{1}^{A}$, and $\Delta_{-}^{A}$ were defined 
in §1.2.) We simply write $\nabla$ and $\Delta$ for the covariant derivative and trace Laplacian on $E$. Frequently we will suppress the $A$ from the tensor product operators above; it is always clear from the object being differentiated whether these symbols refer to the tensor product connection or simply the Levi-Civita connection.

To define the approximate tangent space we require some notation. Given $\mathbf{a} \in T_{p} M$, let $f_{\mathbf{a}}$ be the unique function on $B_{2 r_{0}}(p)$ (the ball of radius $2 r_{0}$ centered at $p$ ) such that (i) $f_{\mathrm{a}}$ is a homogeneous linear polynomial in some (and hence any) normal coordinate system based at $p$, and (ii) $\operatorname{grad}\left(f_{\mathbf{a}}\right)(p)=\mathbf{a}$. We extend these functions and the (squared) distance function to all of $M$ by setting

$$
\begin{aligned}
\phi^{\prime} & =\frac{1}{2} \beta^{3} r^{2}, \\
\phi_{\mathrm{a}} & =\beta^{3} f_{\mathrm{a}} .
\end{aligned}
$$

DEFINITION 2.1. The approximate tangent space at a self-dual connection $A \in$ $\mathcal{M}_{\lambda_{0}}$ with center $p$ is the subspace of $\Omega^{1}(\operatorname{Ad} P)$ defined by

$$
\tilde{H}_{A}=\left\{\iota_{Z} F^{A} \mid Z=\operatorname{grad}\left(\phi_{\mathbf{a}}+k \phi^{\prime}\right), \quad k \in \mathbb{R}, \mathbf{a} \in T_{p} M\right\} .
$$

Henceforth we will write

$$
Z_{\mathbf{a}}=\operatorname{grad} \phi_{\mathbf{a}}, \quad Z^{\prime}=\operatorname{grad} \phi^{\prime} .
$$

and, for any vector field $Z$ and self-dual connection $A$,

$$
\tilde{Z}^{A}=\iota_{Z} F^{A} .
$$

When the connection is understood from context, we simply write $\tilde{Z}$ for $\tilde{Z}^{A}$.

As $\lambda \rightarrow 0$, the vector fields $Z$ proportional to $Z^{\prime}$ correspond to directions in $T_{A} \mathcal{M}$ approximately normal to the boundary; those of the form $Z_{\text {a }}$ give the directions tangent to the boundary. This is made more precise in [GP2, §3], where it is also shown that if $Z=Z_{\mathbf{a}}+a_{0} \lambda^{-1} Z^{\prime}$, then

$$
\left\|\iota_{Z} F^{A}\right\|_{L^{2}} \rightarrow 2 \pi \sqrt{|\mathbf{a}|^{2}+2 a_{0}^{2}}
$$


as $\lambda \rightarrow 0$, and that for $\tilde{Z} \in \tilde{H}_{A},\left\|\left(I d-\pi_{A}\right) \tilde{Z}\right\|_{L^{2}}=O\left(\lambda^{1-\delta}\right)\|X\|_{L^{2}}$ for any $\delta$. Later we shall see that this estimate can be improved by a full power of $\lambda$ in the tangential direction (Proposition 5.1a).

The localization we seek for the terms in (1.3) is a consequence of the following proposition.

Proposition 2.1. Let $A \in \mathcal{M}_{\lambda_{0}}$, let $\alpha \in \Omega^{1}(\operatorname{Ad} P)$, let $Z$ be a vector field, and let $Z^{*}$ denote the image of $Z$ under the metric isomorphism from TM to $T^{*} M$. Then

$$
G_{0}^{A}\{\tilde{Z}, \alpha\}=\frac{1}{2} \iota_{Z} \alpha+G_{0}^{A}\left(R_{0}(\tilde{Z}, \alpha)\right)
$$

and

$$
G_{-}^{A}[\tilde{Z}, \alpha]_{-}=p_{-}\left(Z^{*} \wedge \alpha\right)+G_{-}^{A}\left(R_{2}(\tilde{Z}, \alpha)\right),
$$

where the remainder terms $R_{i}$ are given by

$$
\begin{aligned}
R_{0}(\tilde{Z}, \alpha)= & -\frac{1}{2}\left(\iota_{\Delta Z} \alpha-2 \iota_{\nabla_{a}} Z \nabla_{a}^{A} \alpha+\iota_{Z}\left(\Delta_{1}^{A} \alpha\right)-\iota_{Z} \operatorname{Ric}(\alpha)\right), \\
R_{2}(\tilde{Z}, \alpha)= & -\frac{1}{2}\left\{\left(p_{-}\left(\Delta Z^{*} \wedge \alpha-2 \nabla_{a} Z^{*} \wedge \nabla_{a}^{A} \alpha+Z^{*} \wedge \Delta_{1}^{A} \alpha\right)\right.\right. \\
& \left.\left.-\left(Z^{*} \wedge \operatorname{Ric}(\alpha)\right)_{-}-\mathcal{R}_{-}\left(Z^{*} \wedge \alpha\right)_{-}\right)\right\} .
\end{aligned}
$$

Here Ric and $\mathcal{R}_{-}$are pointwise endomorphisms proportional to the Riemann tensor of $(M, g)$ and whose precise form does not concern us.

When $\alpha=\tilde{W}$ this proposition reduces to the following.

Corollary 2.2. With the same notation as above, we have

$$
G_{0}^{A}\{\tilde{Z}, \tilde{W}\}=-\frac{1}{2} F(Z, W)+G_{0}^{A}\left(R_{0}(\tilde{Z}, \tilde{W})\right),
$$

and

$$
G_{-}^{A}[\tilde{Z}, \tilde{W}]_{-}=p_{-}\left(Z^{*} \wedge \tilde{W}\right)+G_{-}^{A}\left(R_{2}(\tilde{Z}, \tilde{W})\right) .
$$

The remainder $R_{i}(\tilde{Z}, \tilde{W})$ is antisymmetric in $Z$ and $W$ for $i=0$ and symmetric for $i=2$. 
Given Proposition 2.1, the only non-obvious part of the corollary is the symmetry of $\mathrm{R}_{2}(\tilde{Z}, \tilde{W})$; antisymmetry of $\mathrm{R}_{0}$ is immediate from the antisymmetry of the other two terms in (2.7). However, from the first part of Lemma 2.3 below we have

$$
p_{-}\left(Z^{*} \wedge \tilde{W}\right)=p_{-}\left(W^{*} \wedge \tilde{Z}\right),
$$

so the symmetry of $R_{2}$ follows from (2.7).

To prove Proposition 2.1 we begin with a lemma.

Lemma 2.3. Let $\mathbb{R}^{4}$ have the standard inner product orientation. Let $\left\{e_{i}\right\}$ be $a$ basis of $\mathbb{R}^{4}$ and $\left\{\theta^{i}\right\}$ the dual basis of $\left(\mathbb{R}^{4}\right)^{*}$. For $v \in \mathbb{R}^{4}$, let $v^{*}$ denote the image of $v$ under the inner-product isomorphism from $\mathbb{R}^{4}$ to $\left(\mathbb{R}^{4}\right)^{*}$. Then for all $v, w \in \mathbb{R}^{4}$ and $f \in \bigwedge_{+}^{2}\left(\mathbb{R}^{4}\right)^{*}$, the expressions

$$
p_{-}\left(v^{*} \wedge \iota_{w} f\right) \in \bigwedge_{-}^{2}\left(\mathbb{R}^{4}\right)^{*}
$$

and

$$
f\left(v, e_{i}\right) p_{-}\left(w^{*} \wedge \theta^{i}\right) \in \bigwedge_{-}^{2}\left(\mathbb{R}^{4}\right)^{*}
$$

are symmetric in $v$ and $w$.

Proof. Each of these expressions determines an $S O(4)$-equivariant map from $\mathbb{R}^{4} \otimes \mathbb{R}^{4}$ to $\operatorname{Hom}\left(\bigwedge_{+}^{2}\left(\mathbb{R}^{4}\right)^{*}, \bigwedge_{-}^{2}\left(\mathbb{R}^{4}\right)^{*}\right)$, which is $S O(4)$-isomorphic to $\operatorname{Sym}_{0}^{2}\left(\mathbb{R}^{4}\right)$ (see $\S 7$ for a review of the representations of $S O(4)$ ). But $\mathbb{R}^{4} \otimes \mathbb{R}^{4} \cong \operatorname{Sym}^{2}\left(\mathbb{R}^{4}\right) \oplus$ $\Lambda^{2}\left(\mathbb{R}^{4}\right)$, and there are no nontrivial equivariant maps from $\Lambda^{2}\left(\mathbb{R}^{4}\right)$ to the irreducible representation $\operatorname{Sym}_{0}^{2}\left(\mathbb{R}^{4}\right)$. Thus each of the maps above must factor through a map from $\mathbb{R}^{4} \otimes \mathbb{R}^{4}$ to $\operatorname{Sym}^{2}\left(\mathbb{R}^{4}\right)$, implying that (2.9) and (2.10) are symmetric.

- Proof of Proposition 2.1. Writing components relative to a local orthonormal basis $\left\{\theta^{a}\right\}$ of $T^{*} M$, we have

$$
\Delta_{0}^{A}\left(\iota_{Z} \alpha\right)=\Delta^{A}\left(\iota_{Z} \alpha\right)=\iota_{\Delta Z} \alpha-2 \iota_{\nabla_{a} Z} \nabla_{a}^{A} \alpha+\iota_{Z} \Delta^{A} \alpha .
$$

The Weitzenböck identity for one-forms (see for example [FU, equation (6.25)]) asserts that

$$
\Delta^{A} \alpha=\Delta_{1}^{A} \alpha+2\left[F^{A}, \alpha\right]-\operatorname{Ric}(\alpha)
$$


where $[F, \alpha]=\left[F_{a b}, \alpha_{b}\right] \otimes \theta^{a}$. But

$$
\iota_{Z}[F, \alpha]=\left[\left(\iota_{Z} F\right)_{b}, \alpha_{b}\right]=\{\tilde{Z}, \alpha\}
$$

so

$$
\Delta_{0}^{A}\left(\frac{1}{2} \iota_{Z} \alpha\right)=\{\tilde{Z}, \alpha\}-\mathrm{R}_{0}(\tilde{Z}, \alpha)
$$

which is equivalent to $(2.2)$.

Moving on to (2.3), we recall Weitzenböck identity for anti-self-dual twoforms ([FU, equation (6.26)]),

$$
\Delta_{A} \eta=2 \Delta_{-}^{A} \eta+\mathcal{R}_{-}(\eta)
$$

Expanding as in (2.11) and using both (2.12) and (2.13), we have

$$
\Delta_{-}^{A}\left(Z^{*} \wedge \alpha\right)_{-}=p_{-}\left(Z^{*} \wedge[F, \alpha]\right)-\mathrm{R}_{2}(\tilde{Z}, \alpha) .
$$

Since

$$
\begin{aligned}
p_{-}\left(Z^{*} \wedge[F, \alpha]\right) & =Z_{a}\left[F_{b c}, \alpha_{c}\right]\left(\theta^{a} \wedge \theta^{b}\right)_{-} \\
& =Z_{a}\left[F_{b a}, \alpha_{c}\right]\left(\theta^{c} \wedge \theta^{b}\right)_{-} \quad(\text { symmetry of }(2.10)) \\
& =\left[\left(i_{Z} F\right)_{b}, \alpha_{c}\right]\left(\theta^{b} \wedge \theta^{c}\right)_{-} \\
& =[\tilde{Z}, \alpha]_{-}
\end{aligned}
$$

we have

$$
\Delta_{-}^{A}\left(Z^{*} \wedge \alpha\right)_{-}=[\tilde{Z}, \alpha]_{-}-\mathrm{R}_{2}(\tilde{Z}, \alpha)
$$

and (2.3) follows.

We begin to examine the implications of all this for the curvature formula (1.3). First, for arbitrary $A \in \mathcal{M}_{\lambda_{0}}$ and $X, Y \in \Omega^{1}($ Ad $P)$, define

$$
\begin{gathered}
\operatorname{num}(X, Y ; A)=\text { right-hand side of }(1.3), \\
\operatorname{denom}(X, Y)=\|X\|_{2}^{2}\|Y\|_{2}^{2}-\langle X, Y\rangle^{2}
\end{gathered}
$$

Thus for arbitrary non-proportional $X, Y \in H_{A}$ the sectional curvature is given by

$$
\sigma_{A}(X, Y)=\frac{\operatorname{num}(X, Y ; A)}{\operatorname{denom}(X, Y ; A)}
$$


We define the approximate sectional curvature $\tilde{\sigma}$ by

$$
\tilde{\sigma}(\tilde{Z}, \tilde{W})=\frac{\operatorname{num}(\tilde{Z}, \tilde{W} ; A)}{\operatorname{denom}\left(\pi_{A} \tilde{Z}, \pi_{A} \tilde{W}\right)}
$$

for (non-proportional) $\tilde{Z}, \tilde{W} \in \tilde{H}_{A}$. Now any 2-plane in $\tilde{H}_{A}$ has a basis of the form

$$
\tilde{Z}=\tilde{Z}_{\mathbf{a}}, \quad \tilde{W}=\tilde{Z}_{\mathbf{b}}+k \tilde{Z}^{\prime}
$$

where

$$
\mathbf{a} \perp \mathbf{b}, \quad|\mathbf{a}|^{2}=|\mathbf{b}|^{2}+2 k^{2} \lambda^{2}=1 .
$$

From [GP2, §3] we know that

$$
\operatorname{denom}\left(\pi_{A} \tilde{Z}, \pi_{A} \tilde{W}\right) \rightarrow(2 \pi)^{4} \text { as } \lambda \rightarrow 0
$$

for such a basis, so we need focus only on $\operatorname{num}(\tilde{Z}, \tilde{W} ; A)$. Using $(2.6)$ in the first term, we have

$$
\begin{aligned}
\langle\{\tilde{Z}, \tilde{W}\}, G\{\tilde{Z}, \tilde{W}\}\rangle & =\left\langle\{\tilde{Z}, \tilde{W}\},-\frac{1}{2} F(Z, W)+G(\mathrm{R})\right\rangle \\
& =-\frac{1}{2}\langle\{\tilde{Z}, \tilde{W}\}, F(Z, W)\rangle+\langle G\{\tilde{Z}, \tilde{W}\}, \mathrm{R}\rangle \\
& =-\frac{1}{2}\langle\{\tilde{Z}, \tilde{W}\}, F(Z, W)\rangle-\frac{1}{2}\langle F(Z, W), \mathrm{R}\rangle+\langle G(\mathrm{R}), \mathrm{R}\rangle .
\end{aligned}
$$

We apply the same argument to the other two terms, after rewriting the last as $\left\langle[\tilde{W}, \tilde{W}]_{-}, G_{-}^{A}[\tilde{Z}, \tilde{Z}]_{-}\right\rangle$. We then have

$$
\operatorname{num}(\tilde{Z}, \tilde{W})=\mathrm{L} . \mathrm{T} .(Z, W)+\operatorname{Rem}(Z, W),
$$

where the leading term L.T. $(Z, W)$ is defined by

$$
\begin{aligned}
\text { L. T. }(Z, W)= & \frac{3}{2}\left\langle\{\tilde{Z}, \tilde{W}\}, \iota_{Z} \tilde{W}\right\rangle \\
& \quad-\left\langle[\tilde{Z}, \tilde{W}]_{-},\left(Z^{*} \wedge \tilde{W}\right)_{-}\right\rangle+\left\langle[\tilde{W}, \tilde{W}]_{-},\left(Z^{*} \wedge \tilde{Z}\right)_{-}\right\rangle .
\end{aligned}
$$

(The remainder term $\operatorname{Rem}(Z, W)$ is written out explicitly in (8.2).) This leading term is the integral of the local expression we have been seeking. Each inner product separately diverges at the same rate as $\int_{M}|F|^{3} \sim \lambda^{-2}$ (see Lemma 3.3), but, as the next lemma shows, the local inner products cancel. 
Lemma 2.4. Let $(\cdot, \cdot)$ denote pointwise inner product. Let $Z$ be any vector field and let $\alpha, \eta$ be any Ad P-valued 1-forms. Then

$$
\frac{3}{2}\left(\{\alpha, \eta\}, \iota_{Z} \eta\right)-\left([\alpha, \eta]_{-},\left(Z^{*} \wedge \eta\right)_{-}\right)+\left([\eta, \eta]_{-},\left(Z^{*} \wedge \alpha\right)_{-}\right) \equiv 0
$$

Proof. Let $\left\{\theta^{a}\right\}$ be a local orthonormal basis of $T^{*} M$. Using Ad-invariance of the inner product on Ad $P$ and a basic property of the $*$-operator, we have

$$
\begin{aligned}
\left([\eta, \eta]_{-}, *\left(Z^{*} \wedge \alpha\right)\right) & =\left(\left[\eta_{a}, \eta_{b}\right], \alpha_{c}\right)\left(\theta^{a} \wedge \theta^{b}, *\left(Z^{*} \wedge \theta^{c}\right)\right) \\
& =\left(\eta_{b},\left[\eta_{a}, \alpha_{c}\right]\right)\left(\theta^{a} \wedge \theta^{c}, *\left(Z^{*} \wedge \theta^{b}\right)\right. \\
& =\left([\alpha, \eta], *\left(Z^{*} \wedge \eta\right)\right) .
\end{aligned}
$$

Thus

$$
\begin{aligned}
& 2\left([\eta, \eta]_{-},\left(Z^{*} \wedge \alpha\right)_{-}\right)-2\left([\alpha, \eta]_{-},\left(Z^{*} \wedge \alpha\right)_{-}\right) \\
= & \left([\eta, \eta], Z^{*} \wedge \alpha\right)-\left([\alpha, \eta], Z^{*} \wedge \eta\right) \\
= & \left(\iota_{Z}[\eta, \eta], \alpha\right)-\left(\iota_{Z}[\alpha, \eta], \eta\right) \\
= & \left(2\left[\iota_{Z} \eta, \eta\right], \alpha\right)-\left(\left[\iota_{Z} \alpha, \eta\right]-\left[\alpha, \iota_{Z} \eta\right], \eta\right) \\
= & 2\left(\iota_{Z} \eta,\{\eta, \alpha\}\right)-\left(\iota_{Z} \alpha,\{\eta, \eta\}\right)-\left(\iota_{Z} \eta,\{\alpha, \eta\}\right) \\
= & -3\left(\{\alpha, \eta\}, \iota_{Z} \eta\right),
\end{aligned}
$$

and (2.23) follows.

Corollary 2.5. The expression L. T. $(Z, W)$ in (2.22) vanishes identically. Hence

$$
\tilde{\sigma}_{A}(\tilde{Z}, \tilde{W})=\frac{\operatorname{Rem}(Z, W)}{\operatorname{denom}\left(\pi_{A} \tilde{Z}, \pi_{A} \tilde{W}\right)} .
$$

Proof. Apply the lemma above with $\alpha=\tilde{Z}, \eta=\tilde{W}$.

To complete our localization analysis, we must study the remainder

$$
\operatorname{Rem}(Z, W)
$$

as $\lambda \rightarrow 0$. We defer this until $\S 8$, where we will also consider the error $\sigma-\tilde{\sigma}$ introduced by localization. In the intervening sections we provide the basic estimates we will need. 


\section{An Estimate tOOL-KIT}

From now on we use the symbol $\mathcal{R}(\cdot)$, with zero or more arguments, to denote quantities which depend in some universal and multilinear way on the Riemann tensor (and possibly its derivatives) and the arguments. We continue to use $\left\{\theta^{a}\right\}$ to denote a local orthonormal basis of $T^{*} M$.

We begin with a completely general Weitzenböck-type identity concerning the commutator of a covariant derivative and a Laplacian.

Lemma 3.1. Let $E$ be a vector bundle over a Riemannian manifold $(M, g)$. Let $E$ have a connection $A$ with curvature two-form $K$ and give $T^{*} M$ the Levi-Civita connection. Then for any $s \in \Gamma(E)$,

$$
\nabla^{A}\left(\nabla^{A^{*}} \nabla^{A} s\right)-\nabla^{A^{*}} \nabla^{A}\left(\nabla^{A} s\right)=\operatorname{Ric}\left(\nabla^{A} s\right)+2 K\left(\nabla^{A} s\right)-\left(\nabla^{A^{*}} K\right)(s),
$$

where

$$
\begin{aligned}
\operatorname{Ric}\left(\nabla^{A} s\right) & =\left(R_{a b} \nabla_{a}^{A} s\right) \otimes \theta^{b}, \\
K(\nabla s) & =\left(K_{a b}\left(\nabla_{a}^{A} s\right)\right) \otimes \theta^{b},
\end{aligned}
$$

and

$$
\left(\nabla^{A^{*}} K\right)(s)=-\left(\nabla^{A} K_{a b}\right)(s) \theta^{b}
$$

(In the last formula it is assumed that $d \theta^{a}=0$ at the point at which the right-hand side is evaluated.)

Proof. Fix $q \in M$ and choose $\left\{\theta^{a}\right\}$ so that $d \theta^{a}(q)=0$. Using $\nabla^{A^{*}} \nabla^{A} s=$ $-\operatorname{tr}_{g} \nabla^{A} \nabla^{A} s$, the result follows from a straightforward computation.

This tool, together with the Weitzenböck identity (3.6) and integration by parts, enables us to bound $L^{2}$-norms of arbitrary derivatives of a curvature two-form $F^{A}$, weighted by arbitrary powers of $r$, in terms of weighted norms of $F^{A}$ itself. For connections in $\mathcal{M}_{\lambda_{0}}$, these norms can in turn be bounded in terms of $\lambda$-our goal - thanks to a basic pointwise bound on $\left|F^{A}\right|$. In[GP2], the authors relied on Donaldson's bound

$$
\left|F^{A}\right| \leq c \lambda^{2-\delta} / r^{4-\delta}
$$


where $\delta$ can be made arbitrarily small by taking $r_{0}$ sufficiently small. However, under the $\Lambda_{-}^{2}$ curvature condition, we can eliminate the $\delta$ from this formula and sharpen the estimates.

Theorem 3.2. There exist $r_{0}, \lambda_{0}$, and $c$, independent of $A$, such that if $\lambda(A) \leq$ $\lambda_{0}$ and $(M, g)$ satisfies the $\Lambda_{-}^{2}$ curvature condition at the center point $p$ of $A$, then

$$
\left|F^{A}\right| \leq c \lambda^{2} / r^{4}
$$

on $B_{2 r_{0}}(p)$.

We defer the proof of this estimate to $\S 4$. For the rest of this section, we assume that $r_{0}$ and $\lambda_{0}$ have been chosen small enough so that (3.2) applies; or, when the $\bigwedge_{-}^{2}$ curvature condition is satisfied at $p$, so that (3.3) applies.

Lemma 3.3. Assume the $\bigwedge_{-}^{2}$ curvature condition holds at $p$. For $A \in \mathcal{M}_{\lambda_{0}}$, $k>-2$ and $q \geq 0$, define

$$
I_{k, q}(A)=\int_{B_{2 r_{\varphi}}(p)} r^{2 k}\left|F^{A}\right|^{q} \Omega(g),
$$

where $\Omega(g)$ is the volume form of the metric $g$.

(a) Assume $k>-2$ and let $m$ be arbitrary. Suppose that $m \geq \max (q-$ $k, 2-q)$; if $k=2(q-1)$, assume the strict inequality $m>2-q$. Then there is a constant $c_{m, k, q}$ such that

$$
I_{k, q}(A) \leq c_{m, k, q} \lambda^{4-2 m}
$$

If $k=2(q-1)$ and $m=2-q$, there is a constant $c_{m}$ such that

$$
I_{k, q}(A)=I_{2-2 m, 2-m}(A) \leq c_{m} \lambda^{4-2 m}|\log \lambda| \text {. }
$$

(b) Let $j \geq 0$ and assume $k-j>-2$. If $m>0$ and $k-j \geq 2-m$, or if $m=0$ and $k-j>2$, then there is a constant $c_{j, k, m}$ such that

$$
\left\|\beta r^{k}\left(\nabla^{A}\right)^{j} F^{A}\right\|_{2} \leq c_{j, k, m} \lambda^{2-m} .
$$

In the borderline case $m=0, k=j+2$, there is a constant $c_{j}$ such that

$$
\left\|\beta r^{j+2}\left(\nabla^{A}\right)^{j} F^{A}\right\|_{2} \leq c_{j} \lambda^{2} \sqrt{|\log \lambda|} .
$$


Proof. (a) We break the region of integration into two pieces: the ball $B_{\lambda}$ of radius $\lambda$ and its complement $B_{\lambda}^{\prime}$. There is a constant $c$ such that for $r \leq \lambda$,

$$
|F| \leq c \frac{\lambda^{2}}{\left(r^{2}+\lambda^{2}\right)^{2}}
$$

cf. the proof of Lemma 3.5 in [GP2], for example. Hence

$$
\begin{aligned}
\int_{r \leq \lambda} r^{2 k}|F|^{q} \Omega(g) & \leq c \int_{0}^{\lambda} r^{2 k} \frac{\lambda^{2 q}}{\left(r^{2}+\lambda^{2}\right)^{2 q}} r^{3} d r \\
& \leq c \lambda^{2 k+4-2 q},
\end{aligned}
$$

as we see by substituting $r=\lambda \rho$. If $k \geq q-m$, this will be $\leq c \lambda^{4-2 m}$. On the outside region, using (3.3), we have

$$
\int_{\lambda \leq r \leq 2 r_{0}} r^{2 k}|F|^{q} \Omega(g) \leq c \lambda^{2 q} \int_{\lambda}^{2 r_{0}} r^{2 k+3-4 q} d r .
$$

Let $n=2 k+4-4 q$. If $n>0$ (i.e. $k>2(q-1)$ ), the right-hand side of (3.5) is $\leq c \lambda^{2 q}$; if $n=0$ (i.e. $k=2(q-1)$ ), the right-hand side is $\leq c \lambda^{2 q}|\log \lambda|$; and if $n<0$ (i.e. $k<2(q-1)$ ), the right-hand side is $\leq c \lambda^{2 k+4-2 q}$. In the first two cases, the inequalities $q \geq 2-m$ and $q>2-m$, respectively, ensure a bound of the form $c \lambda^{4-2 m}$, and the requirement $k \geq q-m$ from the inner integral is redundant. In the third case, the stated hypotheses ensure that both the inner and outer integrals have the desired bounds.

(b) We prove only the first inequality; the second is proven identically, up to the appropriate insertions of $\log \lambda$. For $j=0$ (3.4) is an immediate consequence of (a). For $j=1$, we integrate by parts, finding

$$
\begin{aligned}
\left\langle\beta r^{k} \nabla F, \beta r^{k} \nabla F\right\rangle & =\left\langle\nabla^{*}\left(\beta^{2} r^{2 k} \nabla F\right), F\right\rangle \\
& \leq c\left\langle\beta r^{k} \nabla F,(\beta+|d \beta|) r^{k-1} F\right\rangle+\left\langle\beta^{2} r^{2 k} \nabla^{*} \nabla F, F\right\rangle .
\end{aligned}
$$

But the Weitzenböck identity for self-dual two-forms (see [FU, equation (6.26)]) implies that

$$
\Delta^{A} F=-[F, F]+\mathcal{R}(F),
$$

where $[F, F]=\left[F_{a c}, F_{b c}\right] \otimes \theta^{a} \wedge \theta^{b}$. Thus

$$
\begin{aligned}
\left\langle\beta r^{k} \nabla F, \beta r^{k} \nabla F\right\rangle & \leq c\left(\left\|\beta r^{k} \nabla F\right\|_{2}\left(I_{(k-1), 2}\right)^{1 / 2}+I_{k, 2}+I_{k, 3}\right) \\
& \leq \frac{1}{2}\left\|\beta r^{k} \nabla F\right\|_{2}^{2}+c\left(I_{(k-1), 2}+I_{k, 2}+I_{k, 3}\right) .
\end{aligned}
$$


The result for $j=1$ now follows from part (a).

For higher $j$, we proceed by induction. Suppose the result has been established up to some particular $j \geq 1$; we will establish it for $j+1$. In the following, we will occasionally use (3.4) with $\beta$ replaced by other functions $\tilde{\beta}$-e.g. $\tilde{\beta}=\beta+|d \beta|$ or $\tilde{\beta}=\beta^{1 / 2}$-which are supported in $B_{2 r_{0}}$ and have a uniform pointwise bound; it is clear from the proof that (3.4) is equally valid in these cases.

Again we start by integrating by parts as above. Using Lemma 3.1, and simplifying the calculation by noting that since $A$ is Yang-Mills, the term $\nabla^{*} K$ in (3.1) is of the form $\mathcal{R}$ (though the result still holds without this simplification), we find

$$
\begin{aligned}
\left\|\beta r^{k} \nabla^{j+1} F\right\|_{2}^{2} \leq & c\left\|\beta r^{k} \nabla^{j+1} F\right\|_{2}\left\|\tilde{\beta} r^{k-1} \nabla^{j} F\right\|_{2}+\left\langle\nabla\left(\nabla^{*} \nabla\right) \nabla^{j-1} F, \beta^{2} r^{2 k} \nabla^{j} F\right\rangle \\
& +c \int_{M} \beta^{2} r^{2 k}\left(\left|\nabla^{j} F\right|^{2}+\left|F\left\|\left.\nabla^{j} F\right|^{2}+\left|\nabla^{j} F \| \nabla^{j-1} F\right|\right) \Omega(g) .\right.\right.
\end{aligned}
$$

Integrating by parts one more time, and noting that $\left|\nabla^{*} \nabla^{j} F\right| \leq\left|\nabla^{j+1} F\right|$, we find that the first two terms on the right-hand side are together bounded by

$$
c\left\|\beta r^{k} \nabla \nabla F\right\|_{2}\left(\left\|\tilde{\beta} r^{k-1} \nabla^{j} F\right\|_{2}+\left\|\beta r^{k}\left(\nabla^{*} \nabla\right) \nabla^{j-1} F\right\|_{2}\right) .
$$

The middle term in the integral in (3.7) gives a contribution bounded by

$$
\left\|\beta r^{k} \nabla^{j} F\right\|_{4}\left\|\beta r^{k-1} \nabla^{j} F\right\|_{2}\|r F\|_{L^{4}\left(B_{2 r_{0}}\right)} .
$$

By part (a) with $m=2$ we have $\|r F\|_{L^{4}\left(B_{2 r_{0}}\right)} \leq c$, and the four-dimensional Sobolev embedding $L_{1}^{2} \hookrightarrow L^{4}$, supplemented by Kato's inequality

$$
|d| s|| \leq|\nabla s|
$$

implies

$$
\begin{aligned}
\left\|\beta r^{k} \nabla^{j} F\right\|_{4} & \leq c\left(\left\|\beta r^{k} \nabla^{j} F\right\|_{2}+\left\|\nabla\left(\beta r^{k} \nabla^{j} F\right)\right\|_{2}\right) \\
& \leq c\left(\left\|\tilde{\beta} r^{k-1} \nabla^{j} F\right\|_{2}+\left\|\beta r^{k} \nabla^{j+1} F\right\|_{2}\right) .
\end{aligned}
$$

Absorbing the first term into (3.8), we then find that the right-hand side of (3.7) is bounded by

$$
\text { expression }(3.8)+c\left(\left\|\tilde{\beta} r^{k-1} \nabla^{j} F\right\|_{2}^{2}+\left\|\beta r^{k} \nabla^{j} F\right\|_{2}\left\|\beta r^{k} \nabla^{j-1} F\right\|_{2}\right) \text {. }
$$


Using the inductive hypothesis, it is easy to check that the terms in parentheses are bounded by $c \lambda^{4-2 m}$. Combining (3.7), (3.8), and (3.11), we therefore have

$$
\left\|\beta r^{k} \nabla^{j+1} F\right\|_{2}^{2} \leq c\left(\left\|\beta r^{k}\left(\nabla^{*} \nabla\right) \nabla^{j-1} F\right\|_{2}^{2}+\lambda^{4-2 m}\right)
$$

so we will be done if we can show that

$$
\left\|\beta r^{k}\left(\nabla^{*} \nabla\right) \nabla^{j-1} F\right\|_{2} \leq c \lambda^{2-m}
$$

To establish (3.12), we use Lemma (3.1) inductively and the Weitzenböck formula (3.6) to show

$$
\nabla^{*} \nabla\left(\nabla^{j-1} F\right)=\sum_{i=0}^{j-1} \mathcal{R}\left(\nabla^{i} F\right)+\sum_{i=0}^{[(j-1) / 2]} c_{i}\left[\nabla^{i} F, \nabla^{j-1-i} F\right]
$$

Hence

$$
\begin{aligned}
\left\|\beta r^{k} \nabla^{*} \nabla\left(\nabla^{j-1} F\right)\right\|_{2} \leq c\left(\sum_{i=0}^{j-1}\left\|\beta r^{k} \nabla^{i} F\right\|_{2}\right. & \\
& \left.+\sum_{i=0}^{[(j-1) / 2]}\left\|\tilde{\beta} r^{i+1} \nabla^{i} F\right\|_{4}\left\|\tilde{\beta} r^{k-i-1} \nabla^{j-i-1} F\right\|_{4}\right) .
\end{aligned}
$$

In the first sum, the inductive hypothesis implies that every term is bounded by $c \lambda^{2-m}$. In the second, we apply (3.10) to each term, bounding the $i$ 'th term by

$$
c\left(\left\|\tilde{\beta} r^{i} \nabla^{i} F\right\|_{2}+\left\|\tilde{\beta} r^{i+1} \nabla^{i+1} F\right\|_{2}\right)\left(\left\|\tilde{\beta} r^{k-i-2} \nabla^{j-1-i} F\right\|_{2}+\left\|\tilde{\beta} r^{k-i-1} \nabla^{j-i} F\right\|_{2}\right) .
$$

Using the inductive hypothesis with $m=2$, the first two $L^{2}$-norms above are $\leq c$, while the last two are $\leq c \lambda^{2-m}$. This establishes (3.12), and the proof is complete.

The bounds of greatest use to us are summarized in the following corollary. 
Corollary 3.4. Assume the $\bigwedge_{-}^{2}$ curvature condition is satisfied at $p$. For $A \in \mathcal{M}_{\lambda_{0}}, k>-2$, and $j \geq 0$, the following bounds hold.

$$
\begin{gathered}
\left\|\beta r^{k} F\right\|_{1} \leq c \begin{cases}\lambda^{2} & \text { if } k>0 \\
\lambda^{2}|\log \lambda| & \text { if } k=0\end{cases} \\
\left\|\beta r^{k+j} \nabla^{j} F\right\|_{2} \leq c \begin{cases}\lambda^{k} & \text { if } k<2 \\
\lambda^{2} \sqrt{|\log \lambda|} & \text { if } k=2 . \\
\lambda^{2} & \text { if } k>2\end{cases} \\
\left\|\beta r^{k+j} \nabla^{j} F\right\|_{4} \leq c \begin{cases}\lambda^{k-1} & \text { if } k<3 \\
\lambda^{2} \sqrt{|\log \lambda|} & \text { if } k=3 . \\
\lambda^{2} & \text { if } k>3\end{cases}
\end{gathered}
$$

Proof. The $L^{1}$-bounds are immediate consequences of part (a) of the lemma, while the $L^{2}$-bounds follow from part (b). For the $L^{4}$-bounds we use (3.10) and the $L^{2}$-bounds.

Remark 3.1. In the absence of the $\bigwedge_{-}^{2}$ curvature condition we would of course get weaker estimates in certain cases. All appearances of $\lambda^{2}$ in (3.13-3.15) would be replaced by $\lambda^{2-\delta_{1}}$ (and we could of course erase the logs), and the restrictions $k<2$ and $k<3$ in (3.14) and (3.15) would be replaced by $k<2-\delta_{2}$ and $k<3-\delta_{3}$, respectively. Here the $\delta_{i}$ are proportional to the $\delta$ in (3.2).

We conclude this section with a very useful and general Sobolev-type inequality, and an application to instantons in $\mathcal{M}_{\lambda_{0}}$.

Lemma 3.5. Let $E,(M, g)$ and $A$ be as in Lemma 3.1, with $M$ compact and $n=\operatorname{dim}(M)>2$. Let $r_{1}$ be the injectivity radius of $(M, g)$. There exists a constant $c$, independent of $A$, such that for any $p \in M$ and any section $s$ of E supported in $B_{r_{1}}(p)$,

$$
\left\|r^{-1} s\right\|_{2} \leq c\left\|\nabla^{A} s\right\|_{2}
$$

where $r=\operatorname{dist}(p, \cdot)$. Without any assumption on the support of $s$, an inequality of the form

$$
\left\|r^{-1} s\right\|_{2} \leq c\left(\left\|\nabla^{A} s\right\|_{2}+\|s\|_{2}\right)
$$

holds. 
There is nothing special about the $L^{2}$ norm in this lemma; as the proof below shows, the same inequality holds with $L^{p}$ norms on an $n$-dimensional manifold as long as $p<n$.

Proof. Since $M$ is compact, the $L^{2}$ norm over $B_{r_{1}}(p)$ determined by $g$ is bounded above and below, uniformly in $p$, by positive multiples of the $L^{2}$ norm determined by the flat metric given by a normal coordinate system. Hence it suffices to prove (3.16) on $\mathbb{R}^{n}$, where we let $r$ denote distance to the origin. For $f \in C_{0}^{\infty}\left(\mathbb{R}^{n}\right)$ we always have $f / r \in L^{2}$. Expressing $\|f / r\|_{2}^{2}$ as an integral in polar coordinates and integrating with respect to $r$ by parts, one finds

$$
\|f / r\|_{2} \leq c(n)\|d f\|_{2} .
$$

Letting $f=|s|$, where $s$ is a compactly supported section of a vector bundle over $\mathbb{R}^{n}$ with connection $\nabla$, Kato's inequality (3.9) then gives (3.16). To obtain (3.17) we simply note that

$$
\left\|r^{-1} s\right\|_{2} \leq\left\|\beta r^{-1} s\right\|_{2}+\left\|(1-\beta) r^{-1} s\right\|_{2} \leq\left\|\beta r^{-1} s\right\|_{2}+c\|s\|_{2}
$$

and apply (3.16).

This lemma has a strong implication for collar instantons.

Corollary 3.6. There exist constants $c$ such that for $A \in \mathcal{M}_{\lambda_{0}}$ and $\eta, \eta^{\prime} \in$ $\Omega_{-}^{2}($ Ad $P)$,

$$
\begin{gathered}
\left\|r^{-1} \eta\right\|_{2} \leq c\left\|\left(d_{A}^{-}\right)^{*} \eta\right\|_{2} \leq c\left\|\nabla^{A} \eta\right\|_{2} \\
\left\|r^{-1} G_{-}^{A}(\eta)\right\|_{2} \leq c\|r \eta\|_{2}
\end{gathered}
$$

and

$$
\left|\left\langle\eta, G_{-}^{A} \eta^{\prime}\right\rangle\right| \leq c\|r \eta\|_{2}\left\|r \eta^{\prime}\right\|_{2} .
$$

Proof. Recall that the first eigenvalue of $\Delta_{-}^{A}$ is bounded away from 0 on $\mathcal{M}_{\lambda_{0}}$ : for $\eta \in \Omega_{-}^{2}(\operatorname{Ad} P)$,

$$
\|\eta\|_{2} \leq c\left\|\Delta_{-}^{A} \eta\right\|_{2}
$$

or equivalently

$$
\|\eta\|_{2} \leq c\left\|\left(d_{A}^{-}\right)^{*} \eta\right\|_{2}
$$


(This is a consequence of the positive-definiteness of $M$; see [L, Ch. VI §3].) Thus, using the Weitzenböck formula (2.13) we have

$$
\begin{aligned}
\left\|\nabla^{A} \eta\right\|_{2}^{2} & =\left\langle\Delta^{A} \eta, \eta\right\rangle \\
& =\left\langle 2 \Delta_{-}^{A} \eta+\mathcal{R}(\eta), \eta\right\rangle \\
& \leq c\left(\left\|\left(d_{A}^{-}\right)^{*} \eta\right\|_{2}^{2}+\|\eta\|_{2}^{2}\right) \\
& \leq c\left\|\left(d_{A}^{-}\right)^{*} \eta\right\|_{2}^{2}
\end{aligned}
$$

so

$$
\left\|\nabla^{A} \eta\right\|_{2} \leq c\left\|\left(d_{A}^{-}\right)^{*} \eta\right\|_{2} \leq c\left\|\nabla^{A} \eta\right\|_{2} .
$$

Combining Lemma 3.5 with (3.21) and (3.22) we obtain (3.18). Using the same tools, we have

$$
\begin{aligned}
\left\|\left(d_{A}^{-}\right)^{*} G_{-}^{A} \eta\right\|_{2}^{2} & =c\left\langle r^{-1} G_{-}^{A} \eta, r \eta\right\rangle \\
& \leq c\left\|\left(d_{A}^{-}\right)^{*} G_{-}^{A} \eta\right\|_{2}\|r \eta\|_{2},
\end{aligned}
$$

implying

$$
\left\|\left(d_{A}^{-}\right)^{*} G_{-}^{A} \eta\right\|_{2} \leq c\|r \eta\|_{2} .
$$

Using (3.18) a final time, we arrive at (3.19). Equation (3.20) follows immediately since $\left\langle\eta, G_{-}^{A} \eta^{\prime}\right\rangle=\left\langle r \eta, r^{-1} G_{-}^{A} \eta^{\prime}\right\rangle$.

\section{The DecaY estimate}

To prove Theorem 3.2 we must first review Donaldson's original estimate ([D1, Theorem 16(ii)] ; see also [DK, §7.3]). We concentrate on the formal aspects of the argument, carefully keeping track of a sign (see (4.13)) that was irrelevant in the presentations above. For the analytic details not discussed here, we refer the reader to [DK].

Below, $\epsilon$ is an adjustable small constant that we keep taking smaller as needed, updating the values of $\lambda_{0}$ and $r_{0}$ accordingly. Throughout this section $\left\{x^{i}\right\}$ is a fixed normal coordinate system centered at $p$, ordered consistently with the orientation of $M$.

Let $B$ be the disk $B_{3 r_{0}}(p)$. Using rescaled normal coordinates $y^{i}=x^{i} / \lambda$, we identify $B$ with the ball $B^{\prime}=B_{3 r_{0} / \lambda}(0)$ in $\mathbb{R}^{4}$, and give $B^{\prime}$ a metric $g_{\lambda}$ equal 
to $\lambda^{-2}$ times the metric pulled back from $B$. Note that (3.3) is equivalent to

$$
\left|F^{A}\right|_{g_{\lambda}} \leq c \rho^{-4} \text { on } B^{\prime}
$$

where $\rho^{2}=\sum\left(y^{i}\right)^{2}$. We recall that as $\lambda \rightarrow 0$, an instanton $A$ of scale $\lambda$, in the coordinates $\left\{y^{i}\right\}$ and metric $g_{\lambda}$, is closely approximated by the standard instanton on $\mathbb{R}^{4}$ on any compact subset of $B^{\prime}$ ([D1, Theorem 16(i)]; see also [GP2, §3, 'Fact A']). Thus, given $N$ there exists $\lambda_{0}$ such that if $\lambda<\lambda_{0}$ then $\left|F^{A}\right|_{g_{\lambda}}<c\left(1+\rho^{2}\right)^{-2}$ on $B_{N}(0)$,so we need only consider the complement of $B_{N}(0)$.

Given $\epsilon$, the approximability of $A$ by the standard instanton lets us first choose $N=N(\epsilon)$ (independent of $p$ ) and then $\lambda_{0}=\lambda_{0}(\epsilon)$ so that

$$
8 \pi^{2}-\epsilon<\int_{B_{N-1}(0)}\left|F^{A}\right|_{g_{\lambda}}^{2} \Omega\left(g_{\lambda}\right) \leq 8 \pi^{2}
$$

for all $A \in \mathcal{M}_{\lambda_{0}}$. (Here and below $\Omega(h)$ denotes the Riemannian volume density of a metric $h$.)

On $B^{\prime}-\{0\}$ we can write $g_{\lambda}=d \rho^{2} \oplus \rho^{2} \hat{g_{\rho}}$ for a family of metrics $\left\{g_{\rho}\right\}$ on the sphere $\left\{\sum\left(y^{i}\right)^{2}=1\right\}$; the sum is orthogonal by the Gauss lemma. Writing $\rho=N e^{t}$, we can re-express this as

$$
g_{\lambda}=N^{2} e^{2 t} \tilde{g}
$$

where

$$
\tilde{g}=d t^{2} \oplus \hat{g}_{t}
$$

and $\hat{g}_{t}$ is a metric on $\mathbb{S}^{3}$. We write $\hat{g}_{s t d}$ for the standard metric on the sphere. Tracing through the conformal changes, letting $u^{i}=y^{i} / \rho$, and using the Taylor expansion of the original metric $g$ at $p$, we have

$$
\hat{g}_{t}=\hat{g}_{s t d}-\frac{1}{3} \lambda^{2} N^{2} e^{2 t} R_{i k j l}(p) u^{k} u^{l} d u^{i} \otimes d u^{j}+O\left(\left(N \lambda e^{t}\right)^{3}\right)
$$

where $R_{i j k l}$ are the components of the Riemann tensor of $g$ with respect to $\left\{x^{i}\right\}$. Since $\lambda N e^{t} \leq 3 r_{0}$, by taking $r_{0}$ small enough from the start we can ensure that $\hat{g}_{t}$ is in a given $C^{0}$-neighborhood of $\hat{g}_{s t d}$ for all $p, \lambda, t$.

Using (4.3) to rewrite (4.1) what we now need to show is

$$
\left|F^{A}\right|_{\tilde{g}} \leq c e^{-2 t} \quad \text { on } \quad\left[0, \log \left(2 r_{0} / \lambda\right)\right] \times \mathbb{S}^{3} .
$$


Let $T+1=\log \left(3 r_{0} / \lambda\right)$. For $0 \leq t \leq T+1$ we define

$$
E(t)=\int_{[t, T+1] \times \mathbb{S}^{3}}\left|F^{A}\right|_{\tilde{g}}^{2} \Omega(\tilde{g})
$$

Because of $(4.2)$,

$$
0 \leq E(t) \leq \epsilon \quad \text { for } \quad t \in[0, T+1]
$$

(since the $L^{2}$ norm on 2-forms is conformally invariant). As explained in [DK], by taking $\epsilon$ sufficiently small (and $r_{0}$ as well, so that each $\hat{g}_{t}$ is close enough to $\hat{g}_{s t d}$ ) we can ensure each of the following.

$$
\sup _{[1, T+1] \times \mathbb{S}^{3}}\left\{\left|F^{A}\right|(t, u)\right\} \leq c \epsilon .
$$

- Let $j_{t}$ denote the inclusion $\mathbb{S}^{3} \hookrightarrow\{t\} \times \mathbb{S}^{3} \hookrightarrow[0, T+1] \times \mathbb{S}^{3}$ and let $A_{t}$ be the restricted connection $j_{t}^{*} A$. For each $t \in[1, T+1]$ there exists a gauge $\gamma_{t}$-a section of $P$ over $\{t\} \times \mathbb{S}^{3}$ - such that if $a_{t}$ is the connection form of $A_{t}$ in this gauge, then

$(4.7)\left\|j_{t}^{*} a_{t}\right\|_{L_{2}^{1}\left(\mathbb{S}^{3}\right)}+\left\|j_{t}^{*} a_{t}\right\|_{L^{3}\left(\mathbb{S}^{3}\right)}+\left\|j_{t}^{*} a_{t}\right\|_{L^{4}\left(\mathbb{S}^{3}\right)} \leq c\left\|F^{A_{t}}\right\|_{L^{2}\left(\mathbb{S}^{3}\right)} \leq c \epsilon$, where all norms are taken with respect to $\hat{g}_{t}$.

- For $(t, u) \in[0, T] \times \mathbb{S}^{3}$,

$$
\left|F^{A}\right|_{\tilde{g}}^{2}(u, t) \leq c \int_{[t-1, t+1] \times \mathbb{S}^{3}}\left|F^{A}\right|_{\tilde{g}}^{2} \Omega(\tilde{g})
$$

As in [D1] we obtain from the estimates above a differential inequality involving Chern-Simons 3-forms $C S\left(a_{t}\right)$. These are defined for $\mathfrak{s u}(2)$-valued 1-forms $a$ by

$$
C S(a)=\operatorname{tr}\left(a \wedge d a+\frac{2}{3} a \wedge a \wedge a\right)
$$

here " $t r$ " denotes trace in the standard representation. If $a$ is a connection form in some gauge for a connection $A^{\prime}$ then

$$
d(C S(a))=\operatorname{tr}\left(F^{A^{\prime}} \wedge F^{A^{\prime}}\right) .
$$

In addition, if $a, a^{\prime}$ are connection forms in two different gauges for the same connection on a bundle over a closed oriented three-manifold $Y$, then

$$
\int_{Y} C S(a)-\int_{Y} C S\left(a^{\prime}\right) \in 8 \pi^{2} \mathbb{Z}
$$


We can express $E(t)$ in terms of the Chern-Simons forms. First, since the cylinder retracts to $\mathbb{S}^{3}$ we can extend each gauge $\gamma_{t}$ over the whole cylinder. Since $F^{A}$ is self-dual we then have

$$
\begin{aligned}
E(t) & =\int_{[t, T+1] \times \mathbb{S}^{3}}-\operatorname{tr}\left(F^{A} \wedge * F^{A}\right) \\
& =-\int_{[t, T+1] \times \mathbb{S}^{3}} d\left(C S\left(a_{T+1}\right)\right) \\
& =\int_{\{t\} \times \mathbb{S}^{3}} C S\left(a_{T+1}\right)-\int_{\{T+1\} \times \mathbb{S}^{3}} C S\left(a_{T+1}\right) .
\end{aligned}
$$

If we take $\epsilon$ small enough, then in the integral over $\{t\} \times \mathbb{S}^{3}$ we can actually replace $a_{T+1}$ by $a_{t}$. To see this we note that

$$
\begin{aligned}
\mid \int_{\{t\} \times \mathbb{S}^{3}} C S\left(a_{T+1}\right) & -\int_{\{t\} \times \mathbb{S}^{3}} C S\left(a_{t}\right) \mid \\
& \leq E(t)+\left|\int_{\{t\} \times \mathbb{S}^{3}} C S\left(a_{t}\right)\right|+\left|\int_{\{T+1\} \times \mathbb{S}^{3}} C S\left(a_{T+1}\right)\right| \leq c \epsilon
\end{aligned}
$$

by (4.6-4.7). But the two integrals on the left-hand side are congruent mod $8 \pi^{2} \mathbb{Z}$; hence, if $\epsilon$ is small enough, they must be equal. Thus

$$
E(t)=\int_{\mathbb{S}^{3}} C S\left(j_{t}^{*} a_{t}\right)-\int_{\mathbb{S}^{3}} C S\left(j_{T+1}^{*} a_{T+1}\right) .
$$

Furthermore each integral in (4.9) is positive, for if $a_{B}$ is the connection form for $A$ in some gauge defined over the original ball $B$, then using (4.2) we have

$$
\begin{aligned}
8 \pi^{2}-\epsilon \leq \int_{B_{\lambda N e^{t}}(p)}\left|F^{A}\right|_{g}^{2} \Omega(g) \\
=-\int_{B_{\lambda N e^{t}}(p)} d\left(C S\left(a_{B}\right)\right)=-\int_{\mathbb{S}^{3}} C S\left(j_{t}^{*} A_{B}\right) \leq 8 \pi^{2} .
\end{aligned}
$$

But, mod $8 \pi^{2}$, this last integral is congruent to $\int_{\mathbb{S}^{3}} C S\left(j_{t}^{*} a_{t}\right)$, whose absolute value is bounded by $c \epsilon$. Thus $-\int_{\mathbb{S}^{3}} C S\left(j_{t}^{*} a_{t}\right)$ is a small negative number, and our assertion follows. Consequently, if we define

$$
J(t)=\int_{\mathbb{S}^{3}} C S\left(j_{t}^{*} a_{t}\right)
$$

then

$$
0 \leq E(t)=J(t)-J(T+1) \leq J(t) \leq c \epsilon
$$


Because $F^{A}$ is self-dual and the decomposition (4.4) is orthogonal, we have

$$
\left|F^{A}\right|_{\tilde{g}}^{2}(t, u)=2\left|F^{A_{t}}\right|_{\hat{g}_{t}}^{2}(u)
$$

for all $(t, u)$ in our cylinder. Hence differentiating the equality in (4.10) gives

$$
\frac{d J}{d t}=\frac{d E}{d t}=-\int_{\{t\} \times \mathbb{S}^{3}}\left|F^{A}\right|_{\hat{g}_{t}}^{2} \Omega\left(\hat{g}_{t}\right)
$$

$$
=-2 \int_{\mathbb{S}^{3}}\left|F^{A_{t}}\right|_{\hat{g}_{t}}^{2} \Omega\left(\hat{g}_{t}\right)
$$

$$
\leq-2 \int_{\mathbb{S}^{3}}\left|d a_{t}\right|_{\hat{g}_{t}}^{2} \Omega\left(\hat{g}_{t}\right)+c\left\|a_{t}\right\|_{L^{4}\left(\mathbb{S}^{3} ; \hat{g}_{t}\right)}^{2}\left(\left\|a_{t}\right\|_{L_{1}^{2}\left(\mathbb{S}^{3} ; \hat{g}_{t}\right)}^{2}+\left\|a_{t}\right\|_{L^{4}\left(\mathbb{S}^{3} ; \hat{g}_{t}\right)}^{2}\right),
$$

since $\left|F^{A_{t}}\right|=\left|d a_{t}+a_{t} \wedge a_{t}\right|$.

Given a metric $h$ on $\mathbb{S}^{3}$, let $\mu(h)$ be the largest number such that, for any real-valued 1-form $\omega$,

$$
-\int_{\mathbb{S}^{3}} \omega \wedge d \omega \leq \frac{1}{\mu(h)} \int_{\mathbb{S}^{3}}|d \omega|_{h}^{2} \Omega(h) .
$$

It follows from elliptic theory that $\mu(h)>0$; in fact $\mu(h)$ can be characterized as the smallest positive eigenvalue of the operator

$$
D_{h}=-*_{h} d
$$

on the $h$-co-closed 1 -forms on $\mathbb{S}^{3}$. (Here of course $*_{h}$ is the Hodge star operator for the metric $h$. In addition, the restriction to co-closed forms is unnecessary; see below.) It is the sign in (4.13) that we must keep straight to understand the $\bigwedge_{-}^{2}$ curvature condition.

Let us write $\mu_{t}=\mu\left(\hat{g}_{t}\right)$. If we write $a_{t}=a_{t}^{\alpha} e_{\alpha}$, where $\left\{e_{\alpha}\right\}$ is an orthonormal basis of $\mathfrak{s u}(2)$ (so $\operatorname{tr}\left(e_{\alpha} e_{\beta}\right)=-\delta_{\alpha \beta}$ ), we then have

$$
\begin{aligned}
\int_{\mathbb{S}^{3}}\left|d a_{t}\right|_{\hat{g}_{t}}^{2} \Omega\left(\hat{g}_{t}\right) & =\int_{\mathbb{S}^{3}} \sum\left|d a_{t}^{\alpha}\right|_{\hat{g}_{t}}^{2} \Omega\left(\hat{g}_{t}\right) \\
& \geq-\mu_{t} \int_{\mathbb{S}^{3}} \sum a_{t}^{\alpha} \wedge d a_{t}^{\alpha} \\
& =\mu_{t} \int_{\mathbb{S}^{3}} \operatorname{tr}\left(a_{t} \wedge d a_{t}\right) \\
& \geq \mu_{t}\left(J(t)-c\left\|j_{t}^{*} a_{t}\right\|_{L^{3}\left(\mathbb{S}^{3} ; \hat{g}_{t}\right)}^{3}\right) .
\end{aligned}
$$


The eigenvalue $\mu_{t}$ is continuous in $t$, so from (4.7), (4.12), (4.14), and (4.11) we have

$$
\frac{d J}{d t} \leq-2 \mu_{t} J+c\left|\frac{d J}{d t}\right|^{3 / 2}
$$

Let us set

$$
\mu_{\infty}=\inf \left\{\mu_{t}\right\},
$$

where the infimum is taken not just over $t \in[0, T]$ but over the center point $p$ of the construction as well. Because $|J|$ and $|d J / d t|$ are small, the differential inequality above can then be integrated to yield $J(t) \leq c e^{-2 \mu_{\infty} t}$, (see [DK]) and hence $E(t) \leq c e^{-2 \mu_{\infty} t}$ by (4.7). Finally, using (4.8), we obtain

$$
\left|F^{A}\right|_{\tilde{g}} \leq c e^{-\mu_{\infty} t} \text { on }\left[0, \log \left(2 r_{0} / \lambda\right)\right] \times \mathbb{S}^{3} .
$$

Donaldson observed that the eigenvalue $\mu\left(\hat{g}_{s t d}\right)$ is precisely 2. Now $\mu(h)$ varies continuously with the metric on the sphere (with respect to the $C^{0}$ topology), and all the $\hat{g}_{t}$ can be made arbitrarily close to the standard one by taking $r_{0}$ sufficiently small. Hence we can replace $\mu_{\infty}$ in (4.15) by $2-\delta$, for any small but positive $\delta$, yielding (3.2). But we have actually shown more:

Proposition 4.1. If $\mu_{\infty} \geq 2$, then (3.3) holds on $B_{2 r_{0}}(p)$.

Theorem 3.2 will then follow from the following proposition.

Proposition 4.2. If $(M, g)$ satisfies the $\bigwedge_{-}^{2}$ curvature condition and $r_{0}$ is sufficiently small, then $\mu_{\infty}=2$.

Our proof essentially consists of calculating a directional derivative of $\mu(h)$ at the standard metric. Changing our notation slightly from (4.5), we consider a family of metrics of the form

$$
\hat{g}_{t}=\hat{g}_{s t d}-t R_{i k j l}(p) u^{k} u^{l} d u^{i} \otimes d u^{j}+O\left(t^{3 / 2}\right), \quad 0 \leq t \leq 2 r_{0},
$$

and we henceforth let the $t$ in $*_{t}, D_{t}$, and $\mu_{t}$ refer to the $t$ in (4.16). It suffices to prove that $\mu_{t} \geq 2$ for any such metric. (This will prove equality in Proposition 4.2 since, referring back to (4.5) and letting $\lambda \rightarrow 0$, we see that $\mu_{\infty} \leq \mu\left(\hat{g}_{s t d}\right)$.)

First we note that each operator $D_{t}: \Omega^{1}\left(\mathbb{S}^{3}\right) \rightarrow \Omega^{1}\left(\mathbb{S}^{3}\right)$ is self-adjoint (with respect to $\hat{g}_{t}$ ). If $D_{t} \omega=\nu \omega$ and $\nu \neq 0$ then $\omega$ is automatically co-closed, and 
hence an eigenform of the $\hat{g}_{t}$-Laplacian with eigenvalue $\nu^{2}$. Furthermore it is not hard to check that the spectrum consists only of eigenvalues. Thus, while $D_{t}$ is not elliptic, its spectrum is still discrete except for a zero eigenvalue of infinite multiplicity. In addition the family $D_{t}$ has a strong derivative $\dot{D}=-\dot{*} d$ at $t=0$, and $\left\|\left(D_{0}-t \dot{D}\right) \omega\right\|_{2} \leq c t^{1 / 2}\left\|D_{0} \omega\right\|_{2}$ for all $\omega, t$. It follows (cf. [Ka, Ch. $8 \S 2]$ ) that the derivative $\dot{\mu}$ of $\mu_{t}$ exists at $t=0$, and moreover that we can find a differentiable 1-parameter family of eigenforms $\omega_{t}$, with initial derivative $\dot{\omega}$, satisfying

$$
\begin{aligned}
& D_{t} \omega_{t}=-*_{t} d \omega_{t}=\mu_{t} \omega_{t}, \\
&\left\langle\omega_{t}, \omega_{t}\right\rangle_{L^{2}\left(\mathbb{S}^{3} ; \hat{g}_{s t d}\right)}=1
\end{aligned}
$$

for all small $t$. Differentiating (4.17-4.18) at $t=0$ we find

$$
\begin{aligned}
D_{0} \dot{\omega}-\dot{*} d \omega_{0} & =\dot{\mu} \omega_{0}+\mu_{0} \dot{\omega}, \\
\left\langle\omega_{0}, \dot{\omega}\right\rangle & =0 .
\end{aligned}
$$

(In (4.20) and below, all $L^{2}$ inner products are taken with respect to $\hat{g}_{s t d}$.) Taking inner product with $\omega_{0}$ on both sides of (4.19), and using self-adjointness of $D_{0}$, we find

$$
\dot{\mu}=-\left\langle\omega_{0}, \dot{*} d \omega_{0}\right\rangle=-\int_{\mathbb{S}^{3}} \omega_{0} \wedge * \dot{*} d \omega_{0} .
$$

But we also have $\left(*_{t}\right)^{2} \equiv \mathrm{Id}$, so $* \dot{*} d \omega_{0}=\dot{*}\left(-* d \omega_{0}\right)=\dot{*} \mu_{0} \omega_{0}$. Hence

$$
\dot{\mu} / \mu_{0}=-\int_{\mathbb{S}^{3}} \omega_{0} \wedge \dot{*} \omega_{0} .
$$

To prove Proposition 4.2 it suffices to show that $\dot{\mu}$ is positive. Therefore we are reduced to showing that, under the $\Lambda_{-}^{2}$ curvature condition, $\int_{\mathbb{S}^{3}} \omega \wedge \dot{*} \omega<0$ for any nonzero $\omega$ in the $\mu_{0}$-eigenspace (=2-eigenspace) of $D_{0}$.

We now regard $\left\{y^{i}\right\},\left\{u^{i}\right\}$ simply as functions on $\mathbb{R}^{4}$, with its standard metric and orientation (which we take to be consistent with the orientation of $M$ ). Letting $\Omega, *$ refer to the standard metric on the sphere, we have $\Omega=(1 / 6) \epsilon_{i j k l} u^{i} d u^{j} \wedge d u^{k} \wedge d u^{l}$ and $* d u^{i}=-(1 / 2) \epsilon_{i j k l} u^{j} d u^{k} \wedge d u^{l}$. It then easy to check that the 2-eigenspace of $D_{0}=-* d$ is the three-dimensional space

$$
\left\{\omega=\alpha_{i j} u^{i} d u^{j} \mid \alpha \in \bigwedge_{-}^{2}\left(\mathbb{R}^{4}\right)\right\}
$$


We next compute $\dot{*}$. For this it is convenient to view $\hat{g}_{t}$ as the restriction from $\mathbb{R}^{4}$ to $\mathbb{S}^{3}$ of the metric $g_{t}=\left(g_{t}\right)_{i j} d y^{i} \otimes d y^{j}$, where

$$
\left(g_{t}\right)_{i j}(y)=\delta_{i j}-t R_{i k j l} y^{k} y^{l}+O\left(t^{3 / 2}\right)
$$

and $R_{i k j l}=R_{i k j l}(p)$. The unit normals to the sphere are the same for each metric in this family. Temporarily writing $*_{t}^{(3)}, *_{t}^{(4)}$ for the star operators on $\mathbb{R}^{4}, \mathbb{S}^{3}$ respectively, and $j$ for the inclusion $\mathbb{S}^{3} \hookrightarrow \mathbb{R}^{4}$, we have

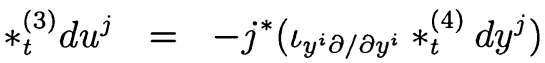

$$
\begin{aligned}
& =-j^{*}\left(g_{t}^{j k}\left|g_{t}\right|^{1 / 2} *_{0}^{(4)} d y^{k}\right) \\
& =j^{*}\left(g_{t}^{j k}\left|g_{t}\right|^{1 / 2}\right) *_{t}^{(3)} d u^{k} \text {. }
\end{aligned}
$$

Here $\left|g_{t}\right|$ stands for the determinant of the $4 \times 4$ matrix $\left(\left(g_{t}\right)_{i j}\right)$, and $\left(g_{t}^{i j}\right)$ for the inverse of this matrix. From (4.22) we have $\left|g_{t}\right|(u)=1-t R_{k l} u^{k} u^{l}+O\left(t^{3 / 2}\right)$ ( $R_{k l}$ being the components of the Ricci tensor) and $g_{t}^{i j}(u)=\delta_{i j}+t R_{i k j l} u^{k} u^{l}$. Thus, simply writing $*$ once again for $*_{0}^{(3)}$, we find

$$
\dot{*} d u^{j}=B_{j m k n} u^{m} u^{n} * d u^{k}
$$

where

$$
B_{j m k n}=R_{j m k n} * d u^{k}-\frac{1}{2} R_{m n} \delta_{j k}
$$

Now let $0 \neq \omega \in \Omega^{1}\left(\mathbb{S}^{3}\right)$ be as in (4.21). Since $\hat{g}_{s t d}\left(d u^{j}, d u^{q}\right)=\delta_{j q}-u^{j} u^{q}$ and $\alpha_{i j}$ is antisymmetric, we find

$$
\begin{aligned}
& \omega \wedge \dot{*} \omega=\alpha_{i j} \alpha_{k l} B_{l m q n} u^{i} u^{k} u^{m} u^{n} \hat{g}_{s t d}\left(d u^{j}, d u^{q}\right) \Omega \\
& \quad=\omega \wedge \dot{*} \omega=\alpha_{i j} \alpha_{k l} B_{l m j n} u^{i} u^{k} u^{m} u^{n} \Omega .
\end{aligned}
$$

We need one simple computational lemma.

\section{Lemma 4.3.}

$$
\int_{\mathbb{S}^{3}} u^{i} u^{k} u^{m} u^{n} \Omega=\frac{\pi^{2}}{12}\left(\delta_{i k} \delta_{m n}+\delta_{i m} \delta_{k n}+\delta_{i n} \delta_{k m}\right) .
$$

Proof. Since the integral over the sphere of any odd function of $u$ vanishes, by symmetry we are reduced to computing the integrals $I_{4,0}:=\int_{\mathbb{S}^{3}}\left(u^{1}\right)^{4}$ and $I_{2,2}:=\int_{\mathbb{S}^{3}}\left(u^{1}\right)^{2}\left(u^{2}\right)^{2}$. Let $V$ be the (standard) volume of $\mathbb{S}^{3}$; it is elementary that $V=2 \pi^{2}$. Using $\sum\left(u^{i}\right)^{2}=1$ and symmetry we have

$$
I_{4,0}=\int\left[\left(u^{1}\right)^{2}\left(1-\left(u^{2}\right)^{2}-\left(u^{3}\right)^{2}-\left(u^{4}\right)^{2}\right)\right]=V / 4-3 I_{2,2} \text {. }
$$


We then simply compute

$$
I_{4,0}=\int_{0}^{\pi} \cos ^{4} \theta \cdot 4 \pi \sin ^{2} \theta d \theta=\pi^{2} / 4 .
$$

Hence $I_{2,2}=\pi^{2} / 12$ and we are done.

Applying this to (4.23) we have

$$
\begin{aligned}
\frac{12}{\pi^{2}} \int_{\mathbb{S}^{3}} \omega \wedge \dot{*} \omega & =\left(\alpha_{i j} \alpha_{i k} B_{j m k m}+\alpha_{i j} \alpha_{k l}\left(B_{l i j k}+B_{l k j i}\right)\right) \\
& =\alpha_{i j} \alpha_{k l}\left(R_{l i j k}+R_{l k j i}\right)-\frac{1}{2} R \alpha_{i j} \alpha_{i j}
\end{aligned}
$$

where $R$ is the scalar curvature. Since $R_{l k j i}=R_{i j k l}$ and $\alpha_{k l} R_{l i j k}=\frac{1}{2} \alpha_{k l}\left(R_{l i j k}-\right.$ $\left.R_{k i j l}\right)=\frac{1}{2} \alpha_{k l}\left(-R_{i l j k}-R_{i k l j}\right)=\frac{1}{2} \alpha_{k l} R_{i j k l}$, we have $\alpha_{i j} \alpha_{k l}\left(R_{l i j k}+R_{l k j i}\right)=$ $\frac{3}{2} \alpha_{i j} \alpha_{k l} R_{i j k l}$. If we decompose the Riemann tensor into its Weyl, traceless Ricci, and scalar curvature pieces, then the traceless Ricci and the self-dual part of the Weyl curvature cannot contribute to this last expression, since $\alpha \in \Lambda_{-}^{2}\left(\mathbb{R}^{4}\right)$ and $\Lambda_{-}^{2}\left(\mathbb{R}^{4}\right) \otimes \Lambda_{-}^{2}\left(\mathbb{R}^{4}\right)$ does not contain the representations of $S O(4)$ corresponding to these parts of the curvature (cf. $\S 7$ ). Thus we can replace $R_{i j k l}$ in this expression by the sum of its anti-self-dual Weyl and scalar components, namely $W_{i j k l}^{-}+(R / 12)\left(\delta_{i k} \delta_{j l}-\delta_{i l} \delta_{j k}\right)$. The right-hand side of (4.24) therefore reduces to

$$
\frac{3}{2}\left(W_{i j k l}^{-} \alpha_{i j}-\frac{R}{6} \alpha_{k l}\right) \alpha_{k l}
$$

On the other hand, if we set $\tilde{\alpha}=(1 / 2) \alpha_{i j} d x^{i} \wedge d x^{j} \in \Lambda_{-}^{2} T_{p}^{*} M$ (where $\left\{x^{i}\right\}$ are our original normal coordinates based at $p$ ), then

$$
\begin{aligned}
g\left(\left(\frac{R}{6} I d-2 \mathcal{W}_{-}\right) \tilde{\alpha}, \tilde{\alpha}\right) & =\frac{1}{2}\left(\frac{R}{6} \alpha_{k l}-W_{i j k l}^{-} \alpha_{i j}\right) \alpha_{k l} \\
& =-\frac{4}{\pi^{2}} \int_{\mathbb{S}^{3}} \omega \wedge \dot{*} \omega
\end{aligned}
$$

by the calculation above. Under the $\bigwedge_{-}^{2}$ curvature condition, the left-hand side of (4.25) is positive. Thus $\int \omega \wedge \dot{*} \omega$ is negative, proving Proposition 4.2, and therefore Theorem 3.2 . 


\section{How CLOSE is $\tilde{H}_{A}$ TO $H_{A}$ ?}

With the estimates of $\S 3$ now justified we can begin to estimate the error introduced by localization, continuing where we left off at the end of $\S 2$. For this we need to quantify the how good an approximation of $H_{A}$ the space $\tilde{H}_{A}$ is; specifically, we need to bound $\left.\left(\mathrm{Id}-\pi_{A}\right)\right|_{\tilde{H}_{A}}$ somehow. It is clear that to estimate $\operatorname{num}(\pi \tilde{Z}, \pi \tilde{W} ; A)-\operatorname{num}(\tilde{Z}, \tilde{W} ; A)$ we need at least a bound on the norm of Id $-\pi_{A}$ as an operator from $L^{2}$ to $L^{4}$, but several more operator norms are relevant as well. The necessary estimates are summarized in Proposition 5.1 below. We remark that nothing in this section other than this proposition assumes the $\bigwedge_{-}^{2}$ curvature condition.

Notation. Given a vector field $Z$ and a connection $A \in \mathcal{M}_{\lambda_{0}}$, we will write

$$
\xi_{Z}^{A}=\pi_{A} \tilde{Z}^{A}-\tilde{Z}^{A} \quad \in \Omega^{1}(\operatorname{Ad} P) .
$$

When no confusion can arise, we will write $\xi_{Z}^{A}$ simply as $\xi_{Z}$.

Proposition 5.1. Assume that $(M, g)$ satisfies the $\Lambda_{-}^{2}$ curvature condition. Then there are constants $c$ such that for $A \in \mathcal{M}_{\lambda_{0}}$ and $\tilde{Z}=\tilde{Z}_{\mathbf{b}}+k \tilde{Z}^{\prime} \in \tilde{H}_{A}$,

(a)

$$
\begin{aligned}
\left\|\xi_{Z}^{A}\right\|_{2}+\left\|r \xi_{Z}^{A}\right\|_{4}+\left\|r \nabla^{A} \xi_{Z}^{A}\right\|_{2} & \leq c\left(|\mathbf{b}| \lambda^{2} \sqrt{|\log \lambda|}+|k| \lambda^{2}\right) \\
& \leq c\left(\lambda^{2} \sqrt{|\log \lambda|}\left\|\tilde{Z}_{\mathbf{b}}\right\|_{2}+\lambda\left\|k \tilde{Z}^{\prime}\right\|_{2}\right)
\end{aligned}
$$

(b)

$$
\begin{aligned}
\left\|\xi_{Z}^{A}\right\|_{4}+\left\|\nabla^{A} \xi_{Z}^{A}\right\|_{2} & \leq c(|\mathbf{b}| \lambda+|k| \lambda) \\
& \leq c\left(\lambda\left\|\tilde{Z}_{\mathbf{b}}\right\|_{2}+\left\|k \tilde{Z}^{\prime}\right\|_{2}\right)
\end{aligned}
$$

and (c)

$$
\left\|r \xi_{Z_{\mathrm{b}}}^{A}\right\|_{2}+\left\|r^{2} \nabla^{A} \xi_{Z_{\mathrm{b}}}^{A}\right\|_{2} \leq c|\mathbf{b}| \lambda^{2} \leq c \lambda^{2}\left\|\tilde{Z}_{\mathbf{b}}\right\|_{2}
$$

Remark 5.1. (1) As $\lambda \rightarrow 0,\left\|Z_{\mathbf{b}}\right\|_{2} \rightarrow 2 \pi|\mathrm{b}|$ and $\left\|\lambda^{-1} Z^{\prime}\right\|_{2} \rightarrow 2 \pi \sqrt{2}$ (see (2.1)), so we need prove only the first half of each inequality above.

(2) Part (a) improves the 'tangential' ( $\tilde{Z}_{\mathbf{b}}$ ) part of Proposition 3.7 of [GP2] by a full power of $\lambda$, and slightly improves the 'normal' $\left(\tilde{Z}^{\prime}\right)$ part. 
(3) For $\mathbb{S}^{4}$ the pointwise norm of $\left(\pi_{A}-\mathrm{Id}\right)\left(\tilde{Z}_{\mathbf{b}}+k Z^{\prime}\right)$ is bounded above and below by multiples of $\left(|\mathbf{b}| r^{2}+|k| r^{3}\right)|F|$; moreover Corollary 3.4 holds with the inequalities going both ways. This shows that the estimates in part (a) and (c) are sharp, as is the tangential part of the estimate in (b). But it also suggests that it may be possible to improve the coefficient of $|k|$ in (5.3) to $\lambda^{2} \sqrt{|\log \lambda|}$.

Our proof of Proposition 5.1 requires an observation from [GP2] concerning the approximate tangent space and the bilinear pairing $\downarrow: \Gamma\left(\operatorname{Sym}_{0}^{2}\left(T^{*} M\right)\right) \otimes$ $\Gamma\left(\bigwedge_{+}^{2}\left(T^{*} M\right)\right) \rightarrow \Gamma\left(\bigwedge_{-}^{2}\left(T^{*} M\right)\right)$ given by

$$
T \curvearrowleft \omega=T_{a b} \omega_{b c} \theta^{a} \wedge \theta^{c} .
$$

Here $\operatorname{Sym}_{0}^{2}\left(T^{*} M\right)$ is the bundle of traceless symmetric tensors; the above combination is always anti-self-dual whenever $\omega$ is self-dual. We use the same notation for the obvious extension of this pairing to $\omega \in \Lambda_{+}^{2}\left(T^{*} M\right) \otimes E$ for any auxiliary bundle $E$; the pairing then results in an anti-self-dual $E$-valued two-form.

Lemma 5.2 (Lemma 3.1 of [GP2]). Let $Z=\operatorname{grad} \phi$ be any gradient vector field and $A$ any self-dual connection. Then $d_{A}^{*} \tilde{Z}=0$ and $d_{A}^{-} \tilde{Z}=H^{0} \phi \natural F^{A}$, where $H^{0} \phi$ is the traceless part of the Hessian of $\phi$.

We apply this to $\tilde{Z} \in \tilde{H}_{A}$. Since $H_{A}=\operatorname{ker}\left(d_{A}^{*}\right) \cap \operatorname{ker}\left(d_{A}^{-}\right)$, and $d_{A}^{*} \tilde{Z}=0$, it follows that $\tilde{Z}-\pi_{A} \tilde{Z} \in\left(\operatorname{ker} d_{A}^{-}\right)^{\perp} \cap C^{\infty}=\operatorname{im}\left(d_{A}^{-}\right)^{*} \cap C^{\infty}$. Thus we can write

$$
\xi_{Z}^{A}=\tilde{Z}-\pi_{A} \tilde{Z}=\left(d_{A}^{-}\right)^{*} \eta
$$

for some $\eta \in \Omega_{-}^{2}(\operatorname{Ad} P)$. But if $Z=\operatorname{grad} \phi$, we also have $d_{A}^{-}\left(\tilde{Z}-\pi_{A} \tilde{Z}\right)=$ $d_{A}^{-} \tilde{Z}=H^{0} \phi \curvearrowleft F^{A}$. Putting this together with (5.6), we conclude that

$$
\Delta_{-}^{A} \eta=H^{0} \phi \natural F^{A} .
$$

This elliptic equation is the source of the bounds in Proposition 5.1. We begin with the following lemma.

Lemma 5.3. There are constants $c$ such that if $A \in \mathcal{M}_{\lambda_{0}}, \eta, \omega \in \Omega_{-}^{2}(\operatorname{Ad} P)$, and

$$
\Delta_{-}^{A} \eta=\omega
$$


then (a)

$$
\left\|\left(d_{A}^{-}\right)^{*} \eta\right\|_{2} \leq c\|r \omega\|_{2}
$$

(b)

$$
\left\|\left(d_{-}^{A}\right)^{*} \eta\right\|_{4}+\left\|\nabla^{A}\left(d_{-}^{A}\right)^{*} \eta\right\|_{2} \leq c\left(\|\omega\|_{2}+\lambda^{-1}\|r \omega\|_{2}\right),
$$

and $(\mathrm{c})$

$$
\left\|r\left(d_{-}^{A}\right)^{*} \eta\right\|_{4}+\left\|r \nabla^{A}\left(d_{-}^{A}\right)^{*} \eta\right\|_{2} \leq c\|r \omega\|_{2} .
$$

Proof. (a) Using (3.18)

$$
\left\|\left(d_{A}^{-}\right)^{*} \eta\right\|_{2}^{2}=\left\langle\eta, \Delta_{-}^{A} \eta\right\rangle=\left\langle r^{-1} \eta, r \omega\right\rangle \leq c\left\|\left(d_{A}^{-}\right)^{*} \eta\right\|_{2}\|r \omega\|_{2},
$$

and (5.8) follows.

(b) From the Sobolev embedding $L_{1}^{2} \hookrightarrow L^{4}$ we have

$$
\left\|\left(d_{-}^{A}\right)^{*} \eta\right\|_{4}^{2} \leq c\left(\left\|\left(d_{-}^{A}\right)^{*} \eta\right\|_{2}^{2}+\left\|\nabla^{A}\left(d_{-}^{A}\right)^{*} \eta\right\|_{2}^{2}\right) .
$$

Using the Weitzenböck identity (2.12) and the fact that $d^{A^{*}} \circ d_{-}^{A^{*}}=0$ for self-dual connections, we have

$$
\begin{aligned}
\left\|\nabla^{A}\left(d_{-}^{A}\right)^{*} \eta\right\|_{2}^{2} & =\left\langle\left(d_{-}^{A}\right)^{*} \eta, \Delta^{A}\left(d_{-}^{A}\right)^{*} \eta\right\rangle \\
& =\left\langle\left(d_{-}^{A}\right)^{*} \eta, 2\left(d_{-}^{A}\right)^{*} \Delta_{-}^{A} \eta+\mathcal{R}\left(\left(d_{-}^{A}\right)^{*} \eta\right)+2\left[F,\left(d_{-}^{A}\right)^{*} \eta\right]\right\rangle \\
& \leq c\left(\left\langle\Delta_{-}^{A} \eta, \Delta_{-}^{A} \eta\right\rangle+\left\|\left(d_{-}^{A}\right)^{*} \eta\right\|_{2}^{2}+\|F\|_{4}\left\|\left(d_{-}^{A}\right)^{*} \eta\right\|_{2}\left\|\left(d_{-}^{A}\right)^{*} \eta\right\|_{4}\right) .
\end{aligned}
$$

Thus

$\left\|\left(d_{-}^{A}\right)^{*} \eta\right\|_{4}^{2}+\left\|\nabla^{A}\left(d_{-}^{A}\right)^{*} \eta\right\|_{2}^{2} \leq c\left(\langle\omega, \omega\rangle+\left\|\left(d_{-}^{A}\right)^{*} \eta\right\|_{2}^{2}+\|F\|_{4}^{2}\left\|\left(d_{-}^{A}\right)^{*} \eta\right\|_{2}^{2}\right)+\frac{1}{2}\left\|\left(d_{-}^{A}\right)^{*} \eta\right\|_{4}^{2}$, implying

$$
\left\|\left(d_{-}^{A}\right)^{*} \eta\right\|_{4} \leq c\left(\|\omega\|_{2}+\left\|\left(d_{-}^{A}\right)^{*} \eta\right\|_{2}\left(1+\|F\|_{4}\right)\right) .
$$

By part (a), we can replace $\left\|\left(d_{-}^{A}\right)^{*} \eta\right\|_{2}$ in this inequality by $\|r \omega\|_{2}$. From Corollary 3.4, the $L^{4}$ norm of $F$ over $B_{2 r_{0}}(p)$ is $\leq c \lambda^{-1}$. (This does not require the $\Lambda_{-}^{2}$ curvature condition; see the remark after Corollary 3.4.) But as $\lambda \rightarrow 0$, the sup-norm of $F$ outside $B_{r_{0}}(p)$ goes to 0 uniformly in $\lambda$ (cf. [D1, Theorem 13.(ii)]), so $\|F\|_{4} \leq c \lambda^{-1}$, and using (5.8) we obtain (5.9). 
For (c), we proceed as in (b), first obtaining

$$
\begin{aligned}
\left\|r\left(d_{-}^{A}\right)^{*} \eta\right\|_{4}^{2} & \leq c\left(\left\|r\left(d_{-}^{A}\right)^{*} \eta\right\|_{2}^{2}+\left\|r\left(d_{-}^{A}\right)^{*} \eta\right\|_{4}+\left\|\nabla^{A} r\left(d_{-}^{A}\right)^{*} \eta\right\|_{4}^{2}\right) \\
& \leq c\left(\left\|\left(d_{-}^{A}\right)^{*} \eta\right\|_{2}^{2}+\left\|r \nabla^{A}\left(d_{-}^{A}\right)^{*} \eta\right\|_{2}^{2}\right) .
\end{aligned}
$$

Integrating by parts and using the Weitzenböck formula again, we have

$$
\begin{aligned}
\left\|r \nabla^{A}\left(d_{-}^{A}\right)^{*} \eta\right\|_{2}^{2}= & \left\langle d_{-}^{A}\left(r^{2} d_{-}^{A^{*}} \eta\right), d_{-}^{A^{*}} \eta\right\rangle \\
\leq & c\left(\left\|r \nabla^{A}\left(d_{-}^{A}\right)^{*} \eta\right\|_{2}\left\|_{2}\right\|\left(d_{-}^{A}\right)^{*} \eta \|_{2}+\left|\left\langle\Delta_{-}^{A} \eta, d_{-}^{A}\left(r^{2} d_{-}^{A^{*}} \eta\right)\right\rangle\right|\right. \\
& \left.\left.+\| d_{-}^{A^{*}} \eta\right)\left\|_{2}^{2}+\right\| r F\left\|_{4}\right\| r d_{-}^{A^{*}} \eta\left\|_{4}\right\| d_{-}^{A^{*}} \eta \|_{2}\right) .
\end{aligned}
$$

Combining this with the previous inequality and using part (a), we find

$$
\left\|r\left(d_{-}^{A}\right)^{*} \eta\right\|_{4}^{2}+\left\|r \nabla^{A}\left(d_{-}^{A}\right)^{*} \eta\right\|_{2}^{2} \leq c\|r \omega\|_{2}\left(1+\|r F\|_{4}\right) .
$$

Using Corollary 3.4 and the fact that $F$ is uniformly small for $r>r_{0}$, we find $\|r F\|_{4} \leq c$, which completes the proof.

To apply this to Proposition 5.1, we need one more lemma. We remark that while this lemma is valid for all $r$, only the behavior as $r \rightarrow 0$ will concern us later.

Lemma 5.4. There are constants $c$ for which the vector fields $Z_{\mathbf{a}}, Z^{\prime}$ satisfy

$$
\begin{aligned}
\left|Z_{\mathbf{a}}\right|+\left|\Delta Z_{\mathbf{a}}\right| & \leq c|\mathbf{a}|, \\
\left|\nabla Z_{\mathbf{a}}\right| & \leq c|\mathbf{a}| r, \\
\left|Z^{\prime}\right|+\left|\Delta Z^{\prime}\right| & \leq c r, \quad \text { and } \\
\left|\nabla Z^{\prime}\right| & \leq c .
\end{aligned}
$$

Proof. Let $\left\{x^{i}\right\}$ be normal coordinates centered at $p$ and let $R_{i j k l}$ be the components of the Riemann tensor at $p$. From Definition 2.1, in these coordinates we have $Z_{\mathbf{a}}=a_{i} \operatorname{grad}\left(\beta^{3} x^{i}\right)$ and $Z^{\prime}=\frac{1}{2} \operatorname{grad}\left(\beta^{3} r^{2}\right)$.From the Taylor expansion of the metric and Christoffel symbols in normal coordinates,

$$
\begin{gathered}
g_{i j}(x)=\delta_{i j}-\frac{1}{3} R_{\text {minj }} x^{m} x^{n}+\cdots, \\
\Gamma_{j k}^{i}(x)=-\frac{1}{3}\left(R_{i j k l}+R_{i k j l}\right) x^{l}+\cdots,
\end{gathered}
$$


we have

$$
\begin{aligned}
\operatorname{grad} x^{i} & =\frac{\partial}{\partial x^{i}}+O\left(r^{2}\right) \\
\nabla \operatorname{grad} x^{i} & =\frac{1}{3}\left(R_{i j k l}+R_{i k j l}\right) x^{l} \frac{\partial}{\partial x^{j}} \otimes d x^{k}+O\left(r^{2}\right), \\
\nabla \nabla \operatorname{grad} x^{i} & =\frac{1}{3}\left(R_{i j k l}+R_{i k j l}\right) \frac{\partial}{\partial x^{j}} \otimes d x^{k} \otimes d x^{l}+O(r), \\
\operatorname{grad} \frac{1}{2} r^{2} & =x^{i} \frac{\partial}{\partial x^{i}}, \\
\nabla \operatorname{grad} \frac{1}{2} r^{2} & =\frac{\partial}{\partial x^{i}} \otimes d x^{i}+O\left(r^{2}\right), \quad \text { and } \\
\nabla \nabla \operatorname{grad} \frac{1}{2} r^{2} & =O(r) .
\end{aligned}
$$

Since $\beta \equiv 1$ in a ball of fixed size near $p$ its presence in $Z_{\mathbf{a}}$ and $Z^{\prime}$ does not affect the order of growth. Since $\Delta Z=-\operatorname{tr}_{g}(\nabla \nabla Z)$, the results follow.

Proof of Proposition 5.1, part (a). Let $\tilde{Z}=\tilde{Z}_{\mathbf{b}}+k \tilde{Z}^{\prime} \in \tilde{H}_{A}$, and let $\eta$ be as in (5.6). Then (5.7) holds with $\phi=\phi_{\mathrm{b}}+k \phi^{\prime}$, and equations (5.13) and (5.15) imply $\left|H^{0} \phi\right| \leq c\left(|\mathbf{b}| r+|k| r^{2}\right)$. Let $\omega=H^{0} \phi \curvearrowleft F$; from Corollary 3.4 we then have $\|r \omega\|_{2} \leq c\left(|\mathbf{b}| \lambda^{2} \sqrt{|\log \lambda|}+|k| \lambda^{2}\right)$. Using Lemma 5.3 we obtain (5.2).

From Lemmas 5.3 and 5.4 one can quickly obtain a weaker version of (5.3), namely

$$
\left\|\xi_{Z}^{A}\right\|_{4}+\left\|\nabla^{A} \xi_{Z}^{A}\right\|_{2} \leq c(|\mathbf{b}| \lambda \sqrt{|\log \lambda|}+|k| \lambda)
$$

as well as a similar weaker verion of (5.4). However, these inequalities are not sharp. This can be seen by examining what happens on $\mathbb{S}^{4}$, using the pointwise bound mentioned in Remark 5.1 (3). The approach we will take to prove parts (b) and (c) of Proposition 5.1 grew out of an attempt to generalize this pointwise estimate and thereby obtain sharp inequalities.

If we take $Z=Z_{\mathrm{b}}, \phi=\phi_{\mathrm{b}}$ in (5.6-5.7), the right-hand side of (5.7) is $O(|\mathrm{~b}| r|F|)$ and is concentrated near $r=0$, so one might optimistically hope that $\eta$ is $O\left(|\mathbf{b}| r^{3}|F|\right)$ and that $\left(d_{A}^{-}\right)^{*} \eta$ is $O\left(|\mathbf{b}| r^{2}|F|\right)$. Were this true, Proposition 5.1 would follow from Corollary 3.4, but such a simple pointwise bound is wishful thinking. However, Corollary 3.4 shows that, as far as $L^{4}$ estimates involving $F$ are concerned, a power of $r$ effectively cancels a derivative; thus 
any pointwise estimate of the form

$$
\left|\left(d_{-}^{A}\right)^{*} \eta\right| \leq c|\mathbf{b}| \sum_{j=0}^{N} r^{j+2}\left|\left(\nabla^{A}\right)^{j} F^{A}\right|
$$

would do just as well. This is still too much to ask, but it turns out that with $N=3$ we can correct the right-hand side by adding a non-local term which still has sufficiently small norms. This assertion is far from obvious, and we devote the next two sections to proving it and examining some consequences.

\section{The EQUATION $\Delta_{-}^{A} \eta=H^{0} \phi_{b} \natural F^{A}$}

For notational simplicity, throughout this section we fix the center point $p$ of $A$ and fix a positively oriented normal coordinate system $\left\{x^{i}\right\}$ based at $p$; as usual, our estimates remain independent of these choices. Any components $R_{i j k l}$ of the Riemann tensor we write down are assumed to be evaluated at $p$ in normal coordinates. Except where noted, we do not assume the $\bigwedge_{-}^{2}$ curvature condition.

Our approach to (approximately) solving

$$
\Delta_{-}^{A} \eta=H^{0} \phi_{\mathrm{b}} \natural F^{A}
$$

involves a combination of analysis and representation theory. To motivate the approach, let us ignore for a moment the presence of the cutoff function $\beta$; essentially we are then working on $\mathbb{R}^{4}$, but with a metric pulled back from $M$ by the coordinates $\left\{x^{i}\right\}$. The components of all tensors we write henceforth will be written relative to the local basis $\left\{d x^{i}\right\}$ of $T^{*} M$. For now we will take the linear function $f_{\mathrm{b}}$ to be some particular $x^{\mu}$. The Hessian $H x^{\mu}$ is $\nabla d x^{\mu}=-\Gamma_{j k}^{\mu} d x^{j} \otimes d x^{k}$, so, using (5.13), we have

$$
H^{0} x^{\mu}=\frac{1}{6}\left[2\left(R_{\mu j k i}+R_{\mu k j i}\right)+R_{\mu i} \delta_{j k}\right] x^{i} d x^{j} \otimes d x^{k}+O\left(r^{2}\right),
$$

where $R_{i j}=R^{\nu}{ }_{i \nu j}$ are the components of the Ricci tensor. Thus $H^{0} x^{\mu}$ is a traceless symmetric tensor whose components, to leading order, are linear polynomials in normal coordinates. It is reasonable to guess that the solution of (6.1) will be of the form $T \natural F^{A}+$ 'higher order terms', where $T$ is a traceless 
symmetric tensor whose components, in normal coordinates, are homogeneous polynomials of degree three. Using the Weitzenböck formula (2.13), we have

$$
2 \Delta_{-}^{A}\left(T \natural F^{A}\right)=\mathcal{R}\left(T, F^{A}\right)+\Delta T \natural F^{A}+T \natural \Delta^{A} F^{A}-2 \nabla T \natural \nabla^{A} F^{A},
$$

where $\nabla T \curvearrowleft F^{A}=g^{i j} \nabla_{i} T \natural \nabla^{A}{ }_{j} F^{A}$. Assuming the homogeneity above, $\Delta T$ is a traceless symmetric tensor whose components are linear terms plus $O\left(r^{2}\right)$; let us suppose for now that we can choose $T$ so that the linear terms agree with those of $H^{0} x^{\mu}$. We will see later that every term on the right-hand side of (6.3) except the last is already sufficiently small in all the norms that we need. Unfortunately this one bad term remains. We can try to cancel it by adding to our approximate solution a term of the form $S^{i} \emptyset \nabla^{A}{ }_{i} F^{A}$, or, equivalently, $S \curvearrowleft \nabla^{A} F^{A}$, where the components of $S \in \Gamma\left(\operatorname{Sym}_{0}^{2}\left(T^{*} M\right) \otimes\right.$ $T^{*} M$ ) are homogeneous polynomials of degree 4. It turns out that $S$ can be chosen to cancel the previous bad term, but a new bad term arises of the form $\nabla S \curvearrowleft \nabla^{A} \nabla^{A} F^{A}$. We can continue to play this game, hoping that it eventually terminates. Indeed it does so after one more term is added, essentially for representation-theoretic reasons.

To explain this, we first extend the definition of $\downarrow$. Writing

$$
\left(\nabla^{A}\right)^{k} F^{A}=F_{a b ; i_{1} \ldots i_{k}}\left(d x^{a} \wedge d x^{b}\right) \otimes d x^{i_{1}} \otimes \ldots \otimes d x^{i_{k}}
$$

given a tensor $V \in \operatorname{Sym}_{0}^{2}\left(T^{*} M\right) \otimes\left(T^{*} M\right)^{k}$ (the last factor meaning the $k$-fold tensor product of $T^{*} M$ with itself), we set

$$
V \curvearrowleft\left(\nabla^{A}\right)^{k} F^{A}=V_{a}^{b i_{1} \ldots i_{k}} F_{b c ; i_{1} \ldots i_{k}} \theta^{a} \wedge \theta^{c} \in \Gamma\left(\bigwedge_{-}^{2}\left(T^{*} M\right) \otimes \operatorname{Ad} P\right)
$$

(Indices are raised using $g^{i j}$ here.) Next we note that a normal coordinate system determines an isomorphism

$$
\iota_{k, m}: \operatorname{Sym}_{0}^{2}\left(\mathbb{R}^{4}\right) \otimes\left(\mathbb{R}^{4}\right)^{k} \otimes \operatorname{Sym}^{m}\left(\mathbb{R}^{4}\right) \rightarrow \Gamma\left(\operatorname{Sym}_{0}^{2}\left(T^{*} M\right) \otimes\left(T^{*} M\right)^{k}\right) \otimes P_{m}
$$

where $P_{m} \subset C^{\infty}\left(B_{r_{0}}(p)\right)$ is the space of polynomials homogeneous of degree $m$ in normal coordinates based at $p$. This map is defined by sending the standard basis of $\mathbb{R}^{4}$ to the local basis of sections $\left\{d x^{i}\right\}$ of $\Gamma\left(T^{*} M\right)$ in the first two factors in the image and to the coordinates $\left\{x^{i}\right\}$ themselves in the 
third factor. Given an $S O(4)$-equivariant linear map $L: \operatorname{Sym}_{0}^{2}\left(\mathbb{R}^{4}\right) \otimes \mathbb{R}^{4} \rightarrow$ $\operatorname{Sym}_{0}^{2}\left(\mathbb{R}^{4}\right) \otimes\left(\mathbb{R}^{4}\right)^{k} \otimes \operatorname{Sym}^{m}\left(\mathbb{R}^{4}\right)$ (where $S O(4)$ acts in the way induced from the standard representation on $\left.\mathbb{R}^{4}\right)$, we then set

$$
\hat{L}=\iota_{k, m} \circ L \circ \iota_{1,0}^{-1}: \operatorname{Sym}_{0}^{2}\left(T^{*} M\right) \otimes P_{1} \rightarrow \operatorname{Sym}_{0}^{2}\left(T^{*} M\right) \otimes\left(T^{*} M\right)^{k} \otimes P_{m} ;
$$

by equivariance, $\hat{L}$ is independent of the choice of normal coordinates.

Theorem 6.1. For $k \in\{0,1,2\}$ there exist $S O(4)$-equivariant linear maps $L^{(k)}: \operatorname{Sym}_{0}^{2}\left(\mathbb{R}^{4}\right) \otimes \mathbb{R}^{4} \rightarrow \operatorname{Sym}_{0}^{2}\left(\mathbb{R}^{4}\right) \otimes\left(\mathbb{R}^{4}\right)^{k} \otimes \operatorname{Sym}^{k+3}\left(\mathbb{R}^{4}\right)$ with the following property. Let $T=\hat{L}^{(0)}\left(\mathcal{H}_{\mathbf{b}}\right), S=\hat{L}^{(1)}\left(\mathcal{H}_{\mathbf{b}}\right), U=\hat{L}^{(2)}\left(\mathcal{H}_{\mathbf{b}}\right)$, where $\mathcal{H}_{\mathbf{b}}$ is the 1-jet of $H^{0} f_{\mathrm{b}}$ at $p$, identified in the obvious way as an element of $\operatorname{Sym}_{0}^{2}\left(T^{*} M\right) \otimes P_{1}$. Let $E$ be a vector bundle over $M$ with self-dual connection $A$. Then

$$
\Delta_{-}^{A}\left\{\beta^{3}\left(T \curvearrowleft F^{A}+S \natural \nabla^{A} F^{A}+U \curvearrowleft \nabla^{A} \nabla^{A} F^{A}\right)\right\}=H^{0} \phi_{\mathbf{b}} \natural F^{A}-R^{(\mathbf{b})},
$$

where the remainder $R^{(\mathbf{b})}$ satisfies

$$
\begin{aligned}
\left|R^{(\mathbf{b})}\right| \leq c \beta|\mathbf{b}|\left\{r^{2}\left|F^{A}\right|+\sum_{j=0}^{2}(\right. & r^{j+4}\left|\left(\nabla^{A}\right)^{j+1} F^{A}\right| \\
& \left.\left.+r^{j+3}\left|F^{A}\right|\left|\left(\nabla^{A}\right)^{j} F^{A}\right|\right)+r^{5}\left|\nabla^{A} F^{A}\right|^{2}\right\}
\end{aligned}
$$

pointwise. If $(M, g)$ is Einstein and its Weyl curvature tensor is anti-self-dual, this can be simplified; specifically, we can take $U=0$, omit $j=2$ from the sum in (6.7), and omit the last term in (6.7). On any ball of radius less than the injectivity radius of $M,(6.6)-(6.7)$ hold with the $\beta$ 's absent. The constant $c$ in the remainder bound depends only on the geometry of the compact manifold $M$; it is independent of the center point $p$, the connection $A$, and the bundle E.

Note the universality asserted in the above theorem. No particular Lie group is assumed to underlie the bundle $E$, and the connection $A$ is not assumed to be centered at $p$.

Before proving this theorem we see what it buys us. 
Proof of Proposition 5.1 completed, assuming Theorem 6.1. Because of (5.16) it suffices to take $k=0$ in (5.3). For $A \in \mathcal{M}_{\lambda_{0}}$, Theorem 6.1 implies that if $\eta$ solves (6.1), then

$$
\eta=\eta_{1}+G_{-}\left(R^{(\mathbf{b})}\right)
$$

where

$$
\eta_{1}=\beta^{3}(T \curvearrowleft F+S \natural \nabla F+U \natural \nabla \nabla F) .
$$

From the homogeneity of $T, S$, and $U$, we then have

$$
\left\|\left(d_{A}^{-}\right)^{*} \eta_{1}\right\|_{4} \leq c|\mathbf{b}| \sum_{j=0}^{3}\left\|\beta r^{j+2}\left(\nabla^{A}\right)^{j} F\right\|_{4} \leq c|\mathbf{b}| \lambda
$$

by Corollary 3.4 (it is here we assume the $\bigwedge_{-}^{2}$ curvature condition). Furthermore, using (5.9) we have

$$
\left\|\left(d_{A}^{-}\right)^{*} G_{-}^{A}\left(R^{(\mathbf{b})}\right)\right\|_{4} \leq c\left(\left\|R^{(\mathbf{b})}\right\|_{2}+\lambda^{-1}\left\|r R^{(\mathbf{b})}\right\|_{2}\right) .
$$

But

$$
\begin{aligned}
\left\|R^{(\mathbf{b})}\right\|_{2} \leq & c|\mathbf{b}|\left(\left\|r^{2} F\right\|_{2}+\sum_{j=0}^{2}\left(\left\|r^{j+4} \nabla^{j+1} F\right\|_{2}\right.\right. \\
& \left.\left.+\left\|r^{3 / 2} F\right\|_{4}\left\|r^{j+3 / 2} \nabla^{j} F\right\|_{4}\right)+\left\|r^{5 / 2} \nabla F\right\|_{4}^{2}\right) \\
\leq & c|\mathbf{b}| \lambda,
\end{aligned}
$$

and similarly

$$
\left\|r R^{(\mathbf{b})}\right\|_{2} \leq c|\mathbf{b}| \lambda^{2}
$$

so $\left\|\left(d_{-}^{A}\right)^{*} \eta\right\|_{4} \leq c|\mathbf{b}| \lambda$. The same approach works for $\left\|\nabla^{A}\left(d_{-}^{A}\right)^{*} \eta\right\|_{2}$, and we obtain (5.3). For (5.4) we have

$$
\begin{aligned}
\left\|r d_{-}^{A^{*}} \eta\right\|_{2} & \leq\left\|r d_{-}^{A^{*}} \eta_{1}\right\|_{2}+\left\|d_{-}^{A^{*}} G_{-}^{A}\left(R^{(\mathbf{b})}\right)\right\|_{2} \\
& \leq c\left(|\mathbf{b}| \lambda^{2}+\left\|r R^{(\mathbf{b})}\right\|_{2}\right) \\
& \leq c|\mathbf{b}| \lambda^{2}
\end{aligned}
$$

and the argument bounding $\left\|r^{2} \nabla^{A} d_{-}^{A^{*}} \eta\right\|_{2}$ is similar.

We remark that an analog of Theorem 6.1 for $\phi^{\prime}$ instead of $\phi_{\mathbf{b}}$, with one higher power of $r$, would suffice to improve the coefficient of $|k|$ in (5.3) to $\lambda^{2}|\log \lambda|^{1 / 2}$ as discussed earlier. But, to the author's surprise, so far the method used below to prove Theorem 6.1 has failed to prove this analog. 
We begin the proof of Theorem 6.1 by noting (as in the proof of Lemma 5.4 ), that since $\beta \equiv 1$ near $p$, it does not affect any order of growth. Hence it suffices to prove (6.6-6.7) with the $\beta$ 's replaced by 1 , a simplification we make henceforth.

We will compare the left-hand side of (6.6) to what we would get if the Levi-Civita connection were replaced in certain terms by the flat connection $\partial$ determined by the $\left\{x^{i}\right\}$. We define $|T|=\max _{I, i, j}\left\{\left|c_{I i j}\right|\right\}$, where the coefficients $c_{I i j}$ are given by $T=c_{I i j} x^{I} d x^{i} \otimes d x^{j}$; here $I$ denotes a triple-index and $x^{I}$ denotes the corresponding cubic monomial. We define $|S|$ and $|U|$ similarly; $|F|,|\nabla F|, \ldots$, denote the pointwise norms of $F$ and its covariant derivatives. Examining the terms in (6.3), and noting that as $r \rightarrow 0, T=O\left(r^{3}|T|\right)$ (with corresponding statements about derivatives), we have

$$
\begin{aligned}
\Delta T & =-\partial_{i} \partial_{i} T+O\left(r^{3}|T|\right), \\
\nabla^{*} \nabla F & =O\left(|F|^{2}+|F|\right), \\
\nabla T & =\partial T+O\left(r^{4}|T|\right) .
\end{aligned}
$$

(We have used (3.6) in the middle equation.) Hence

$$
\begin{aligned}
& \mathcal{R}(T, F)=O\left(r^{3}|T||F|\right) \\
& \Delta T \natural F=-\partial_{i} \partial_{i} T \text { দ } F+O\left(r^{3}|T||F|\right) \\
& T \text { দ } \nabla^{*} \nabla F=O\left(r^{3}|T|\left(|F|+|F|^{2}\right)\right) \\
& \nabla T \curvearrowleft \nabla F=\partial T \text { Ł } \nabla F+O\left(r^{4}|T||\nabla F|\right) \text {. }
\end{aligned}
$$

In these equations, we are still using the definition (6.4), which involves the metric $g$. Let bh denote the corresponding pairing defined using the flat metric in normal coordinates. Putting all of the above together, we then find $2 \Delta_{-}^{A}(T$ Ł $F)=-\partial_{i} \partial_{i} T$ 蚔 $F-2 \partial T$ 如 $\nabla^{A} F+O\left(|T|\left(r^{3}|F|+r^{4}|\nabla F|+r^{3}|F|^{2}\right)\right)$.

The Laplacian of $S \curvearrowleft \nabla F$ is handled in a similar fashion, with a little more work required to deal with $S \sharp \nabla^{*} \nabla(\nabla F)$. To estimate this term we use Lemma 3.1, with $E=\operatorname{Ad} P \otimes \Lambda^{2} T^{*} M$; the curvature $K$ is then of the form $F^{A} \otimes 1+1 \otimes \mathcal{R}$. In our case, the derivatives of $F$ involved in $\nabla^{*} K$ can be ignored, since $A$ is Yang-Mills (though these terms would in any case be 
comparable to other terms that do not vanish). Applying the lemma, and again using the Weitzenböck formula (3.6), we find

$$
\begin{aligned}
S \natural \nabla^{*} \nabla(\nabla F) & =S \text { দR }(F)+\mathcal{R}(\nabla F)-4[F, \nabla F]\rangle \\
& =O\left(|S| r^{4}(|F|+|\nabla F|+|F||\nabla F|)\right) .
\end{aligned}
$$

Putting all of the terms involving $S$ together, we obtain

$$
\begin{aligned}
2 \Delta_{-}^{A}(S \text { Ł } \nabla F)=-\partial_{i} \partial_{i} S & \text { 蚔 } \nabla F-2 \partial S \text { 如 } \nabla \nabla F \\
& +O\left(|S|\left(r^{4}|F|+r^{4}|\nabla F|+r^{5}|\nabla \nabla F|+r^{4}|F||\nabla F|\right)\right) .
\end{aligned}
$$

The Laplacian of $U \curvearrowleft \nabla \nabla F$ is treated identically, except that Lemma 3.1 is used twice to commute a Laplacian through two derivatives. Putting everything together, we find

$$
\begin{aligned}
& 2 \Delta_{-}^{A}(T \curvearrowleft F+S \natural \nabla F+U \text { দ } \nabla \nabla F) \\
& =\left\{-\partial_{i} \partial_{i} T \text { 虬 } F-\partial_{i} \partial_{i} S-2 \partial T \text { 虬 } \nabla F+-\partial_{i} \partial_{i} U\right. \\
& \text { (6.8) } \quad-2 \partial S \text { 蛞 } \nabla \nabla F-2 \partial U \text { 蛞 } \nabla \nabla \nabla F\} \\
& +O\left(( | T | + | S | + | U | ) \left(r^{3}|F|+r^{4}|\nabla F|+r^{5}|\nabla \nabla F|+r^{6}|\nabla \nabla \nabla F|\right.\right. \\
& \left.\left.+r^{3}|F|^{2}+r^{4}|F||\nabla F|+r^{5}|F||\nabla \nabla F|+r^{5}|\nabla F|^{2}\right)\right) \text {. }
\end{aligned}
$$

To establish (6.6), we must show that the terms in curly brackets on the right-hand side of (6.8) can be chosen to give $2 H^{0} f_{\mathrm{b}}$ h $F$, modulo the remainder (6.7), and that $|T|+|S|+|U| \leq c|\mathbf{b}|$. (The second-order terms in $H^{0} f_{\mathbf{b}}$ are the reason the first term in (6.7) is $r^{2} F$ rather than the $r^{3} F$ in (6.8).) This rather long representation-theoretic argument is given in the next section. We will also see there why $U$ can be taken to be zero if $(M, g)$ is Einstein and anti-self-dual; the simplified remainder bound then follows by looking at the above argument and keeping track of the terms that arise only from $T$ and $S$.

\section{REPRESENTATION THEORY AND THE PARAMETRIX}

In this section we complete the proof of Theorem 6.1. In view of (6.8) it now suffices to show that there exist maps $L^{(k)}$ as in the statement of the theorem 
such that we can solve the following system of equations:

$$
\begin{aligned}
-\frac{1}{2} \Delta T & =\mathcal{H}_{\mathbf{b}} ; \\
\frac{1}{2} \Delta S-\partial T \natural \nabla F & =0 ; \\
\frac{1}{2} \Delta U-\partial S \curvearrowleft \nabla \nabla F & =\text { harmless terms; } \\
\partial U \curvearrowleft \nabla \nabla \nabla F & =\text { harmless terms. }
\end{aligned}
$$

Here "harmless terms" mean terms of the form in (6.7). For simplicity, in the above formula we have renamed the operation 妕 of (6.8) by $\curvearrowleft$-in this section there are no longer two such operations to distinguish-and have written $-\Delta$ for $\partial_{i} \partial_{i}$. We will drop the subscript $A$ from $F$ and $\nabla$ throughout, and abbreviate spaces such as $\operatorname{Sym}^{k}\left(\mathbb{R}^{4}\right)$ simply as $\operatorname{Sym}^{k}$. The ball $B_{2 r_{0}}(p)$ will simply be called $B$. The methods in this section are almost entirely algebraic; the manifold $M$, the metric $g$, and the bundle $E$ are essentially irrelevant.

The outline of the argument is this. First we show that (7.1-7.4) can be reduced to an inhomogeneous system of linear equations whose variables are the components of $T, S$, and $U$. This system is too large to be analyzed by simple techniques; there are roughly 9,000 unknowns, and-if we replace the right-hand sides of (7.3-7.4) by zeroes-over 15,000 equations. It is difficult even to determine the rank of the system, let alone whether it admits a solution. Our strategy is to analyze this system in terms of the representations of $S O(4)$, using Schur's lemma to reduce the number of equations as well as the number of variables drastically. Using this approach, we find that if we set $S$ and $U$ equal to zero, the system is overdetermined and the inhomogeneous system admits no solution. If we allow $S$ to be nonzero but not $U$, both the number of variables and the number of equations increases; what is not obvious is that because we are interested in the right-hand sides of (7.3-7.4) only modulo 'harmless' terms, the former number grows faster than the latter, and in fact we end up with more variables than equations. Unfortunately, the inhomogeneous system remains overdetermined and still does not admit a solution, save under the special curvature hypotheses mentioned in Theorem 6.1. Finally, by allowing $U$ to be nonzero we are able to obtain a solution in all cases. We end up having to show only that three matrices-a $2 \times 2$, a $3 \times 3$, 
and a $4 \times 4$-are nonsingular.

To present the argument in detail we first recall some elementary facts concerning the representations of $S O(4)$. The Lie algebra $\mathfrak{s o ( 4 )}$ splits as $\mathfrak{s o}(3) \oplus \mathfrak{s o}(3)$; we call $\mathfrak{s o}(3)_{ \pm}$the summand that acts trivially on $\Lambda_{\mp}^{2}$ under the action induced by the standard representation of $\mathfrak{s o}(4)$. The irreducible representations of the complexified Lie algebra $\mathfrak{s o}(4, \mathbb{C})=\mathfrak{s l}(2, \mathbb{C})_{+} \oplus \mathfrak{s l}(2, \mathbb{C})_{-}$ are of the form $V_{+} \otimes V_{-}$, where $V_{ \pm}$is an irreducible representation of the $\mathfrak{s l}(2, \mathbb{C})_{ \pm}$. Thus the irreducible representations of $\mathfrak{s o}(4, \mathbb{C})$ are classified by pairs $\left(n_{+}, n_{-}\right)$of nonnegative integers, where $n_{ \pm}=\operatorname{dim}\left(V_{ \pm}\right)$. When $n_{+}$and $n_{-}$have the same parity, the representation $\left(n_{+}, n_{-}\right)$is real and descends to $S O(4)$. Among the representations we shall see quite often are

$$
\begin{gathered}
(2,2) \cong \mathbb{R}^{4}, \\
(3,3) \cong \mathrm{Sym}_{0}^{2},
\end{gathered}
$$

and

$$
(3,1) \cong \bigwedge_{+}^{2}
$$

If we let $\mathbf{n}$ denote the $n$-dimensional irreducible representation of $\mathfrak{s l}(2, \mathbb{C})$, then $\mathbf{n} \otimes \mathbf{m}=\bigoplus_{k=|n-m|+1}^{n+m-1} \mathbf{k}$; using the distributive law we then get the multiplication rule for the representations of $\mathfrak{s o}(4, \mathbb{C})$.

We will let $\left(\mathbb{R}^{4}\right)^{k}$ denote the $k$-fold tensor product of the standard representation, and endow any subspace with the invariant inner product given by taking trace over all $k$ indices. The rational group-ring $\mathbb{Q} S_{k}$ of the symmetric group on $k$ letters acts on $\left(\mathbb{R}^{4}\right)^{k}$, and the kernel and image of the endomorphism of $\left(\mathbb{R}^{4}\right)^{k}$ associated to a given element are invariant subspaces. This remains true if we extend the group-ring by adding in generators $q_{i j}$ which act by contracting the $i$ th and $j$ th factors and then tensoring with the (dual of the) inner product in these factors. Any representation $V$ that arises this way is 'symmetric' in the sense that for any $n, m$, the multiplicities of $(n, m)$ and $(m, n)$ in $V$ are equal. (This can be proven quickly from the fact the the standard representation of $\operatorname{Spin}(4)=S U(2) \times S U(2)$ on $\mathbb{R}^{4}$ is given by leftand right- multiplication by unit quaternions.)

We will be decomposing quite a few tensor representations and identifying copies of irreducible representations within them. When we do so, we will 
simply assert the truth of our decompositions, which are often not immediately obvious, but which follow from basic arguments such as dimension-counting and the symmetry principle above. Similarly we leave to the reader tasks such as verifying the formulas we will give for numerous orthogonal projections, and the fact that all the linear maps we define between representations are $S O(4)$-equivariant.

Our first step is to use the maps $\iota_{k, m}$ (see (6.5)) to recast the system (7.17.4) in terms of representations of $S O(4)$. To avoid introducing still more notation, we will simply regard $\mathcal{H}_{\mathbf{b}}, T, S$, and $U$ above as elements of

$$
\begin{aligned}
& \mathcal{H}=\operatorname{Sym}_{0}^{2} \otimes \mathbb{R}^{4}, \\
& \mathcal{T}=\operatorname{Sym}_{0}^{2} \otimes \mathrm{Sym}^{3}, \\
& \mathcal{S}=\operatorname{Sym}_{0}^{2} \otimes \mathbb{R}^{4} \otimes \mathrm{Sym}^{4},
\end{aligned}
$$

and

$$
\mathcal{U}=\mathrm{Sym}_{0}^{2} \otimes \mathbb{R}^{4} \otimes \mathbb{R}^{4} \otimes \mathrm{Sym}^{5}
$$

respectively. Note that these are tensors of rank 3,5,7, and 9; below, components of tensors of rank $k$ will always be written relative to the standard basis of $\left(\mathbb{R}^{4}\right)^{k}$. Up to sign and irrelevant combinatorial factors we will ignore, $\Delta$, applied to $T, S$ or $U$, is simply trace on the last two indices. Similarly, the map $\partial: \operatorname{Sym}_{0}^{2} \otimes\left(\mathbb{R}^{4}\right)^{k} \mathrm{Sym}^{m} \rightarrow \mathrm{Sym}_{0}^{2} \otimes\left(\mathbb{R}^{4}\right)^{k+1} \otimes \mathrm{Sym}^{m-1}$ is simply the identity tensored with the natural embedding $\operatorname{Sym}^{m} \hookrightarrow \mathbb{R}^{4} \otimes \operatorname{Sym}^{m-1}$.

Let $H_{a b i}$ be the components of $\mathcal{H}_{\mathbf{b}}$, and consider the rank-5 tensor $\Delta^{-1}(H)$ defined by

$$
\left(\Delta^{-1}(H)\right)_{a b i j k}=H_{a b(i} \delta_{j k)} \stackrel{\text { def }}{=} H_{a b i} \delta_{j k}+(i j k) \stackrel{\text { def }}{=} H_{a b i} \delta_{j k}+H_{a b j} \delta_{i k}+H_{a b k} \delta_{i j} .
$$

(Throughout this section we will follow a similar convention for symmetrizing any tensor; see the caption for Table I in the appendices.) This tensor lies in $\mathrm{Sym}_{0}^{2} \otimes \mathrm{Sym}^{3}$ and satisfies (7.1) up to a combinatoric factor $c_{1}^{-1}$. The general solution of (7.1) is therefore given by

$$
T=c_{1} \Delta^{-1}(H)+T^{\prime}
$$


where $T^{\prime}$ represents the general element of the kernel of the map

$$
\Delta: \mathrm{Sym}_{0}^{2} \otimes \mathrm{Sym}^{3} \rightarrow \mathrm{Sym}_{0}^{2} \otimes \mathbb{R}^{4} .
$$

Since $\Delta$ here is the identity on $\operatorname{Sym}_{0}^{2}$ tensored with the trace map from $\mathrm{Sym}^{3}$ to $\mathbb{R}^{4}$, this is equivalent to

$$
T^{\prime} \in \mathcal{T}^{\prime}=\operatorname{Sym}_{0}^{2} \otimes \operatorname{Sym}_{0}^{3},
$$

where $\mathrm{Sym}_{0}^{3}$ is the space of rank-three symmetric traceless tensors.

The solution (7.5) is actually more general than we need, because $\mathcal{H}_{\mathbf{b}}$ is not an arbitrary element of $\mathrm{Sym}_{0}^{2} \otimes \mathbb{R}^{4}$, but rather is given by

$$
H_{b c i}=\frac{1}{6} b^{\mu}\left[2\left(R_{\mu b c i}+R_{\mu c b i}\right)+R_{\mu i} \delta_{b c}\right]
$$

where $\left\{b^{\mu}\right\}$ are the components of $\mathbf{b}$ (see (6.2)). More specifically,

$$
\operatorname{Sym}_{0}^{2} \otimes \mathbb{R}^{4} \cong(3,3) \otimes(2,2) \cong(4,4) \oplus(4,2) \oplus(2,4) \oplus(2,2),
$$

but because of the symmetries of the Riemmann tensor, the $(4,4)$-component (Sym $0_{0}^{3}$-component) of $\mathcal{H}_{\mathrm{b}}$ is zero. (See Table 1 for the projection $\pi_{(4,4)}^{\prime}$ : $\operatorname{Sym}_{0}^{2} \otimes \mathbb{R}^{4} \rightarrow \operatorname{Sym}_{0}^{3}$.) Thus

$$
\mathcal{H}_{\mathbf{b}} \in(4,2) \oplus(2,4) \oplus(2,2) .
$$

It will also be useful for us to identify the three irreducible subspaces in (7.6). The subspace $(2,2)$ is most easily viewed as the image of the embedding

$$
\begin{aligned}
\mathbb{R}^{4} & \hookrightarrow \mathrm{Sym}_{0}^{2} \otimes \mathbb{R}^{4} \\
v & \mapsto X,
\end{aligned}
$$

where

The subspace

$$
X_{a b i}=v_{a} \delta_{b i}+v_{b} \delta_{a i}-\frac{1}{2} v_{i} \delta_{a b}
$$

$$
V_{4224} \stackrel{\text { def }}{=}(4,2) \oplus(2,4)
$$

is characterized by

$$
V_{4224}=\left\{D \in \operatorname{Sym}_{0}^{2} \otimes \mathbb{R}^{4} \mid D_{a b i}+(a b i)=0\right\} ;
$$

note that an element of $V_{4224}$ is automatically traceless. To separate the two irreducible pieces of $V_{4224}$ we use the following observation. 
Lemma 7.1. Let $n \neq m$ and let $V, V^{\prime}$ be representations of $S O(4)$, with $V \cong$ $(n, m) \oplus(m, n)$. Suppose that $(n, m)$ occurs in $\bigwedge_{+}^{2} \otimes V^{\prime}$ but not in $\bigwedge_{-}^{2} \otimes V^{\prime}$, that $(m, n)$ occurs in $\bigwedge_{-}^{2} \otimes V^{\prime}$ but not in $\Lambda_{+}^{2} \otimes V^{\prime}$, and that $w$ is an equivariant isomorphism

$$
V \stackrel{w}{\rightarrow}\left\{(n, m) \oplus(m, n) \subset \bigwedge^{2} \otimes V^{\prime}\right\}
$$

Then the projection $\pi_{n, m}$ from $V$ to the subspace $(n, m)$ (relative to the splitting of $V$ as $(n, m) \oplus(n, m))$ is given by

$$
\pi_{n, m}=w^{-1} \circ\left(p_{+} \otimes 1\right) \circ w
$$

where $p_{+}: \bigwedge^{2} \rightarrow \bigwedge_{+}^{2}$ is the usual self-dual projection.

Proof. Trivial.

Alternatively, one can project using an operator of the form $a \kappa_{n}^{2}+b \kappa_{n}$, where $\kappa_{n}$ is the quadratic Casimir operator of the $n$-dimensional representation of $\mathfrak{s o}(3)_{+}$. For our purposes, however, this is computationally prohibitive.

One of our eventual applications of this lemma will use the subspace $(5,1) \oplus$ $(1,5) \subset \operatorname{Sym}^{2}\left(\bigwedge^{2}\right)$, which is familiar as the 'space of Weyl tensors' that arises in the decomposition of the Riemann tensor into irreducible components. For this reason we refer to maps $w$ as in the lemma as 'Weyl maps'; Table 2 in the appendices lists all the Weyl maps we will need. Our present application of this lemma is to $V_{4224}$. We observe that $\bigwedge_{+}^{2} \otimes \mathbb{R}^{4} \cong(3,1) \otimes(2,2) \cong(4,2) \oplus(2,2)$, which contains $(4,2)$ but not $(2,4)$. The projection from $V_{4224}$ to its $(4,2)$ subspace is therefore computable from the relevant Weyl map $w$ in Table 2. The image of $w$ is the subspace of traceless tensors satisfying the 'Bianchi identity'; i.e. in

$$
\mathcal{F}^{\prime}=\left\{X \in \bigwedge^{2} \otimes \mathbb{R}^{4} \mid X_{a i i}=0 \text { and } X_{a b i}+(a b i)=0\right\} .
$$

From this we find that the (4,2)-piece of $\mathcal{H}_{\mathrm{b}}$ is proportional to $w^{-1} \circ p_{+}(X)$, where $X_{a b k}=a^{\mu}\left[3 R_{\mu k a b}+\delta_{a k} R_{\mu b}-\delta_{b k} R_{\mu a}\right]=a^{\mu}\left[3 W_{\mu k a b}+\frac{1}{2}\left(3 \delta_{\mu a} R_{k b}^{0}-3 \delta_{\mu b} R_{k a}^{0}-\right.\right.$ $\left.\left.\frac{1}{2} \delta_{k a} R_{\mu b}^{0}+\frac{1}{2} \delta_{k b} R_{\mu a}^{0}\right)\right]$; here $W$ is the Weyl curvature tensor and $R^{0}$ the traceless Ricci tensor. This piece vanishes identically if $(M, g)$ is Einstein and anti-selfdual; we will see later why this accounts for the second assertion in Theorem 6.1. (Of course the $(2,4)$-piece of $\mathcal{H}_{\mathbf{b}}$ vanishes under similar conditions, and 
the $(2,2)$-piece vanishes if $(M, g)$ is Ricci-flat, but neither of these cases leads to a simplification of (6.6).) However, the vanishing of the (4,2)-piece of $\mathcal{H}_{\mathrm{b}}$ is not a natural condition geometrically for the applications of Theorem 6.1 we have in mind; $\mathbb{C P}^{2}$, for example, is self-dual.

Now let us return to solving (7.1-7.4). Since (7.5) gives the complete solution of (7.1), we turn next to (7.2). Note that for any connection that is Yang-Mills with respect to the flat metric $\delta$ induced by normal coordinates on $B,\left.(\nabla F)\right|_{B}$ lies in the space $\mathcal{F}^{\prime} \otimes \Gamma\left(\operatorname{End}\left(\left.E\right|_{B}\right)\right)$, after the normal-coordinate identification $T_{x}^{*} M \cong \mathbb{R}^{4}$ is made for each $x \in B$. For a connection that is self-dual with respect to $\delta, \nabla F$ lies in $\mathcal{F}_{(4,2)}^{\prime} \otimes \Gamma\left(\operatorname{End}\left(\left.E\right|_{B}\right)\right)$, where the first factor denotes the $(4,2)$-subspace of $\mathcal{F}^{\prime}$. Now the connections we are interested in are not $\delta$-selfdual, but $g$-self-dual. However, since the difference between the two metrics is $O\left(r^{2}\right)$, the curvature $F$ of a $g$-self-dual connection may be written as the sum of a $\delta$-self-dual two-form plus a term of size $O\left(r^{2}|F|\right)$. Similarly, in (7.27.4) $\nabla F$ is the sum of an element of $\mathcal{F}_{(4,2)}^{\prime} \otimes \Gamma\left(\operatorname{End}\left(\left.E\right|_{B}\right)\right)$ plus a term of size $O\left(r^{2}|\nabla F|\right)$, and so on for higher derivatives. Thus the errors introduced into the explicit terms on the right-hand sides of $(6.6-6.7)$ by replacing $\nabla F$ by its projection to $\mathcal{F}_{(4,2)}^{\prime} \otimes \Gamma\left(\operatorname{End}\left(\left.E\right|_{B}\right)\right)$, with similar replacements for the higher derivatives, are already bounded by the remainder terms in those equations, so it is without loss of generality that we make these replacements henceforth. We will also cease to write the essentially irrelevant factor $\Gamma\left(\operatorname{End}\left(\left.E\right|_{B}\right)\right)$ explicitly.

From the fundamental property of the pairing $\natural$, we have $-\Delta S+\partial T \natural \nabla F \in$ $(1,3) \otimes$ Sym $^{2}$. Since $\nabla F \in \mathcal{F}_{(4,2)}^{\prime}$, we may regard the pairing in $(7.2)$, with a fixed first argument, as giving us an equivariant map from $(4,2)$ to $(1,3) \otimes$ $\mathrm{Sym}^{2}$; moreover we know that $-\Delta S+\partial T \in \mathrm{Sym}_{0}^{2} \otimes \mathbb{R}^{4} \otimes \mathrm{Sym}^{2}$. To solve (7.2) simultaneously for all self-dual connections, then, it is sufficient that any equivariant map from $\operatorname{Sym}_{0}^{2} \otimes \mathbb{R}^{4} \cong(4,4) \oplus(4,2) \oplus(2,4) \oplus(2,2)$ to $\operatorname{Hom}((4,2),(1,3)) \cong(4,2) \otimes(1,3) \cong(4,4) \oplus(4,2)$, when tensored with the identity map of $\mathrm{Sym}^{2}$, vanish on $-\Delta S+\partial T$. Equivalently, we require that the component of $-\Delta S+\partial T$ lying in $[(4,4) \oplus(4,2)] \otimes S^{2}{ }^{2}$ vanish, implying $-\Delta S+\partial T \in[(2,4) \oplus(2,2)] \otimes \mathrm{Sym}^{2}$. Since $\mathrm{Sym}^{2} \cong \mathrm{Sym}_{0}^{2} \oplus \mathbb{R}$, and since $T$ 
satisfies (7.5), this can be expressed as

$$
\Delta S=c_{1} \Delta^{-1}(H)+T^{\prime}+C+\hat{B}
$$

where $\hat{B}_{a b i j k}=B_{a b i} \delta_{j k}$, and where

$$
\begin{aligned}
C \in \mathcal{C}=\{[(2,4) \oplus(2,2)] & \left.\subset \mathrm{Sym}_{0}^{2} \otimes \mathbb{R}^{4}\right\} \otimes \mathrm{Sym}_{0}^{2} \\
B \in \mathcal{B}=[(2,4) \oplus(2,2)] & \subset \mathrm{Sym}_{0}^{2} \otimes \mathbb{R}^{4} .
\end{aligned}
$$

For $T \in \mathrm{Sym}_{0}^{2} \otimes \mathbb{R}^{4} \otimes \mathrm{Sym}^{2}$ (which applies to $T^{\prime}, C$, and $\hat{B}$ ), define

$$
\left(\Delta^{-1}(T)\right)_{a b i j k l m}=T_{a b(i j k} \delta_{l m)}
$$

also define

$$
\left(\Delta^{-1} \Delta^{-1}(H)\right)_{a b i j k l m}=H_{a b(i} \delta_{j k} \delta_{l m)} .
$$

Then the general solution of (7.7) is given by

$$
S=c_{2} \Delta^{-1} \Delta^{-1}(H)+c_{3} \Delta^{-1}\left(T^{\prime}+C\right)+c_{4} \Delta^{-1}(\hat{B})+S^{\prime}
$$

where the $c_{i}$ are easily-computed combinatoric factors and

$$
S^{\prime} \in \operatorname{ker} \Delta: \operatorname{Sym}_{0}^{2} \otimes \mathbb{R}^{4} \otimes \operatorname{Sym}^{4} \rightarrow \operatorname{Sym}_{0}^{2} \otimes \mathbb{R}^{4} \otimes \operatorname{Sym}^{2} .
$$

Since the kernel of the trace map from $\mathrm{Sym}^{4}$ to $\mathrm{Sym}^{2}$ is the space $\mathrm{Sym}_{0}^{4}$, $S^{\prime} \in \operatorname{ker} \Delta$ if and only if

$$
S^{\prime} \in \mathcal{S}^{\prime}=\operatorname{Sym}_{0}^{2} \otimes \mathbb{R}^{4} \otimes \operatorname{Sym}_{0}^{4} .
$$

With the general solution of (7.1-7.2) in hand, we turn to (7.3). We will find the following notation quite useful.

$$
\begin{aligned}
\mathcal{F} & =(3,1) \\
\nabla \mathcal{F} & =\mathcal{F}_{(4,2)}^{\prime}=(4,2) \subset \mathcal{F} \otimes \mathbb{R}^{4} \\
\mathcal{F}^{\prime \prime} & =\mathcal{F} \otimes \mathbb{R}^{4} \otimes \mathbb{R}^{4} \\
\nabla \nabla \mathcal{F} & =\nabla \mathcal{F} \otimes \mathbb{R}^{4} \\
\mathcal{F}^{\prime \prime \prime} & =\mathcal{F} \otimes \mathbb{R}^{4} \otimes \mathbb{R}^{4} \otimes \mathbb{R}^{4} \\
\nabla \nabla \nabla \mathcal{F} & =\nabla \nabla \mathcal{F} \otimes \mathbb{R}^{4}
\end{aligned}
$$

As the notation suggests, given a self-dual connection, the functor assigning to each representation of $S O(4)$ a tensor bundle over $(M, g)$ naturally associates 
subrepresentations of the above spaces with components of the corresponding derivatives of the curvature $F$.

Consider the decomposition $\mathcal{F}^{\prime \prime}=\mathcal{F} \otimes \operatorname{Sym}^{2} \oplus \mathcal{F} \otimes \bigwedge^{2}$. In the corresponding decomposition of $\nabla \nabla F$, the second piece is $K_{2}(F)$, where $K_{2}$ is the curvature of the bundle $E \otimes \wedge^{2} T^{*} M$. Since $K_{2}=F \otimes 1 \oplus 1 \otimes \mathcal{R}$, we have $K_{2}(F)=$ $O\left(|F|+|F|^{2}\right)$. Similarly, the second term in the decomposition $\mathcal{F} \otimes \mathrm{Sym}^{2} \cong$ $\mathcal{F} \otimes \operatorname{Sym}_{0}^{2} \oplus \mathcal{F} \otimes \mathbb{R}$, corresponds to $\Delta F$, which by (3.6) is also $O\left(|F|+|F|^{2}\right)$. Since $\frac{1}{2} \Delta U-\partial S$ is $O\left(r^{3}(|U|+|S|)\right)$, the contributions of both of these terms to the left-hand side of (7.3) are 'harmless' in the sense defined earlier. Thus it suffices to prove (7.3) with $\nabla \nabla F$ replaced by $\left(\operatorname{Sym}_{0}^{2} \nabla\right) F$-i.e., the component of $\nabla \nabla F$ corresponding to $\mathcal{F} \otimes \mathrm{Sym}_{0}^{2}$-or, equivalently, with $\frac{1}{2} \Delta U-\partial S \in$ $\mathrm{Sym}_{0}^{2} \otimes \mathbb{R}^{4} \otimes \mathbb{R}^{4} \otimes \mathrm{Sym}^{3}$ replaced by its projection to $\mathrm{Sym}_{0}^{2} \otimes \mathrm{Sym}_{0}^{2} \otimes \mathrm{Sym}^{3}$.

To make further headway it we consider the decomposition $\nabla \nabla \mathcal{F}$ and $\mathcal{F}^{\prime \prime}$ into irreducible components. From the tensor product rule, we have

$$
\nabla \nabla \mathcal{F}=\mathcal{F}_{(5,3)}^{\prime \prime} \oplus \mathcal{F}_{(5,1)}^{\prime \prime} \oplus\left[\text { some } \mathcal{F}_{(3,3)}^{\prime \prime}\right] \oplus\left[\text { some } \mathcal{F}_{(3,1)}^{\prime \prime}\right]
$$

where $\mathcal{F}_{(n, m)}^{\prime \prime}$ denotes a subrepresentation of $\mathcal{F}^{\prime \prime}$ isomorphic to $(n, m)$; in the cases $(3,3)$ and $(3,1)$, these subrepresentations are not unique, having multiplicity two in $\mathcal{F}^{\prime \prime}$. On the other hand, from the decompositions of $(3,1) \otimes(3,3)$ and $(3,1) \otimes[(3,1) \oplus(1,3)]$ we also have $\mathcal{F} \otimes \operatorname{Sym}_{0}^{2}=\mathcal{F}_{(5,3)}^{\prime \prime} \oplus\left[\right.$ some $\left.\mathcal{F}_{(3,3)}^{\prime \prime}\right] \oplus \mathcal{F}_{(1,3)}^{\prime \prime}$ and $\mathcal{F} \otimes \bigwedge^{2}=\mathcal{F}_{(5,1)}^{\prime \prime} \oplus\left[\right.$ some $\left.\mathcal{F}_{(3,1)}^{\prime \prime}\right] \oplus \mathcal{F}_{(1,1)}^{\prime \prime} \oplus\left[\right.$ some $\left.\mathcal{F}_{(3,3)}^{\prime \prime}\right]$. If we define projections $\pi_{\text {sym }}, \pi_{\text {anti }}$ as the compositions

$$
\begin{gathered}
\nabla \nabla \mathcal{F} \hookrightarrow \mathcal{F}^{\prime \prime} \rightarrow \mathcal{F} \otimes \mathrm{Sym}_{0}^{2} \\
\nabla \nabla \mathcal{F} \hookrightarrow \mathcal{F}^{\prime \prime} \rightarrow \mathcal{F} \otimes \bigwedge^{2}
\end{gathered}
$$

respectively, where the last arrow in each case is the obvious projection, it follows that that image $\left(\pi_{\text {sym }}\right) \subset \mathcal{F}_{(5,3)}^{\prime \prime} \oplus\left[\right.$ some $\left.\mathcal{F}_{(3,3)}^{\prime \prime}\right]$ and that image $\left(\pi_{\text {anti }}\right) \subset$ $\mathcal{F}_{(5,1)}^{\prime \prime} \oplus\left[\right.$ some $\left.\mathcal{F}_{(3,3)}^{\prime \prime}\right] \oplus\left[\right.$ some $\left.\mathcal{F}_{(3,1)}^{\prime \prime}\right]$, since distinct representations cannot intertwine. Now any element of $\nabla \nabla \mathcal{F}$ can be written as the sum of its symmetric and antisymmetric parts, so by looking at the decomposition of $\nabla \nabla \mathcal{F}$ we see that the four distinct representations appearing in the (possible) images of these two projections must in fact be present; the only question is whether $(3,3)$ appears in both images, and in fact it does. To prove that it appears 
in image $\left(\pi_{\text {anti }}\right)$, for example, we can define a map $j: \operatorname{Sym}_{0}^{2} \rightarrow \Lambda^{2} \otimes \Lambda^{2}$ by $j(X)_{i j k l}=\delta_{i k} X_{j l}-\delta_{i l} X_{j k}-\delta_{j k} X_{i l}+\delta_{j l} X_{i k}$ and observe that the composition

$$
\mathrm{Sym}_{0}^{2} \stackrel{j}{\hookrightarrow} \bigwedge^{2} \otimes \bigwedge^{2} \stackrel{p_{+} \otimes 1}{\longrightarrow} \mathcal{F} \otimes \bigwedge^{2} \stackrel{t r_{13}}{\longrightarrow} \mathrm{Sym}_{0}^{2}
$$

is a nonzero multiple of the identity. (Here $t r_{13}$ denotes trace between the first and third indices of $\Lambda^{2} \otimes \Lambda^{2}$.) By changing two of the signs in the definition of $j$ we prove similarly that image $\left(\pi_{s y m}\right) \supset(3,3)$. By Schur's lemma the map

$$
k:(3,3) \hookrightarrow \operatorname{image}\left(\pi_{\text {anti }}\right) \hookrightarrow \nabla \nabla \mathcal{F} \stackrel{\pi_{\text {sym }}}{\longrightarrow} \operatorname{image}\left(\pi_{\text {sym }}\right)
$$

is either zero or an isomorphism; if zero then $\nabla \nabla \mathcal{F}$ would contain two independent copies of $(3,3)$, which it does not. Hence $k$ is an isomorphism. As a consequence, the $(3,3)$-component of $\left(\operatorname{Sym}_{0}^{2} \nabla\right) F$ can be expressed linearly in terms of $\left(\bigwedge^{2} \nabla\right) F$, so $\left|\left(\operatorname{Sym}_{0}^{2} \nabla F\right)_{(3,3)}\right| \leq c\left|K_{2}(F)\right|$, and as before we see that the contribution of this component to the left-hand side of (7.3) is harmless. Thus we can further replace $\frac{1}{2} \Delta U-\partial S$ in (7.3) by its projection to $\left[(5,3) \subset \mathrm{Sym}_{0}^{2} \otimes \mathrm{Sym}_{0}^{2}\right] \otimes \mathrm{Sym}^{3}$, which we denote by $\left(\frac{1}{2} \Delta U-\partial S\right)_{(5,3) \otimes \mathrm{Sym}^{3}}$. (As the reader can check, $\operatorname{Sym}_{0}^{2} \otimes \operatorname{Sym}_{0}^{2}$ contains a unique copy of $(5,3)$.)

To solve (7.3), then, it suffices to set equal to zero any component of $\left(\frac{1}{2} \Delta U+\partial S\right)_{(5,3) \otimes \text { Sym }^{3}}$ lying in a subrepresentation isomorphic to an irreducible component of

$$
\operatorname{Hom}((5,3),(1,3)) \otimes \mathrm{Sym}^{3} \cong[(5,5) \oplus(5,3) \oplus(5,1)] \otimes \mathrm{Sym}^{3} .
$$

Letting $\pi_{(5, m)}, m=1,2,3$, denote the projection from $\mathrm{Sym}_{0}^{2} \otimes \mathrm{Sym}_{0}^{2}$ to the subspace $(5, m)$ (each of which has multiplicity 1 ), letting $\pi_{S}$ denote the projection $1 \otimes \pi_{\mathrm{Sym}_{0}^{2}} \otimes 1: \mathrm{Sym}_{0}^{2} \otimes \mathbb{R}^{4} \otimes \mathbb{R}^{4} \otimes \mathrm{Sym}^{3}$ (see Table 1 ), and using (7.8), we see that it suffices to solve the system

$$
\left\{\begin{array}{l}
\left(\pi_{(5,5)} \otimes 1\right)\left(\pi_{S}(X)\right)=\left(\pi_{(5,5)} \otimes 1\right)\left(\pi_{S}\left(\Delta^{-1} \Delta^{-1} H\right)\right) \\
\left(\pi_{(5,3)} \otimes 1\right)\left(\pi_{S}(X)\right)=\left(\pi_{(5,3)} \otimes 1\right)\left(\pi_{S}\left(\Delta^{-1} \Delta^{-1} H\right)\right) \\
\left(\pi_{(5,1)} \otimes 1\right)\left(\pi_{S}(X)\right)=\left(\pi_{(5,1)} \otimes 1\right)\left(\pi_{S}\left(\Delta^{-1} \Delta^{-1} H\right)\right)
\end{array}\right.
$$

where $X=\Delta U+\Delta^{-1}\left(T^{\prime}+C+B\right)+S^{\prime}$ and $T^{\prime}, C, B$ and $S^{\prime}$ are the tensors appearing in (7.8), redefined to absorb some constants. 
A similar but longer argument applies to (7.4). We start by considering the decomposition $\mathbb{R}^{4} \otimes \mathbb{R}^{4} \otimes \mathbb{R}^{4} \cong \operatorname{Sym}^{3} \oplus\left[\mathbb{R}^{4} \otimes \Lambda^{2}+\Lambda^{2} \otimes \mathbb{R}^{4}\right]$. The sum in the second factor is not direct; the intersection of the two summands is $\Lambda^{3}$. Tensoring through with $\mathcal{F}$ we find that $\left(1 \otimes \pi_{\mathbb{R}^{4} \otimes \Lambda^{2}}\right)(\nabla \nabla \nabla \mathcal{F}) \cong \nabla \mathcal{F} \otimes \bigwedge^{2}$, which contains $(4,4)$ exactly once but does not contain $(2,4)$, that $\left(1 \otimes \pi \Lambda^{2} \otimes \mathbb{R}^{4}\right)(\nabla \nabla \nabla \mathcal{F}) \cong$ $\left(\bigwedge^{2} \nabla\right) \mathcal{F} \otimes \mathbb{R}^{4}$, which contains $(4,4)$ and $(2,4)$ exactly once each, and that the intersection $\left(1 \otimes \pi \Lambda^{3}\right)(\nabla \nabla \nabla \mathcal{F}) \subset \mathcal{F} \otimes \Lambda^{3} \cong(3,1) \otimes(2,2)$ does not contain

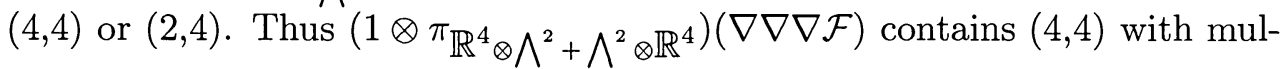
tiplicity two and $(2,4)$ with multiplicity one. These representations have the

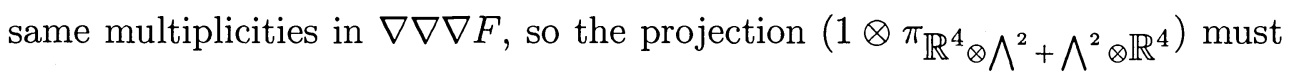
be an isomorphism on these subspaces. Now $\mathrm{Sym}^{3}=\mathrm{Sym}_{0}^{3} \oplus$ (complement), and it is not hard to check that the complement lies in the (non-direct) sum $\left[\mathbb{R} \subset \mathrm{Sym}^{2}\right] \otimes \mathbb{R}^{4}+\mathbb{R}^{4} \otimes \Lambda^{2}+\bigwedge^{2} \otimes \mathbb{R}^{4}$. Tensoring through with $\mathcal{F}$ and reinterpreting this decomposition in terms of the derivatives of a curvature two-form $F$, we find that the first term in the complement corresponds to $\nabla(\Delta F)$, the second to $K_{3}(\nabla F)$ (where $K_{3}$ is the curvature of $\left.E \otimes\left(T^{*} M\right)^{3}\right)$, and the third to $\nabla\left(K_{2}(F)\right)$. Using Lemma 3.1 and (3.6), we see that all of these give rise to 'harmless' terms in (7.4), so the only potentially 'harmful' terms are those coming from $\mathcal{F} \otimes \mathrm{Sym}_{0}^{3}$. But this space is isomorphic to $(6,4) \oplus(4,4) \oplus(2,4)$, and we have seen above that all the $(4,4)$ - and $(2,4)$-components of $\nabla \nabla \nabla \mathcal{F}$ project isomorphically to the 'harmless' sub-

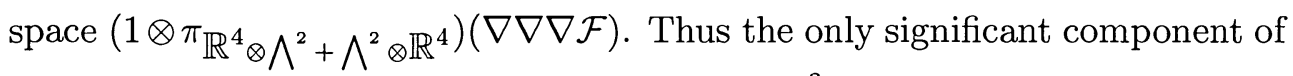
$\nabla \nabla \nabla F$ in (7.4) is the $(6,4)$-component of $\left(\operatorname{Sym}_{0}^{3} \nabla\right) F$, and we need only set equal to zero any component of $(\partial U)_{\left[(6,4) \subset \mathrm{Sym}_{0}^{2} \otimes \mathrm{Sym}_{0}^{3}\right] \otimes \mathrm{Sym}^{4}}$ lying in a subrepresentation isomorphic to an irreducible component of

$$
\operatorname{Hom}((6,4),(1,3)) \otimes \mathrm{Sym}^{4} \cong[(6,6) \oplus(6,4) \oplus(6,2)] \otimes \mathrm{Sym}^{4} .
$$

This can be re-expressed as a system of equations of the form

$$
\left\{\begin{array}{l}
\left(\pi_{(6,6)} \otimes 1\right)\left(\pi_{U}(X)\right)=\left(\pi_{(6,6)} \otimes 1\right)\left(\pi_{U}\left(\Delta^{-1} \Delta^{-1} \Delta^{-1} H\right)\right) \\
\left(\pi_{(6,4)} \otimes 1\right)\left(\pi_{U}(X)\right)=\left(\pi_{(6,4)} \otimes 1\right)\left(\pi_{U}\left(\Delta^{-1} \Delta^{-1} \Delta^{-1} H\right)\right) \\
\left(\pi_{(6,2)} \otimes 1\right)\left(\pi_{U}(X)\right)=\left(\pi_{(6,2)} \otimes 1\right)\left(\pi_{U}\left(\Delta^{-1} \Delta^{-1} \Delta^{-1} H\right)\right)
\end{array}\right.
$$

where $X$ is an appropriate tensor, where $\pi_{U}=1 \otimes \pi_{\mathrm{Sym}_{0}^{3}} \otimes 1: \mathrm{Sym}_{0}^{2} \otimes \mathbb{R}^{4} \otimes$ 
$\mathbb{R}^{4} \otimes \mathbb{R}^{4} \otimes \operatorname{Sym}^{4} \rightarrow \operatorname{Sym}_{0}^{2} \otimes \operatorname{Sym}_{0}^{3} \otimes \operatorname{Sym}^{4}$ (see Table 1 ), and where the meanings of $\pi_{(6, m)}$ should be obvious. (The precise definition of $\Delta^{-1} \Delta^{-1} \Delta^{-1} H$ is irrelevant.)

It is sufficient for us to solve (7.1-7.4) separately in the three cases corresponding to the three irreducible subspaces in (7.6); we will proceed from the easiest case to the hardest. Only in the last case will we need to worry about $U$.

CASE $1 . \mathcal{H}_{\mathrm{b}} \in(2,2)$

We start by setting $U=0$, so that (7.4) is automatically satisfied, and by setting to zero any component of $S$ lying in an irreducible subspace of $\mathcal{S}$ not isomorphic to $(2,2)$. We demand that the $(2,2)$-components be the images of a standard copy of $(2,2)$ under as-yet to be determined equivariant isomorphisms. The spaces $\mathcal{T}^{\prime}, \mathcal{C}, \mathcal{B}$, and $\mathcal{S}^{\prime}$ defined earlier contain $(2,2)$ with multiplicity 1,2,1, and 1 respectively; by Schur's lemma there are thus five free parameters in the general solution (7.8). Completely decomposing (7.10), using $\operatorname{Sym}^{3} \cong \operatorname{Sym}_{0}^{3} \oplus \mathbb{R}^{4} \cong(4,4) \oplus(2,2)$, we find that $(2,2)$ occurs uniquely in each of $(5,5) \otimes \mathrm{Sym}^{3}$ and $(5,3) \otimes \mathrm{Sym}^{3}$ and is absent from $(5,1) \otimes \mathrm{Sym}^{3}$; thus $(2,2)$ occurs with multiplicity two in (7.10). If we define an equivariant isomorphism from the direct sum of these two copies of $(2,2)$ to a standard copy of $2(2,2)$, and compose with the maps $\pi \otimes 1$ on the left-hand side of (7.11), we obtain a map $\oplus_{1}^{5}(2,2) \rightarrow \oplus_{1}^{2}(2,2)$, or equivalently from $\mathbb{R}^{5} \otimes(2,2) \rightarrow$ $\mathbb{R}^{2} \otimes(2,2)$, where $S O(4)$ acts trivially on the first factor in the source and target spaces. By Schur's lemma this map must be of the form $\Pi \otimes 1$, where $\Pi=\Pi^{(2,2)}: \mathbb{R}^{5} \rightarrow \mathbb{R}^{2}$. The system (7.11) will be solvable if $\Pi$ is surjective, and a splitting of the exact sequence $0 \rightarrow \mathbb{R}^{3} \rightarrow \mathbb{R}^{5} \stackrel{\Pi}{\rightarrow} \mathbb{R}^{2} \rightarrow 0$ will give a solution that depends linearly on $\mathcal{H}_{\mathrm{b}}$. Thus it suffices to show that $\Pi$ has rank two, which we accomplish by finding a nonsingular $2 \times 2$ submatrix of a matrix representing $\Pi$.

To do this it suffices to ignore $B$ and $S^{\prime}$, and define maps $\mathbb{R}^{4} \rightarrow \mathcal{T}^{\prime}, \mathbb{R}^{4} \rightarrow \mathcal{C}$ by

$T^{\prime}(v)_{i j k l m}=T_{i j k l m}^{\prime}=-\frac{1}{3} v_{(i} \delta_{j)(k} \delta_{l m)}+\left[v_{k}\left(\delta_{i l} \delta_{j m}+\delta_{i m} \delta_{j l}-\frac{1}{3} \delta_{i j} \delta_{l m}+(k l m)\right]\right.$, 


$$
C(v)_{i j k l m}=C_{i j k l m}=\left[v_{k}\left(\delta_{i l} \delta_{j m}+\delta_{i m} \delta_{j l}-\frac{1}{2} \delta_{i j} \delta_{l m}+(k l m)\right],\right.
$$

where $v \in \mathbb{R}^{4}$. To obtain an isomorphism from the 2(2,2)-subspace of (7.10) to $\mathbb{R}^{4} \oplus \mathbb{R}^{4}$, we use the map $\operatorname{Tr}^{(2,2)}=\left(\left(\pi_{(5,5)} \otimes 1\right) \circ \operatorname{tr}_{25,36,47},\left(\pi_{(5,3)} \otimes 1\right) \circ \operatorname{tr}_{25,36,47}\right)$, where

$$
\left(\operatorname{tr}_{25,36,47}(X)\right)_{i}=X_{\text {iabcabc }} .
$$

It is not immediately obvious that $\operatorname{Tr}^{(2,2)}$ is an isomorphism, but since (7.10) contains exactly two copies of $(2,2)$ this will be verified a posteriori when we show that the $2 \times 2$ matrix mentioned above has rank two.

The only remaining ingredients required to compute this matrix are formulas for the projections $\pi_{(5, m)}$. Since $(5,5)$ in this context is the subspace $\mathrm{Sym}_{0}^{4}$ of $\mathrm{Sym}_{0}^{2} \otimes \mathrm{Sym}_{0}^{2}$-in general, $\operatorname{Sym}_{0}^{n} \cong(n+1, n+1)$, and $\mathrm{Sym}^{n} \cong$ $\oplus_{j=0}^{[n / 2]}(n+1-2 j, n+1-2 j)$-this projection is relatively easy to compute; see table 1 . The projection $\pi_{(5,3)}$ is harder to get one's hands on because $(5,3)$ is not a 'symmetric' representation. By dimension-counting and symmetry, however, it is not hard to show that

$$
\operatorname{Sym}^{2}\left(\operatorname{Sym}_{0}^{2}\right) \cong[(5,5) \oplus(3,3) \oplus(1,1)] \oplus[(5,1) \oplus(1,5)]
$$

and that

$$
\bigwedge^{2}\left(\operatorname{Sym}_{0}^{2}\right) \cong[(5,3) \oplus(3,5)] \oplus[(3,1) \oplus(1,3)] .
$$

By antisymmetrizing and taking traces-the subspaces $(5,3) \oplus(3,5)$ and $(5,1) \oplus$ $(1,5)$ are traceless - it is again not hard to obtain a formula for the projection $\pi_{5335}: \mathrm{Sym}_{0}^{2} \otimes \mathrm{Sym}_{0}^{2} \rightarrow$ subspace isomorphic to $(5,3) \oplus(3,5)$. To project from this subspace down to $(5,3)$, we use the Weyl map $w: \Lambda^{2}\left(\mathrm{Sym}_{0}^{2}\right) \rightarrow \operatorname{Sym}_{0}^{2} \otimes \Lambda^{2}$ given in Table 2, and define a projection $\pi_{53 \leftarrow 5335}$, as in Lemma 7.1. Then $\pi_{(5,3)}=\pi_{53 \leftarrow 5335} \circ \pi_{5335}$.

We can now compute the $2 \times 2$ matrix defined above. These computations are extremely cumbersome-after all, we are dealing with tensors with sevenindex tensors, and Case 3 will involve nine indices-and we leave the details to the reader. (The author left the details to Maple.) In the end, the matrix one computes is

$$
\left(\begin{array}{rr}
50 & 150 \\
-50 & -30
\end{array}\right)
$$


As claimed, this has rank two, and $\Pi^{(2,2)}$ is surjective.

CASE 2. $\mathcal{H}_{\mathbf{b}} \in(2,4)$

The procedure is almost identical to the previous one, but with $(2,2)$ replaced by $(2,4)$. The spaces $\mathcal{T}^{\prime}, C, B$ and $\mathcal{S}^{\prime}$ contain $(2,4)$ with multiplicity $1,2,1$, and 2 respectively, while each of the spaces $(5, m) \otimes \operatorname{Sym}^{3}$ for $m=1,2,3$ contains $(2,4)$ exactly once. Thus we are reduced to showing that a map $\Pi^{(2,4)}: \mathbb{R}^{6} \rightarrow \mathbb{R}^{3}$, defined analogously to $\Pi^{(2,2)}$, is surjective. We take our standard copy of $(2,4)$ to be the one contained in $V_{4224} \subset \mathrm{Sym}_{0}^{2} \otimes \mathbb{R}^{4}$; using Lemma 7.1 with orientations reversed, the $(2,4)$ - subspace can be explicitly identified using the isomorphism $V_{4224} \cong \mathcal{F}^{\prime}$ given in Table 1 . We define a map $\operatorname{tr}_{15,26}: \mathcal{S} \rightarrow \mathbb{R}^{4} \otimes \mathbb{R}^{4} \otimes \mathbb{R}^{4}$ by $\operatorname{tr}_{15,26}(X)_{i j k}=X_{a b i j a b k}$ and set $\operatorname{Tr}^{(2,4)}=\left(\left(\pi_{(5,5)} \otimes 1\right) \circ \operatorname{tr}_{15,26},\left(\pi_{(5,3)} \otimes 1\right) \circ \operatorname{tr}_{15,26},\left(\pi_{(5,1)} \otimes 1\right) \circ \operatorname{tr}_{15,26}\right)$; this restricts to isomorphism from the $3(2,4)$ - subspace of $(7.10)$ to the direct sum of three standard copies. From (7.15) it is not hard to compute the projection $\pi_{5115}$ anälogous to $\pi_{5335}$ (see Table 1 ). Obtaining the projection $\pi_{51 \leftarrow 5115}$ from a Weyl map given in Table 2 , we then have $\pi_{(5,1)}=\pi_{51 \leftarrow 5115} \circ \pi_{5115}$.

Since we only need to show $\Pi^{(2,4)}$ has rank three, we need only find a nonsingular $3 \times 3$ submatrix of the matrix representing this map. For $X \in$ $(2,4)$, we define $T^{\prime}(X), C(X)$, and $S^{\prime}(X)$ by

$$
\begin{gathered}
T_{i j k l m}^{\prime}=X_{i j(k} \delta_{l m)}+3\left(\delta_{i(k} X_{l m) j}+\delta_{j(k} X_{l m) i}\right), \\
C_{i j k l m}=\left[\left(X_{k i l}-X_{k l i}\right) \delta_{j m}+(i j)\right]+(l m),
\end{gathered}
$$

and

$$
\begin{aligned}
S_{a b i j k l m}^{\prime}= & \left\{\left[X_{j k i}\left(\delta_{a l} \delta_{b m}+\delta_{a m} \delta_{b l}-\frac{1}{2} \delta_{a b} \delta_{l m}\right)\right]+(j k l m)\right\} \\
& -\frac{1}{4}\left\{\left[\delta_{j k}\left(\delta_{l(a} X_{b) m i}+(l m)\right)-X_{l m i} \delta_{a b}\right]+(j k l m)\right\} \\
& +\frac{1}{6} X_{a b i} \delta_{(j k} \delta_{l m)} .
\end{aligned}
$$

With respect to these maps, the $3 \times 3$ submatrix of $\Pi^{(2,4)}$ is found to be

$$
\frac{25}{3}\left(\begin{array}{rrr}
-8 & 4 & -1 \\
-4 & 4 & -2 \\
4 & 1 & -1
\end{array}\right)
$$


This has rank 3 , and $\Pi^{(2,4)}$ is surjective.

CASE 3. $\mathcal{H}_{\mathrm{b}} \in(4,2)$

Unfortunately we cannot take $U=0$ in this case. If we do, and proceed as before, we obtain a map $\Pi: \mathbb{R}^{5} \rightarrow \mathbb{R}^{4}$. One has every right to expect a linear map from $\mathbb{R}^{5}$ to $\mathbb{R}^{4}$ to be surjective, but, rather surprisingly, one finds that $\Pi$ only has rank 3 . (Not surprisingly for a map of rank 3 , its image does not contain the image of the right-hand side of the system (7.11), which is a line in $\mathbb{R}^{4}$ ). This seems to be a consequence of low-dimensional crowding that disappears when we allow $U$ to be nonzero. In fact, there is so much room in the space $\mathcal{U}$ that we can find a solution to (7.1-7.4) for which

$$
\frac{1}{2} \Delta U-\partial S=0
$$

rather than the more delicate condition that led to (7.11). The general solution of (7.19), given (7.8), is

$$
U=c_{5} \Delta^{-1} \Delta^{-1} \Delta^{-1}(H)+\Delta^{-1} \Delta^{-1}\left(c_{6}\left(T^{\prime}+C\right)+c_{7} \hat{B}\right)+c_{8} \Delta^{-1}\left(S^{\prime}\right)+U^{\prime},
$$

where $U^{\prime} \in \operatorname{ker} \Delta$, and where we define $\Delta^{-1}: \mathcal{S}^{\prime}+\Delta^{-1}\left(\mathcal{T}^{\prime}+\mathcal{C}\right)$ by

$$
\left(\Delta^{-1}(S)\right)_{a b i j k l m n p}=S_{a b i(j k l m} \delta_{n p)}
$$

(The precise definitions of the other $\Delta^{-1}$ are unimportant.) We will find a solution in which $B$ and $U^{\prime}$ are zero; thus we can take $X=\Delta^{-1} \Delta^{-1}\left(T^{\prime}+C\right)+$ $\Delta^{-1}\left(S^{\prime}\right)$ in $(7.13)$, after some redefinitions to absorb constants.

The space (7.12) contains $(4,2)$ with multiplicity four, with one copy coming from $(6,6) \otimes \operatorname{Sym}^{4}$, two from $(6,4) \otimes \operatorname{Sym}^{4}$, and one from $(6,2) \otimes \mathrm{Sym}^{4}$. Thus it suffices to define four embeddings of $(4,2)$ into $\mathcal{U}$ and show that the appropriately-defined map $\Pi^{(4,2)}: \mathbb{R}^{4} \rightarrow \mathbb{R}^{4}$ is surjective. For the first three embeddings we use the formulas (7.16-7.18), but with $X$ taken from $(4,2) \subset \operatorname{Sym}_{0}^{2} \otimes \mathbb{R}^{4}$ instead of $(2,4)$, and apply $\Delta^{-1}$ as needed. For the fourth embedding we apply $\Delta^{-1}$ to

$$
\begin{aligned}
S_{a b i j k l m}^{\prime \prime}= & \left\{\left[X_{j k a} \delta_{i(l} \delta_{m) b}+(a b)\right]+(j k l m)\right\} \\
& -\frac{1}{4}\left\{\delta_{j k}\left[\left(\left(X_{i l(a} \delta_{b) m}-X_{a b l} \delta_{i m}\right)+(l m)\right)+X_{l m(a} \delta_{b) i}\right]+(j k l m)\right\}
\end{aligned}
$$




$$
-\frac{1}{6} X_{a b i} \delta_{(j k} \delta_{l m)}
$$

The next step is to identify the projections $\pi_{(6, m)}: \mathrm{Sym}_{0}^{2} \otimes \mathrm{Sym}_{0}^{3} \rightarrow[(6, \mathrm{~m})$ subspace] that appear in (7.13); we proceed as in the earlier cases. In this case $(6,6) \cong \operatorname{Sym}_{0}^{5}$ is easy to identify, but the subspaces $(6,4) \oplus(4,6)$ and $(6,2) \oplus(2,6)$ seem to have no simpler description as subspaces of $\mathrm{Sym}_{0}^{2} \otimes \mathrm{Sym}_{0}^{3}$ than that they are the kernels of $1-\pi_{6446}$ and $1-\pi_{6226}$ respectively (see Table 1). (We note in passing, though, that $(6,6) \oplus(6,4) \oplus(4,6) \oplus(6,2) \oplus(2,6)$ is precisely the totally traceless subspace of $\operatorname{Sym}_{0}^{2} \otimes \operatorname{Sym}_{0}^{3}$, just as $(5,5) \oplus$ $(5,3) \oplus(3,5) \oplus(5,1) \oplus(1,5)$ is the totally traceless subspace of $\mathrm{Sym}_{0}^{2} \otimes \mathrm{Sym}_{0}^{2}$.) The images of these spaces under the Weyl maps in Table 2 are easier to understand; $(6,4) \oplus(4,6)$ is identified as the subspace of $\left(\bigwedge^{2} \otimes \operatorname{Sym}_{0}^{3}\right)_{0}$ satisfying the Bianchi identity on the first three indices, while $(6,2) \oplus(2,6) \subset$ (space $\mathcal{W}$ of Weyl tensors ) $\otimes \mathbb{R}^{4} \subset \Lambda^{2} \otimes \Lambda^{2} \otimes \mathbb{R}^{4}$ is just the totally traceless subspace $\left(\mathcal{W} \otimes \mathbb{R}^{4}\right)_{0}$ (equivalently, the subspace of $\mathcal{W} \otimes \mathbb{R}^{4}$ satisyfing the Bianchi identity on the last three indices). For $m=2,4$ the Weyl maps give us projections $\pi_{6 m \leftarrow 6 m m 6}$ and we set $\pi_{(6, m)}=\pi_{6 m \leftarrow 6 m m 6} \circ \pi_{6 m m 6}$.

Finally, we need an isomorphism from the 4(4,2) subspace of (7.12) to the direct sum of four standard copies (each of which we again take to lie in $\left.\operatorname{Sym}_{0}^{2} \otimes \mathbb{R}^{4}\right)$, and for this we make use of the triple traces $\operatorname{tr}_{16,27,38}(X)_{i j k}=$ $X_{a b c i j a b c k}, \operatorname{tr}_{36,47,89}(X)_{i j k}=X_{i j a b k a b c c}$. The map

$$
\operatorname{Tr}^{(4,2)}=\left(\operatorname{tr}_{16,27,38} \circ \pi_{(6,6)}, \operatorname{tr}_{16,27,38} \circ \pi_{(6,4)}, \operatorname{tr}_{36,47,89} \circ \pi_{(6,4)}, \operatorname{tr}_{16,27,38} \circ \pi_{(6,2)}\right)
$$

gives the desired isomorphism. The matrix of $\Pi^{(4,4)}$ is found to be

$$
\left(\begin{array}{rrrr}
-1008 & 1512 & -126 & -252 \\
128 & -432 & -64 & -168 \\
-3200 & 2400 & -80 & 0 \\
400 & -120 & 10 & 36
\end{array}\right)
$$

which has rank four, as desired.

The linear maps $L^{(k)}$ of Theorem 6.1 are simply the sums of $S, T$, and $U$ for the three cases above. This completes the proof of this theorem, and hence of Proposition 5.1 as well. 


\section{LOCALiZATION OF THE CURVATURE FORMULA: FinAL STAGE}

We are ready to estimate both the approximate sectional curvature $\tilde{\sigma}$ and its difference from the true curvature $\sigma$. First we introduce some notation to shorten several long formulas. Throughout this section we abbreviate $\pi_{A}$ as $\pi$, and we use the notation $\xi_{Z}=\pi \tilde{Z}-\tilde{Z}$ as in (5.1). We will frequently encounter remainder terms of the form $\mathrm{R}_{i}(\tilde{Z}, \tilde{W}), \mathrm{R}_{i}\left(\tilde{Z}, \xi_{W}\right)$, and $\mathrm{R}_{i}\left(\tilde{W}, \xi_{Z}\right)$ (see Proposition 2.1), the details of whose arguments will often be largely irrelevant. For that purpose we let $R_{Z W}$ generically denote any constantcoefficient linear combination of such terms; note that this includes $\mathrm{R}_{i}(\tilde{Z}, \pi \tilde{W})$ and $\mathrm{R}_{i}(\tilde{W}, \pi \tilde{Z})$.

Recalling Corollary 2.5, we have

$$
\begin{aligned}
\sigma_{A}(\pi \tilde{Z}, \pi \tilde{W}) & =\tilde{\sigma}_{A}(\tilde{Z}, \tilde{W})+\left(\sigma_{A}(\pi \tilde{Z}, \pi \tilde{W})-\tilde{\sigma}_{A}(\pi \tilde{Z}, \pi \tilde{W})\right) \\
& =\frac{\operatorname{Rem}(Z, W)+\operatorname{num}(\pi \tilde{Z}, \pi \tilde{W} ; A)-\operatorname{num}(\tilde{Z}, \tilde{W} ; A)}{\operatorname{denom}(\pi \tilde{Z}, \pi \tilde{W})}
\end{aligned}
$$

where

$$
\begin{aligned}
\operatorname{Rem}(Z, W)= & \frac{3}{2}\left\langle\iota_{Z} \tilde{W}, \mathrm{R}_{0}(\tilde{Z}, \tilde{W})\right\rangle+3\left\langle\mathrm{R}_{0}(\tilde{Z}, \tilde{W}), G_{0}^{A}\left(\mathrm{R}_{0}(\tilde{Z}, \tilde{W})\right)\right\rangle \\
& -\left\langle\left(Z^{*} \wedge \tilde{W}\right)_{-}, \mathrm{R}_{2}(\tilde{Z}, \tilde{W})\right\rangle+\left\langle\left(W^{*} \wedge \tilde{W}\right)_{-}, \mathrm{R}_{2}(\tilde{Z}, \tilde{Z})\right\rangle \\
& +\left\langle R_{Z W}, G_{-}^{A} R_{Z W}\right\rangle+\left\langle R_{Z Z}, G_{-}^{A} R_{W W}\right\rangle .
\end{aligned}
$$

To write out the difference of the 'num' terms in (8.1) in usable form, we start by noting that

$$
\begin{aligned}
\left.\{\pi \tilde{Z}, \pi \tilde{W}\}, G_{0}^{A}\{\pi \tilde{Z}, \pi \tilde{W}\}\right\rangle-\left\langle\{\tilde{Z}, \tilde{W}\}, G_{0}^{A}\{\tilde{Z}, \tilde{W}\}\right\rangle \\
\quad=\left\langle\{\pi \tilde{Z}, \pi \tilde{W}\}-\{\tilde{Z}, \tilde{W}\}, G_{0}^{A}(\{\pi \tilde{Z}, \pi \tilde{W}\}+\{\tilde{Z}, \tilde{W}\})\right\rangle
\end{aligned}
$$

From (5.1) we have

$$
\{\pi \tilde{Z}, \pi \tilde{W}\}-\{\tilde{Z}, \tilde{W}\}=\left\{\tilde{Z}, \xi_{W}\right\}-\left\{\tilde{W}, \xi_{Z}\right\}+\left\{\xi_{Z}, \xi_{W}\right\}
$$

and

$$
\{\pi \tilde{Z}, \pi \tilde{W}\}+\{\tilde{Z}, \tilde{W}\}=\{\tilde{Z}, \pi \tilde{W}\}-\{\tilde{W}, \pi \tilde{Z}\}+\left\{\xi_{Z}, \xi_{W}\right\} .
$$

Inserting these expressions into (8.3), we can use (2.2) to manipulate the righthand side of (8.3) in the same fashion as in (2.21). After a judicious regrouping 
of terms, using $\iota_{W} \tilde{Z}=-\iota_{Z} \tilde{W}$, we find

$$
\begin{aligned}
& \left.\quad\left\langle\{\pi \tilde{Z}, \pi \tilde{W}\}, G_{0}^{A}\{\pi \tilde{Z}, \pi \tilde{W}\}\right\rangle-\{\tilde{Z}, \tilde{W}\}, G_{0}^{A}\{\tilde{Z}, \tilde{W}\}\right\rangle \\
& =\frac{1}{2}\left\{\left\langle\left\{\xi_{Z}, \pi \tilde{W}\right\}, \iota_{Z} \pi \tilde{W}\right\rangle+\left\langle\left\{\xi_{Z}, \tilde{W}\right\}, \iota_{Z} \tilde{W}\right\rangle+\left\langle\left\{\pi \tilde{W}, \xi_{Z}\right\}, \iota_{W} \xi_{Z}\right\rangle+(W \leftrightarrow Z)\right\} \\
& \quad+\frac{1}{2}\left\langle\iota_{Z} \xi_{W}-\iota_{W} \xi_{Z}, \mathrm{R}_{0}(\tilde{Z}, \pi \tilde{W})-\mathrm{R}_{0}(\tilde{W}, \pi \tilde{Z})\right\rangle \\
& \quad+\left\langle\mathrm{R}_{0}(\tilde{Z}, \pi \tilde{W})-\mathrm{R}_{0}(\tilde{W}, \pi \tilde{Z}), G_{0}^{A}\left(\mathrm{R}_{0}\left(\tilde{Z}, \xi_{W}\right)-\mathrm{R}_{0}\left(\tilde{W}, \xi_{Z}\right)\right)\right\rangle \\
& \quad+\left\langle R_{Z W}, G_{0}^{A}\left\{\xi_{Z}, \xi_{W}\right\}\right\rangle+\left\langle\left\{\xi_{Z}, \xi_{W}\right\}, G_{0}^{A}\left\{\xi_{Z}, \xi_{W}\right\}\right\rangle .
\end{aligned}
$$

(Here $(W \leftrightarrow Z)$ means the preceding set of terms with $W$ and $Z$ interchanged.) Similarly, using $\left(W^{*} \wedge \tilde{Z}\right)_{-}=\left(Z^{*} \wedge \tilde{W}\right)_{-}$we have

$$
\begin{aligned}
& \left.\left\langle[\pi \tilde{Z}, \pi \tilde{W}]_{-}, G_{-}^{A}[\pi \tilde{Z}, \pi \tilde{W}]_{-}\right\rangle-[\tilde{Z}, \tilde{W}]_{-}, G_{-}^{A}[\tilde{Z}, \tilde{W}]_{-}\right\rangle \\
= & \left\{\left\langle\left[\xi_{Z}, \pi \tilde{W}\right]_{-},\left(Z^{*} \wedge \pi \tilde{W}\right)_{-}\right\rangle+\left\langle\left[\xi_{Z}, \tilde{W}\right]_{-},\left(Z^{*} \wedge \tilde{W}\right)_{-}\right\rangle\right. \\
& \left.+\left\langle\left[\pi \tilde{W}, \xi_{Z}\right]_{-},\left(W^{*} \wedge \xi_{Z}\right)_{-}\right\rangle+(W \leftrightarrow Z)\right\} \\
& +\left\langle\left(Z^{*} \wedge \xi_{W}\right)_{-}+\left(W^{*} \wedge \xi_{Z}\right)_{-}, R_{Z W}\right\rangle \\
& +\left\langle R_{Z W}, G_{-}^{A} R_{Z W}\right\rangle+\left\langle R_{Z W}, G_{-}^{A}\left[\xi_{Z}, \xi_{W}\right]_{-}\right\rangle+\left\langle\left[\xi_{Z}, \xi_{W}\right]_{-}, G_{-}^{A}\left[\xi_{Z}, \xi_{W}\right]_{-}\right\rangle
\end{aligned}
$$

To handle the difference arising from the third term in num $(\cdot, \cdot ; A)$ we write

$$
\begin{aligned}
& \left\langle[\pi \tilde{Z}, \pi \tilde{Z}]_{-}, G_{-}^{A}[\pi \tilde{W}, \pi \tilde{W}]_{-}\right\rangle-\left\langle[\tilde{Z}, \tilde{Z}]_{-}, G_{-}^{A}[\tilde{W}, \tilde{W}]_{-}\right\rangle \\
= & \frac{1}{2}\left\langle G_{-}^{A}\left([\pi \tilde{Z}, \pi \tilde{Z}]_{-}-[\tilde{Z}, \tilde{W}]\right),[\pi \tilde{W}, \pi \tilde{W}]_{-}+[\tilde{W}, \tilde{W}]_{-}\right\rangle+(W \leftrightarrow Z) \\
= & \frac{1}{2}\left\langle G_{-}^{A}\left(2\left[\tilde{Z}, \xi_{Z}\right]_{-}+\left[\xi_{Z}, \xi_{Z}\right]_{-}\right), 2[\tilde{W}, \pi \tilde{W}]_{-}+\left[\xi_{W}, \xi_{W}\right]_{-}\right\rangle+(W \leftrightarrow Z) \\
= & \left\langle[\pi \tilde{W}, \pi \tilde{W}]_{-}+[\tilde{W}, \tilde{W}]_{-},\left(Z^{*} \wedge \xi_{Z}\right)_{-}\right\rangle+\left\langle\left[\xi_{Z}, \xi_{Z}\right]_{-},\left(W^{*} \wedge \pi \tilde{W}\right)_{-}\right\rangle \\
& +2\left\langle\left(W^{*} \wedge \pi \tilde{W}\right)_{-}, \mathrm{R}_{2}\left(\tilde{Z}, \xi_{Z}\right)\right\rangle+\left\langle R_{Z Z}, G_{-}^{A}\left[\xi_{W}, \xi_{W}\right]_{-}\right\rangle \\
& +\left\langle R_{W W}, G_{-}^{A} R_{Z Z}\right\rangle+\frac{1}{2}\left\langle\left[\xi_{Z}, \xi_{Z}\right]_{-}, G_{-}^{A}\left[\xi_{W}, \xi_{W}\right]_{-}\right\rangle+(W \leftrightarrow Z)
\end{aligned}
$$

To obtain $\sigma(\pi \tilde{Z}, \pi \tilde{W})-\tilde{\sigma}(\tilde{Z}, \tilde{W})$ we multiply (8.4) by 3 , subtract (8.5), and add (8.6). The terms not involving any Green operator or remainder $\mathrm{R}_{i}$ combine to yield

$$
Q\left(\xi_{Z}, \pi \tilde{W}, Z, \pi \tilde{W}\right)+Q\left(\xi_{Z}, \tilde{W}, Z, \tilde{W}\right)+Q\left(\pi \tilde{W}, \xi_{Z}, W, \xi_{Z}\right)+(W \leftrightarrow Z)
$$


where $Q(\alpha, \eta, Z, \eta)$ denotes the left-hand side of (2.23). By Lemma 2.4,

$$
Q(\alpha, \eta, Z, \eta) \equiv 0
$$

so that only the terms in (8.4-8.6) containing Green operators or remainders contributes to $\tilde{\sigma}-\sigma$. (This cancellation is fortuitous since if we choose $Z=Z_{\mathbf{a}},|\mathbf{a}|=1$ and $W=\lambda^{-1} Z^{\prime}$-in which case the denominator of the sectional curvature formula approaches a nonzero constant-then for example $\left\langle\left\{\xi_{Z}, \tilde{W}\right\}, \iota_{Z} \tilde{W}\right\rangle \sim \lambda^{-1}$ as $\lambda \rightarrow 0$.) To bound the remaining terms we must estimate the remainders $R_{i}$ in several cases. Below, we let

$$
\xi_{(\mathbf{b}, k)}=\left(\pi_{A}-I d\right)\left(\tilde{Z}_{\mathbf{b}}+k \tilde{Z}^{\prime}\right) .
$$

Proposition 8.1. For $i=0,2$ the remainders $R_{i}$ satisfy the following inequalities.

(a)

$$
\begin{aligned}
\left|R_{i}\left(\tilde{Z}_{\mathbf{b}}+k \tilde{Z}^{\prime}, \alpha\right)\right| \leq c \beta^{2}\{(|\mathbf{b}|+|k| r) & \left(|\alpha|+r|\nabla \alpha|+\left|\Delta_{1}^{A} \alpha\right|\right) \\
& \left.+(2-i)|k|\left|d^{A^{*}} \alpha\right|+i|k|\left|d_{-}^{A} \alpha\right|\right\} .
\end{aligned}
$$

(b)

$$
\begin{aligned}
& \begin{aligned}
\mid R_{i}\left(\tilde{Z}_{\mathrm{b}_{1}}+k_{1} \tilde{Z}^{\prime}, \tilde{Z}_{\mathrm{b}_{2}}+\right. & \left.k_{2} \tilde{Z}^{\prime}\right) \mid \\
& \leq c \beta^{2}\left(\left|\mathbf{b}_{1}\right|+\left|k_{1}\right| r\right)\left(\left|\mathbf{b}_{2}\right|+\left|k_{2}\right| r\right)\left(|F|+r\left|\nabla^{A} F\right|\right)
\end{aligned} \\
& \begin{array}{l}
\left|R_{i}\left(\tilde{Z}_{\mathrm{b}_{1}}+k_{1} \tilde{Z}^{\prime}, \xi_{\left(\mathbf{b}_{2}, k_{2}\right)}\right)\right| \leq c \beta^{2}\left(\left|\mathbf{b}_{1}\right|\right. \\
\left.\quad+\left|k_{1}\right| r\right)\left(\left|\xi_{\left(\mathbf{b}_{2}, k_{2}\right)}\right|+r\left|\nabla^{A} \xi_{\left(\mathbf{b}_{2}, k_{2}\right)}\right|+\left(\left|\mathbf{b}_{2}\right|+\left|k_{2}\right| r\right)\left(|F|+r\left|\nabla^{A} F\right|\right)\right)
\end{array}
\end{aligned}
$$

(c)

Proof. (a) Again it suffices to replace $\beta$ by 1 in the proof. From (2.4-2.5) we have

$$
\left|\mathrm{R}_{i}(\tilde{Z}, \alpha)\right| \leq c\left\{(|\Delta Z|+|Z|)|\alpha|+|\nabla Z|\left|\nabla^{A} \alpha\right|+|Z|\left|\Delta_{1}^{A} \alpha\right| \cdot\right\}
$$

If $k=0$, the stated inequality follows immediately from equations (2.4-2.5) and Lemma 5.4, so by linearity it suffices to assume $\mathbf{b}=0, k=1$. First consider the case $i=0$. The only parts of the bound that are not apparent 
are the term proportional to the first derivatives of $\alpha$. But we can use (5.15) to estimate the troublesome term in (2.4), namely

$$
\begin{aligned}
\iota_{\nabla_{a} Z^{\prime}} \nabla_{a} \alpha & =g^{i j} \iota_{\nabla_{\partial / \partial x^{i}} Z^{\prime}} \nabla_{\partial / \partial x^{j}} \alpha \\
& =\left(\delta_{i j}+O\left(r^{2}\right)\right) \iota_{\partial / \partial x^{i}} \nabla_{\partial / \partial x^{j}} \alpha \\
& =-d^{A^{*}} \alpha+O\left(r^{2}|\nabla \alpha|\right),
\end{aligned}
$$

so (8.7) follows. Similarly when $i=2$ we have

$$
\begin{aligned}
p_{-}\left(\nabla_{a} Z^{\prime} \wedge \nabla_{a} \alpha\right) & =p_{-}\left(d x^{i} \wedge \nabla_{\partial / \partial x^{i}} \alpha+O\left(r^{2}|\nabla \alpha|\right)\right. \\
& =d_{-}^{A} \alpha+O\left(r^{2}|\nabla \alpha|\right)
\end{aligned}
$$

and we again obtain (8.7).

(b) Let $Z=Z_{\mathbf{b}_{1}}+k_{1} Z^{\prime}, W=Z_{\mathbf{b}_{2}}+k_{2} Z^{\prime}$, and $\alpha=\tilde{W}=\iota_{W} F$. Using part (a) and Lemma 5.2, we have

$$
\mathrm{R}_{i}(\tilde{Z}, \tilde{W}) \leq c \beta^{2}\left(\left|\mathbf{b}_{1}\right|+\left|k_{1}\right| r\right)\left(|\tilde{W}|+r|\nabla \tilde{W}|+\left|\nabla H^{0}\left(\phi_{\mathrm{b}_{2}}+k_{2} \phi^{\prime}\right) দ F\right|\right) .
$$

From (5.13-5.15) we have

$$
\begin{aligned}
\left|H^{0}\left(\phi_{\mathbf{b}}+k \phi^{\prime}\right)\right| & \leq c r(|\mathbf{b}|+|k| r), \\
\left|\nabla H^{0}\left(\phi_{\mathbf{b}}+k \phi^{\prime}\right)\right| & \leq c(|\mathbf{b}|+|k| r),
\end{aligned}
$$

as well as bounds on the other terms above, and we obtain (8.8).

(c) Let $Z, W$ be as in (b), let $\xi=\xi_{(\mathrm{b}, k)}$, and let $\phi=\phi_{\mathrm{b}_{2}}+k_{2} \phi^{\prime}$. Since $\pi \tilde{W}=\tilde{W}+\xi \in H_{A}$, Lemma 5.2 implies $d^{A^{*}} \xi=0$ and $d_{-}^{A} \xi=-H^{0} \phi \natural F$. Hence, using (2.4) and part (a), $\Delta_{1}^{A} \xi=-2 d_{-}^{A^{*}}\left(H^{0} \phi \curvearrowleft F\right)$, and

$$
\mathrm{R}_{2}(\tilde{Z}, \xi)=p_{-}\left(Z^{*} \wedge d_{-}^{A^{*}}\left(H^{0} \phi \natural F\right)\right)+O\left(\beta^{2}\left(\left|\mathbf{b}_{1}\right|+\left|k_{1}\right| r\right)(|\xi|+r|\nabla \xi|)\right),
$$

with a similar formula for $\mathrm{R}_{0}(\tilde{Z}, \xi)$. Using (8.11) the desired bound follows as in (b).

Applying Proposition 8.1, (8.12), Proposition 5.1, and Corollary 3.4 we obtain the following.

Corollary 8.2. Let $Z=Z_{\mathbf{a}}, W=Z_{\mathbf{b}}+k Z^{\prime}$. Then

$$
\begin{aligned}
\left\|R_{Z Z}\right\|_{2} & \leq c|\mathbf{a}|^{2} . \\
\left\|R_{Z W}\right\|_{2} & \leq c|\mathbf{a}|(|\mathbf{b}|+|k| \lambda) .
\end{aligned}
$$




$$
\begin{aligned}
\left\|R_{W W}\right\|_{2} & \leq c\left(|\mathbf{b}|^{2}+k^{2} \lambda^{2}|\log \lambda|^{1 / 2}\right) . \\
\left\|r R_{Z Z}\right\|_{2} & \leq c|\mathbf{a}|^{2} \lambda . \\
\left\|r R_{Z W}\right\|_{2} & \left.\leq c|\mathbf{a}|\left(|\mathbf{b}| \lambda+|k| \lambda^{2}|\log \lambda|^{1 / 2}\right)\right) . \\
\left\|r R_{W W}\right\|_{2} & \leq c\left(|\mathbf{b}|^{2} \lambda+k^{2} \lambda^{2}\right) .
\end{aligned}
$$

$$
\left\|R_{2}\left(\tilde{Z}, \xi_{Z}\right)-p_{-}\left(Z^{*} \wedge d_{-}^{A^{*}}\left(H^{0} \phi_{\mathbf{a}} \curvearrowleft F\right)\right)\right\|_{2} \leq c|\mathbf{a}|^{2} \lambda^{2}|\log \lambda|^{1 / 2}
$$

Using these estimates, Corollaries 3.4 and 3.6, Proposition 5.1, Lemma 5.4, and (5.1) to bound most of the terms in (8.2) and (8.4-8.6) remaining after the cancellation discussed earlier, we can rewrite the numerator in (8.1) as

$$
\begin{aligned}
\frac{3}{2}\left\langle\iota_{Z} \tilde{W}, \mathrm{R}_{0}(\tilde{Z}, \tilde{W})\right\rangle-\left\langle\left(Z^{*} \wedge \tilde{W}\right)_{-}, \mathrm{R}_{2}(\tilde{Z}, \tilde{W})\right\rangle+\left\langle\left(W^{*} \wedge \tilde{W}\right)_{-}, \mathrm{R}_{2}(\tilde{Z}, \tilde{Z})\right\rangle \\
+2\left\langle\left(W^{*} \wedge \pi \tilde{W}\right)_{-}, \mathrm{R}_{2}\left(\tilde{Z}, \xi_{Z}\right)\right\rangle+2\left\langle\left(Z^{*} \wedge \pi \tilde{Z}\right)_{-}, \mathrm{R}_{2}\left(\tilde{W}, \xi_{W}\right)\right\rangle \\
+ \text { terms involving } G_{0}^{A}+O\left(\lambda|\mathbf{a}|^{2}\left(|\mathbf{b}|^{2}+k^{2} \lambda^{2}\right)\right) .
\end{aligned}
$$

It is easy to check using the tools above that the first two terms in (8.14) are $O\left(|\mathbf{a}|^{2}\left(|\mathbf{b}|^{2}+k^{2} \lambda^{2}\right)\right)$. So are the third and fourth terms, as we see by noting that $\left.\int \beta\left(|\mathrm{b}|^{2}+k^{2} r^{2}\right)|F||\mathrm{R}|\right) \leq c\left(|\mathrm{~b}|^{2}\|\mathrm{R}\|_{2}+k^{2}\|\beta r F\|_{2}\|r \mathrm{R}\|_{2}\right)$. Finally, we get the same bound for the fifth term, using (8.9) directly rather than Corollary 8.2 to do the estimate.

It remains to estimate the four terms in (8.2) and (8.4) involving $G_{0}^{A}$. This requires some delicacy because the eigenvalues of $\Delta_{0}^{A}$ are not bounded away from zero; in fact it can be shown that the first three eigenvalues are bounded above and below by positive multiples of $\lambda^{2}$. This fact is widely known, but a proof does not seem to exist in the literature, so we give a proof of the lower bound - which for us is the important one - in the appendix. It is also known that all but three $(=\operatorname{dim}(\operatorname{Ad} P))$ eigenvalues are uniformly bounded away from zero (see [T1, Lemma 6.10]). Thus we have the following characterization of the eigenvalues of $\Delta_{0}^{A}$ in $\mathcal{M}_{\lambda_{0}}$.

Proposition 8.3. Let $\left\{\mu_{i}(A)\right\}_{1}^{\infty}$ be the eigenvalues of $\Delta_{0}^{A}$, indexed in nondecreasing order. Then there are positive constants $c_{1}, c_{2}$ such that for all 
$A \in \mathcal{M}_{\lambda_{0}}$,

$$
c_{1} \lambda^{2} \leq \mu_{i} \leq c_{2}, \quad i \leq 3
$$

and

$$
\mu_{i} \geq c_{2}, \quad i>3 .
$$

Let $V_{A}$ denote the span of the first three eigensections of $\Delta_{0}^{A}$, and $V_{A}^{\perp}$ its $L^{2}$-orthogonal complement. Writing $R^{V}$ and $R^{\perp}$ for the corresponding components of any $R \in \Omega^{0}(\operatorname{Ad} P$ ) (suppressing the $A$-dependence) we have

$$
\left\langle R_{1}, G_{0}^{A}\left(R_{2}\right)\right\rangle=\left\langle R_{1}^{V}, G_{0}^{A}\left(R_{2}^{V}\right)\right\rangle+\left\langle R_{1}^{\perp}, G_{0}^{A}\left(R_{2}^{\perp}\right)\right\rangle .
$$

Since by Proposition $8.3 G_{0}^{A} \circ \pi_{V}^{\perp}$ is uniformly bounded below (where $\pi_{V}^{\perp}$ is the orthogonal projection to $V_{A}^{\perp}$ ), an argument similar to the proof of (3.20) implies that

$$
\left|\left\langle R_{1}^{\perp}, G_{0}^{A} R_{2}^{\perp}\right\rangle\right| \leq c\left\|r R_{1}^{\perp}\right\|_{2}\left\|r R_{2}^{\perp}\right\|_{2} \leq c\left(\left\|r R_{1}\right\|_{2}+\left\|R_{1}^{V}\right\|_{2}\right)\left(\left\|r R_{2}\right\|_{2}+\left\|R_{2}^{V}\right\|_{2}\right) .
$$

To estimate the other term in (8.17) we also will need a bound on $\left\|R^{V}\right\|_{2}$. For the $R$ 's under consideration, one expects this quantity to be small, since the sections comprising $V_{A}$ are, loosely speaking, supported mostly away from $p$-they can be closely approximated by sections that are almost covariantly constant outside a small ball around $p$ and are zero on a smaller ball- while our $R$ 's are supported mostly near $p$. The next set of lemmas quantifies these ideas.

Lemma 8.4. Let $\alpha \in \Omega^{1}(\operatorname{Ad} P)$ satisfy $d^{A^{*}} \alpha=0$ and let $\psi \in \Omega^{0}(\operatorname{Ad} P)$. Then

$$
\begin{aligned}
\left|\left\langle R_{0}\left(\tilde{Z}_{\mathrm{b}}+k \tilde{Z}^{\prime}, \alpha\right), \psi\right\rangle\right| \leq c\left(\|\beta(|\mathbf{b}|+|k| r) \alpha\|_{1}\|\psi\|_{\infty}\right. \\
\left.+\left(\left\|\beta(|\mathbf{b}|+|k| r) d_{-}^{A} \alpha\right\|_{2}+\left\|\beta\left(|\mathbf{b}| r+|k| r^{2}\right) \alpha\right\|_{2}\right)\left\|d_{A} \psi\right\|_{2}\right) .
\end{aligned}
$$

Proof. Let $Z=Z_{\mathbf{b}}+k Z^{\prime}$ and $R=\mathrm{R}_{0}(\tilde{Z}, \alpha)$. From (2.4) we have

$$
\begin{aligned}
\left|\left\langle\mathrm{R}_{0}\left(\tilde{Z}_{\mathrm{b}}+k \tilde{Z}^{\prime}, \alpha\right), \psi\right\rangle\right| \leq c(\|(|\Delta Z| & +|Z|) \alpha\left\|_{1}\right\| \psi \|_{\infty} \\
& \left.+\left|\left\langle\iota_{Z} d_{-}^{A^{*}} d_{-}^{A} \alpha, \psi\right\rangle\right|+\left|\left\langle\iota_{\nabla_{a} Z} \nabla_{a}^{A} \alpha, \psi\right\rangle\right|\right) .
\end{aligned}
$$


The first term on the right is bounded by the first term in (8.19). We integrate the second term in (8.20) by parts. Since $Z$ is a gradient vector field, $d Z^{*}=0$; thus

$$
\begin{aligned}
& \left|\left\langle\iota_{Z} d_{-}^{A^{*}} d_{-}^{A} \alpha, \psi\right\rangle\right|=\left|\left\langle d_{-}^{A^{*}} d_{-}^{A} \alpha, \psi \otimes Z^{*}\right\rangle\right| \\
& \quad=\left|\left\langle d_{-}^{A} \alpha, d_{A} \psi \wedge Z^{*}\right\rangle\right| \leq\left\||Z| d_{-}^{A} \alpha\right\|_{2}\left\|d^{A} \psi\right\|_{2},
\end{aligned}
$$

which is bounded by the second term in (8.19). For the third term in (8.20), we use the derivation of (8.10) and the assumption $d^{A^{*}} \alpha=0$ to write $\iota_{\nabla_{a}} Z \nabla_{a}^{A} \alpha=$ $\iota_{U_{a}} \nabla_{a}^{A} \alpha$, where $|U| \leq c \beta\left(|\mathbf{b}| r+|k| r^{2}\right)$ and $|\nabla U| \leq c \beta(|\mathbf{b}|+|k| r)$. We then have

$$
\begin{aligned}
\left|\left\langle\iota_{U_{a}} \nabla_{a} \alpha, \psi\right\rangle\right|=\left|\left\langle\nabla \alpha, \psi \otimes U^{*}\right\rangle\right| & =\left|\left\langle\alpha, \nabla^{*}(\psi \otimes U)\right\rangle\right| \\
& \leq\left\|U\left|\alpha\left\|_{2}\right\| d^{A} \psi\left\|_{2}+\right\|\right| \nabla U \mid \alpha\right\|_{1}\|\psi\|_{\infty}
\end{aligned}
$$

and (8.19) follows.

Our intention of course is to apply this lemma to $\psi \in V_{A}$. For such $\psi$ we have the following.

Lemma 8.5. There is a constant $c$ such that for $A \in \mathcal{M}_{\lambda_{0}}$ and $\psi \in V_{A}$,

$$
\|\psi\|_{\infty} \leq c\|\psi\|_{2} .
$$

Proof. For $1 \leq i \leq 3$ let $\mu_{i}$ be as in Proposition 8.3 and let $\psi_{i}$ be corresponding eigensections. Since $\operatorname{dim}\left(V_{A}\right)=3$ independent of $A$ it suffices to prove the result for each $\psi_{i}$.

We use a simple fact from elliptic theory: given a constant $\mu$, there exists $c=$ $c(\mu)$ such that if $f \in L_{1}^{2}(M)$ is nonnegative and satisfies $\Delta f \leq \mu f$ pointwise, then

$$
\|f\|_{\infty} \leq c\|f\|_{2}
$$

(This follows immediately from Theorem 9.20 of [GT].) But a relative of Kato's inequality (3.9) gives

$$
\Delta\left|\psi_{i}\right| \leq\left|\psi_{i}\right|^{-1}\left(\psi_{i}, \Delta_{A} \psi_{i}\right)=\mu_{i}\left|\psi_{i}\right| \leq c_{2}\left|\psi_{i}\right|
$$

where $c_{2}$ is as in (8.15), so (8.23) implies the result. 
Lemma 8.6. Let $W=Z_{\mathbf{b}_{2}}+k_{2} Z^{\prime}$, let $\alpha$ be either $\tilde{W}$ or $\xi_{W}$, let $b_{2}=\left|\mathbf{b}_{2}\right|$, and assume $k_{1} k_{2}=0$. Then

$$
\left\|\beta\left(b_{1}+\left|k_{1}\right| r\right) \alpha\right\|_{1} \leq c\left(b_{1} b_{2} \lambda^{2}|\log \lambda|+\left(b_{1}\left|k_{2}\right|+b_{2}\left|k_{1}\right|\right) \lambda^{2}\right)
$$

and

$\left\|\beta\left(b_{1}+\left|k_{1}\right| r\right) d_{-}^{A} \alpha\right\|_{2}+\left\|\beta\left(b_{1} r+\left|k_{1}\right| r^{2}\right) \alpha\right\|_{2} \leq c\left(b_{1} b_{2} \lambda+\left(b_{1}\left|k_{2}\right|+b_{2}\left|k_{1}\right|\right) \lambda^{2}|\log \lambda|^{1 / 2}\right)$.

Proof. We leave most of this to the reader, since the details are similar to those of our previous estimates. The only estimate we have not encountered before is (8.24) in the case $\alpha=\xi_{W}$. For this we use compactness of $M$ to bound the $L^{1}$ norm by the $L^{2}$ norm, and use (5.2) and (5.4) to note that $\left\|r \xi_{W}\right\|_{2} \leq c\left(b_{2}+\left|k_{2}\right|\right) \lambda^{2}$.

Remark 8.1. Were it not for (5.4), we would have $b_{2}\left|k_{1}\right| \lambda^{2}|\log \lambda|^{1 / 2}$ on the right-hand side of (8.24) when $\alpha=\xi_{W}$. Using this to estimate the term

$$
\left\langle\mathrm{R}_{0}(\tilde{Z}, \pi \tilde{W})-\mathrm{R}_{0}(\tilde{W}, \pi \tilde{Z}), G_{0}^{A}\left(\mathrm{R}_{0}\left(\tilde{Z}, \xi_{W}\right)-\mathrm{R}_{0}\left(\tilde{W}, \xi_{Z}\right)\right)\right\rangle
$$

in (8.4) would then give only a logarithmically divergent upper bound. This is the sole reason we need (5.4), and thus Theorem 6.1 .

Corollary 8.7. Let $\psi \in V_{A}$ satisfy $\Delta_{0}^{A} \psi=\mu \psi$ and $\|\psi\|_{2}=1$. There are constants $c$, independent of $\mu$, such that

$$
\left|\left\langle R_{Z W}, \psi\right\rangle\right| \leq c|\mathbf{a}|\left\{|\mathbf{b}| \lambda^{2}|\log \lambda|+|k| \lambda^{2}+\mu^{1 / 2}\left(|\mathbf{b}| \lambda+|k| \lambda^{2}|\log \lambda|^{1 / 2}\right)\right\} .
$$

$$
\left\|R_{Z W}^{V}\right\|_{2} \leq c|\mathbf{a}|\left(|\mathbf{b}| \lambda+|k| \lambda^{2}|\log \lambda|^{1 / 2}\right)
$$

$$
\left|\left\langle\left\{\xi_{Z}, \xi_{W}\right\}, \psi\right\rangle\right|+\left\|\left\{\xi_{Z}, \xi_{W}\right\}^{V}\right\|_{2} \leq c|\mathbf{a}| \lambda^{3}|\log \lambda|^{1 / 2}\left(|\mathbf{b}| \lambda|\log \lambda|^{1 / 2}+|k| \lambda\right) .
$$

Proof. Let $\alpha$ be as in Lemma 8.6. Combining Lemmas 8.4-8.6 we have

$$
\begin{aligned}
\left|\left\langle\mathrm{R}_{0}\left(\tilde{Z}_{\mathbf{b}_{1}}+k \tilde{Z}^{\prime}, \alpha\right), \psi\right\rangle\right| \leq & c\left\{\left|\mathbf{b}_{1}\right|\left|\mathbf{b}_{2}\right| \lambda^{2}|\log \lambda|+\left(\left|\mathbf{b}_{1}\right|\left|k_{2}\right|+\left|\mathbf{b}_{2}\right|\left|k_{1}\right|\right) \lambda^{2}\right) \\
& \left.+\mu^{1 / 2}\left(\left|\mathbf{b}_{1}\right|\left|\mathbf{b}_{2}\right| \lambda+\left(\left|\mathbf{b}_{1}\right|\left|k_{2}\right|+\left|\mathbf{b}_{2}\right|\left|k_{1}\right|\right) \lambda^{2}|\log \lambda|^{1 / 2}\right)\right\}
\end{aligned}
$$


from which (8.25) follows. To obtain (8.26), write $\left\|R^{V}\right\|_{2}^{2}=\sum_{i=1}^{3}\left\langle R, \psi_{i}\right\rangle^{2}$, where $\left\{\psi_{i}\right\}$ is an orthonormal eigenbasis of $V_{A}$, and use (8.25) and Proposition 8.3. Similarly, we obtain (8.27) with the aid of (5.2).

While (8.26) is good enough for our purposes, in actuality the constant $c_{2}$ in Proposition 8.3 is $O\left(\lambda^{2}\right)$, so (8.26) could be improved.

We apply Corollaries 8.7 and 8.2 to estimate the terms involving $G_{0}^{A}$ in (8.2) and (8.4). Letting $Z, W$, and $R_{Z W}$ be as in Corollary 8.2 (with $R=\mathrm{R}_{0}$ ) we have

$$
\left\|r R_{Z W}\right\|_{2}+\left\|R_{Z W}^{V}\right\|_{2} \leq c|\mathbf{a}|\left(|\mathbf{b}| \lambda+|k| \lambda^{2}|\log \lambda|^{1 / 2}\right) .
$$

Furthermore we have

$$
\begin{aligned}
& \left\|r\left\{\xi_{Z}, \xi_{W}\right\}\right\|_{2}+\left\|\left\{\xi_{Z}, \xi_{W}\right\}^{V}\right\|_{2} \\
& \quad \leq c\left\|\xi_{Z}\right\|_{4}\left\|r \xi_{W}\right\|_{4}+\left\|\left\{\xi_{Z}, \xi_{W}\right\}^{V}\right\|_{2} \leq c|\mathbf{a}| \lambda^{2}\left(|\mathbf{b}| \lambda|\log \lambda|^{1 / 2}+|k| \lambda\right) .
\end{aligned}
$$

Now each term involving $G_{0}^{A}$ is one of the types

$$
\left\langle R_{Z W}, G_{0}^{A}\left(R_{Z W}\right)\right\rangle,\left\langle R_{Z W}, G_{0}^{A}\left\{\xi_{Z}, \xi_{W}\right\}\right\rangle \text {, or }\left\langle\left\{\xi_{Z}, \xi_{W}\right\}, G_{0}^{A}\left\{\xi_{Z}, \xi_{W}\right\}\right\rangle .
$$

Let $\left\{\psi_{i}\right\}$ be an orthonormal eigenbasis of $V_{A}$ with eigenvalues $\mu_{i}$. Then using (8.15) and the estimates above we have

$$
\begin{aligned}
\left|\left\langle R_{Z W}^{V}, G_{0}^{A}\left(R_{Z W}^{V}\right)\right\rangle\right| & =\left|\sum_{i=1}^{3} \mu_{i}^{-1}\left\langle R_{Z W}, \psi_{i}\right\rangle\left\langle R_{Z W}, \psi_{i}\right\rangle\right| \\
& \leq c|\mathbf{a}|^{2}\left\{\lambda^{-2}\left(|\mathbf{b}|^{2} \lambda^{4}|\log \lambda|^{2}+k^{2} \lambda^{4}\right)+|\mathbf{b}|^{2} \lambda^{2}+k^{2} \lambda^{4}|\log \lambda|\right\} \\
& \leq c|\mathbf{a}|^{2}\left(|\mathbf{b}|^{2} \lambda^{2}|\log \lambda|^{2}+k^{2} \lambda^{2}\right) .
\end{aligned}
$$

We can estimate $\left|\left\langle R_{Z W}^{V}, G_{0}^{A}\left\{\xi_{Z}, \xi_{W}\right\}^{V}\right\rangle\right|$ and $\left|\left\langle\left\{\xi_{Z}, \xi_{W}\right\}^{V}, G_{0}^{A}\left\{\xi_{Z}, \xi_{W}\right\}^{V}\right\rangle\right|$ in a similar fashion. Using (8.17),(8.18), and (8.28- 8.29), we obtain the following bounds, with room to spare.

$$
\left\langle R_{Z W}, G_{0}^{A}\left(R_{Z W}\right)\right\rangle=\left\langle R_{Z W}^{V}, G_{0}^{A}\left(R_{Z W}^{V}\right)\right\rangle+O\left(\lambda|\mathbf{a}|^{2}\left(|\mathbf{b}|^{2}+k^{2} \lambda^{2}\right)\right) .
$$

$$
\left\langle R_{Z W}^{V}, G_{0}^{A}\left(R_{Z W}^{V}\right)\right\rangle=O\left(|\mathbf{a}|^{2}\left(|\mathbf{b}|^{2} \lambda+k^{2} \lambda^{2}\right)\right) .
$$

$$
\left|\left\langle R_{Z W}, G_{0}^{A}\left\{\xi_{Z}, \xi_{W}\right\}\right\rangle\right|+\left|\left\langle\left\{\xi_{Z}, \xi_{W}\right\}, G_{0}^{A}\left\{\xi_{Z}, \xi_{W}\right\}\right\rangle\right|=O\left(\lambda|\mathbf{a}|^{2}\left(|\mathbf{b}|^{2} \lambda+k^{2} \lambda^{2}\right)\right) .
$$




\section{Boundedness and Partial Continuity of the Curvature}

With the estimates behind us, we can finally prove our main theorems.

Recall that any two-plane in $H_{A}$ has a basis of the form $\pi_{A} \tilde{Z}^{A}, \pi_{A} \tilde{W}^{A}$, where $\tilde{Z}^{A}, \tilde{W}^{A}$ are as in (2.19-2.20). Then the estimates of the previous section show that

$$
\sigma_{A}\left(\pi \tilde{Z}^{A}, \pi \tilde{W}^{A}\right)=\frac{\operatorname{Rem}^{\prime}(Z, W ; A)}{\operatorname{denom}\left(\pi_{A} \tilde{Z}^{A}, \pi_{A} \tilde{W}^{A}\right)}
$$

where $\operatorname{Rem}^{\prime}(Z, W ; A)$, which is written out explicitly in (9.1) below, is

$$
O\left(|\mathbf{a}|^{2}\left(|\mathbf{b}|^{2}+k^{2} \lambda^{2}\right)\right)=O(1)
$$

By (5.2) of this paper and Proposition 3.6 of [GP2]],

$$
\lim _{\lambda \rightarrow 0} \operatorname{denom}\left(\pi_{A} \tilde{Z}^{A}, \pi_{A} \tilde{W}^{A}\right)=\lim _{\lambda \rightarrow 0} \operatorname{denom}\left(\tilde{Z}^{A}, \tilde{W}^{A}\right)=(2 \pi)^{4} .
$$

Hence the curvature is bounded, completing the proof of Theorem 1.1.

To prove Theorem 1.2 we examine $\operatorname{Rem}^{\prime}(Z, W ; A)$ in the case $Z=Z_{\mathbf{a}}, W=$ $W_{\mathbf{b}}$, with $|\mathbf{a}|=|\mathbf{b}|=1$ and $\mathbf{a} \perp \mathbf{b}$. From (8.14) we have

$$
\begin{aligned}
\operatorname{Rem}^{\prime}(Z, W ; A)= & \frac{3}{2}\left\langle\iota_{Z} \tilde{W}, \mathrm{R}_{0}(\tilde{Z}, \tilde{W})\right\rangle-\left\langle\left(Z^{*} \wedge \tilde{W}\right)_{-}, \mathrm{R}_{2}(\tilde{Z}, \tilde{W})\right\rangle \\
& +\left\langle\left(W^{*} \wedge \tilde{W}\right)_{-}, \mathrm{R}_{2}(\tilde{Z}, \tilde{Z})\right\rangle+2\left\langle\left(W^{*} \wedge \pi \tilde{W}\right)_{-}, \mathrm{R}_{2}\left(\tilde{Z}, \xi_{Z}\right)\right\rangle \\
& +2\left\langle\left(Z^{*} \wedge \pi \tilde{Z}\right)_{-}, \mathrm{R}_{2}\left(\tilde{W}, \xi_{W}\right)\right\rangle+3\left\langle\mathrm{R}_{0}(\tilde{Z}, \tilde{W}), G_{0}^{A}\left(\mathrm{R}_{0}(\tilde{Z}, \tilde{W})\right)\right\rangle \\
& +\left\langle\mathrm{R}_{0}(\tilde{Z}, \pi \tilde{W})-\mathrm{R}_{0}(\tilde{W}, \pi \tilde{Z}), G_{0}^{A}\left(\mathrm{R}_{0}\left(\tilde{Z}, \xi_{W}\right)-\mathrm{R}_{0}\left(\tilde{W}, \xi_{Z}\right)\right)\right\rangle \\
& +O\left(\lambda^{1-\delta}\right)
\end{aligned}
$$

after suppressing some $A$ 's. (We have $O\left(\lambda^{1-\delta}\right)$ here rather than $O(\lambda)$ because are not assuming the $\Lambda_{-}^{2}$ curvature condition here. Because of this our estimates are weaker than before; see the remark following Corollary 3.4. Here and below, $\delta$ does not necessarily mean the $\delta$ in (3.2), but is proportional to it.) Setting $k=0$ in $(8.30-8.32)$ we see that the inner products above 
involving $G_{0}^{A}$ are $O(\lambda)$. Using (5.2) and (8.13) we therefore have

$$
\begin{aligned}
\operatorname{Rem}^{\prime}(Z, W ; A)= & \frac{3}{2}\left\langle\iota_{Z} \tilde{W}, \mathrm{R}_{0}(\tilde{Z}, \tilde{W})\right\rangle-\left\langle\left(Z^{*} \wedge \tilde{W}\right)_{-}, \mathrm{R}_{2}(\tilde{Z}, \tilde{W})\right\rangle \\
& +\left\langle\left(W^{*} \wedge \tilde{W}\right)_{-}, \mathrm{R}_{2}(\tilde{Z}, \tilde{Z})\right\rangle \\
& +2\left\langle\left(W^{*} \wedge \tilde{W}\right)_{-}, Z^{*} \wedge d_{-}^{A^{*}}\left(H^{0} \phi_{\mathbf{a}} \mapsto F\right)\right\rangle \\
& +2\left\langle\left(Z^{*} \wedge \tilde{Z}\right)_{-}, W^{*} \wedge d_{-}^{A^{*}}\left(H^{0} \phi_{\mathbf{b}} \curvearrowleft F\right)\right\rangle+O\left(\lambda^{1-\delta}\right) .
\end{aligned}
$$

Thus as $\lambda \rightarrow 0, \sigma(\pi \tilde{Z}, \pi \tilde{W})$ reduces to a purely local, and hence computable, expression. We will save the detailed computation for [G2]; the qualitative nature of the calculation will suffice here.

If we use (2.4) and (2.5) to write out (9.2) in gory detail as an integral over $M$ (modulo $O\left(\lambda^{1-\delta}\right)$ ), the terms in the integrand can be classified according to how many derivatives of $F$ are involved ( 0 or 1 ) and the way in which the derivatives (if any, up to a total of 2) of the vector fields $Z, W$ and the related functions $\phi_{\mathbf{a}}, \phi_{\mathbf{b}}$ are distributed. Each term is then of one of the following six types, where the meaning of the notation should be obvious.

- type 1: $F^{2} \cdot Z^{2} \cdot W^{2}$

- type 2: $F^{2} \cdot Z \cdot \Delta Z \cdot W^{2}$ or $F^{2} \cdot W \cdot \Delta W \cdot Z^{2}$

- type 3: $F^{2} \cdot Z \cdot \nabla Z \cdot W \cdot \nabla W$ or $F^{2} \cdot Z^{2} \cdot(\nabla W)^{2}$ or $F^{2} \cdot W^{2} \cdot(\nabla Z)^{2}$

- type 4: $F^{2} \cdot Z \cdot \nabla H^{0} \phi_{\mathbf{a}} \cdot W^{2}$ or $F^{2} \cdot W \cdot \nabla H^{0} \phi_{\mathbf{b}} \cdot Z^{2}$

- type 5: $F \cdot \nabla F \cdot Z \cdot \nabla Z \cdot W^{2}$ or $F \cdot \nabla F \cdot W \cdot \nabla W \cdot Z^{2}$

- type 6: $F \cdot \nabla F \cdot Z \cdot H^{0} \phi_{\mathbf{a}} \cdot W^{2}$ or $F \cdot \nabla F \cdot W \cdot H^{0} \phi_{\mathbf{b}} \cdot Z^{2}$.

The type-1 terms occur with coefficients proportional to the endomorphisms $\mathcal{R}=$ Ric, $\mathcal{R}_{\text {_ }}$ that arose from Weitzenböck formulas in our derivations of (2.4) and (2.5). In terms of normal coordinates centered at $p$ we can write $\mathcal{R}=\mathcal{R}(p)+O(r)$. The $O(r)$ term here gives an integrand which is $O\left(r|F|^{2}\right)$, hence an integral which is $O(\lambda)$ which we can ignore. The $O\left(|F|^{2}\right)$ piece is a linear combination of terms of the form $\left(F_{i j}, F_{k l}\right)$ with coefficients that are proportional to $\mathcal{R}(p)$, quadratic in the components of each of $\mathbf{a}$ and $\mathbf{b}$. But as $\lambda \rightarrow 0, A$ approaches a rescaled standard instanton ([D1, Theorem 16.(i)]) and $\left(F_{i j}, F_{k l}\right)$ approaches a $\delta$-function centered at $p$, times a universal constant, times $\delta_{i k} \delta_{j l}-\delta_{i l} \delta_{j k}+\epsilon_{i j k l}$ ( cf. [GP2, Lemma 3.5]). Since $\mathcal{R}$ is constructed naturally from Riem $_{M}$, the Riemann tensor of $(M, g)$, the limit of the integral 
is $\left.L_{1}\left(\operatorname{Riem}_{M}\right)\right|_{p}(\mathbf{a}, \mathbf{b}, \mathbf{b}, \mathbf{a})$ for some geometrically natural endomorphism $L_{1}$.

Similar considerations apply to the other terms. For the terms of type 2 and 4, $\operatorname{Riem}_{M}$ enters from (5.14) rather than from a Weitzenböck formula; for types 5 and 6 it enters from (6.2). In types 2 and 4, if we replace the components of $\operatorname{Riem}_{M}$ in normal coordinates by their values at $p$, the error in the integrand is $O\left(r|F|^{2}\right)$; the same replacement in types 5 and 6 gives an error that is $O\left(r^{2}|F||\nabla F|\right)$. After integration, both of these errors are $O\left(\lambda^{1-\delta}\right)$ and hence ignorable in the limit. The terms we are not throwing away are proportional to $\operatorname{Riem}_{M}(p)$ and to an expression of one of the forms $\left(F_{i j}, F_{k l}\right), x^{m}\left(F_{i j}, \nabla_{m} F_{k l}\right)$, both of which approach delta-functions times universal tensors as above. Finally, the type-3 terms are $O\left(r^{2}|F|^{2}\right)$, and hence integrate to $O\left(\lambda^{2-\delta}\right)$, so we can throw these terms away as well.

Thus $\lim _{\lambda \rightarrow 0} \operatorname{Riem}_{\mathcal{M}}\left(\pi \tilde{Z}_{\mathbf{a}}, \pi \tilde{Z}_{\mathbf{b}}, \pi \tilde{Z}_{\mathbf{b}}, \pi \tilde{Z}_{\mathbf{a}}\right)=L\left(\operatorname{Riem}_{M}\right)(\mathbf{a}, \mathbf{b}, \mathbf{b}, \mathbf{a})$ for some universal endomorphism $L$. By polarization we obtain

$$
\lim _{\lambda \rightarrow 0} \operatorname{Riem}_{\mathcal{M}}\left(\pi \tilde{Z}_{\mathbf{a}}, \pi \tilde{Z}_{\mathbf{b}}, \pi \tilde{Z}_{\mathbf{c}}, \pi \tilde{Z}_{\mathbf{d}}\right)=L\left(\operatorname{Riem}_{M}\right)(\mathbf{a}, \mathbf{b}, \mathbf{c}, \mathbf{d}) .
$$

Now the subspace of $H_{A}$ spanned by the $\pi \tilde{Z}_{\mathbf{a}}$ 's is not precisely the kernel of $d \lambda$-i.e. the tangent space to the leaf $M_{\lambda}$ - but it is close. In fact, by Proposition 5.2 of [GP2] the unit normals to $M_{\lambda}$ and $\operatorname{span}\left\{\pi \tilde{Z}_{\mathbf{a}}\right\}$ differ by $O\left(\lambda^{1-\delta}\right)$ for any $\delta$. Now under the $\Lambda_{-}^{2}$ curvature condition (1.1) implies that $\operatorname{Riem}_{\mathcal{M}}$ is bounded; without this condition the estimates in this paper still show that $\operatorname{Riem}_{\mathcal{M}}=O\left(\lambda^{-\delta}\right)$. Thus the limiting curvature is the same whether we restrict it to the tangent space to $M_{\lambda}$ or to $\operatorname{span}\left\{\pi \tilde{Z}_{\mathbf{a}}\right\}$ We can even eliminate the $\delta$ using the estimates in this paper, but all that matters is that the difference tends to zero; since by Theorem 0.5 , the limiting curvature is the same whichever of these subspaces we restrict it to. This completes the proof of Theorem (1.2).

\section{Appendix A. Tables Pertaining to $\S 7$}

Table1: Several 'projections' $\pi$ above are actually only scalar multiples of projections, as the author found convenient; this does not affect the ranks of any matrices computed in the text. The indices of $X$ and $\pi(X)$ in the table are ordered alphabetically. Our symmetrization convention is that " $Y+\left(i_{1} \ldots i_{k}\right)$ " 
means the expression obtained by adding to $Y$ the minimal number of terms, each of which differs from $Y$ only by a permutation of the indices $\left\{i_{1}, \ldots i_{k}\right\}$ of $Y$, so that the result is symmetric in these indices. In doing so we take into account symmetries of the space in column 2; e.g. for $X \in \mathrm{Sym}_{0}^{2} \otimes \mathbb{R}^{4}$, $X_{a b i}+(a b i)$ is a sum of three terms, not six. Indices surrounded by parentheses are to be symmetrized over the same way; e.g. $\delta_{(i j} \delta_{k l)}=\delta_{i j} \delta_{k l}+(i j k l)$ is a sum of three terms.

\section{FORMULAS FOR PROJECTIONS}

\begin{tabular}{|c|c|c|c|}
\hline projection & domain & image & components of $\pi(T)$ \\
\hline$\pi_{(3,3)}$ & $\mathbb{R}^{4} \otimes \mathbb{R}^{4}$ & $\mathrm{Sym}_{0}^{2}$ & $X_{i j}+X_{j i}-\frac{1}{2} X_{a a} \delta_{i j}$ \\
\hline$\pi_{(4,4)}^{\prime}$ & $\operatorname{Sym}_{0}^{2} \otimes \mathbb{R}^{4}$ & $\mathrm{Sym}_{0}^{3}$ & $X_{(a b i)}-\frac{1}{3}\left[X_{i k k} \delta_{a b}+(a b i)\right]$ \\
\hline$\pi_{(4,4)}$ & $\mathbb{R}^{4} \otimes \mathbb{R}^{4} \otimes \mathbb{R}^{4}$ & $\mathrm{Sym}_{0}^{3}$ & $X_{(i j k)}-\frac{1}{3}\left[\delta_{i j}\left(X_{a a k}+X_{a k a}+X_{k a a}\right)+(i j k)\right]$ \\
\hline$\pi_{(5,5)}$ & $\mathrm{Sym}_{0}^{2} \otimes \mathrm{Sym}_{0}^{2}$ & $\mathrm{Sym}_{0}^{4}$ & $\begin{array}{l}\frac{1}{3} X_{(i j k l)}-\frac{1}{12}\left[\delta_{i j}\left(X_{a k a l}+(k l)\right)+(i j k l)\right]+ \\
\frac{1}{36} X_{a b a b} \delta_{(i j} \delta_{k l)}\end{array}$ \\
\hline$\pi_{5335}$ & $\mathrm{Sym}_{0}^{2} \otimes \mathrm{Sym}_{0}^{2}$ & $\begin{array}{l}(5,3) \oplus(3,5)- \\
\quad \text { subspace }\end{array}$ & $\begin{array}{l}X_{i j k l}-X_{k l i j} \\
\quad-\frac{1}{6}\left\{\left[\delta_{i k}\left(X_{a j a l}-(j l)\right)+(i j)\right]+(k l)\right\}\end{array}$ \\
\hline$\pi_{5115}$ & $\mathrm{Sym}_{0}^{2} \otimes \mathrm{Sym}_{0}^{2}$ & $\begin{array}{l}(5,1) \oplus(1,5)- \\
\text { subspace }\end{array}$ & $\begin{array}{l}\frac{1}{3}\left(2 X_{i j k l}+2 X_{k l i j}-X_{i k j l}\right. \\
\left.\quad-X_{j l i k}-X_{i l j k}-X_{j k i l}\right) \\
+\frac{1}{3}\left[\delta_{i j}\left(X_{a k a l}+(k l)\right)+\delta_{k l}\left(X_{a i a j}+(i j)\right)\right] \\
-\frac{1}{6}\left\{\left[\delta_{i k}\left(X_{a j a l}+(j l)\right)+(i j)\right]+(k l)\right\} \\
+X_{a b a b}\left(\frac{1}{9} \delta_{k(i} \delta_{j) l}-\frac{2}{9} \delta_{i j} \delta_{k l}\right)\end{array}$ \\
\hline$\pi_{(6,6)}$ & $\mathrm{Sym}_{0}^{2} \otimes \mathrm{Sym}_{0}^{3}$ & $\operatorname{Sym}_{0}^{5}$ & $\begin{array}{l}\frac{1}{10} X_{(i j k l m)}-\frac{1}{50}\left[\delta_{i j}\left(X_{a k a l m}+(k l m)\right)+\right. \\
\left.(i j k l m)]+\frac{1}{200}\left[X_{a b a b i} \delta_{(j k} \delta_{l m}\right)+(i j k l m n)\right]\end{array}$ \\
\hline$\pi_{6446}$ & $\mathrm{Sym}_{0}^{2} \otimes \mathrm{Sym}_{0}^{3}$ & $\begin{array}{l}(6,4) \oplus(4,6)- \\
\text { subspace }\end{array}$ & $\begin{array}{l}\frac{1}{15}\left\{6 X_{i j k l m}+\left[\left(X_{i k j l m}+(k l m)\right)+(i j)\right]\right. \\
\left.-4\left[X_{k l i j m}+(k l m)\right]\right\}-\frac{2}{75} \delta_{i j}\left[X_{a k a l m}+\right. \\
(k l m)]-\frac{3}{50}\left[\left(\delta_{i k} X_{a j a l m}+(k l m)\right)+(i j)\right] \\
+\frac{7}{300}\left[\left(\delta_{i k} X_{a l a m j}+(k l m)\right)+(i j)\right] \\
+\frac{11}{150}\left[\delta_{k l} X_{a m a i j}+(k l m)\right] \\
-\frac{1}{100}\left[\left(\delta_{k l} X_{a i a j m}+(k l m)\right]+(i j)\right] \\
-\frac{1}{100}\left[X_{a b a b i} \delta_{j(k} \delta_{l m)}+(i j)\right] \\
+\frac{1}{150} \delta_{i j} X_{a b a b(k} \delta_{l m)} \\
+\frac{1}{150}\left[X_{a b a b k} \delta_{l(i} \delta_{j) m}+(k l m)\right]\end{array}$ \\
\hline$\pi_{6226}$ & $\mathrm{Sym}_{0}^{2} \otimes \mathrm{Sym}_{0}^{3}$ & $\begin{array}{l}(6,2) \oplus(2,6)- \\
\text { subspace }\end{array}$ & $\begin{array}{l}\frac{1}{6}\left\{3 X_{i j k l m}-\left[\left(X_{i k j l m}+(k l m)\right)+(i j)\right]\right. \\
\left.+\left[X_{k l i j m}+(k l m)\right]\right\}+\frac{1}{6} \delta_{i j}\left[X_{a k a l m}+(k l m)\right] \\
-\frac{1}{10}\left[\left(\delta_{i k} X_{a j a l m}+(k l m)\right)+(i j)\right] \\
-\frac{1}{30}\left[\left(\delta_{i k} X_{a l a m j}+(k l m)\right)+(i j)\right] \\
-\frac{1}{30}\left[\delta_{k l} X_{a m a i j}+(k l m)\right] \\
+\frac{1}{10}\left[\left(\delta_{k l} X_{a i a j m}+(k l m)\right]+(i j)\right] \\
-\frac{1}{15} \delta_{i j} X_{a b a b(k} \delta_{l m)} \\
+\frac{1}{20}\left[X_{a b a b k} \delta_{l(i)} \delta_{j) m}+(k l m)\right]\end{array}$ \\
\hline
\end{tabular}


WEYL MAPS

\begin{tabular}{|l|l|l|l|}
\hline domain of $w$ & image of $w$ & $W=w(T)$ & $T=w^{-1}(W)$ \\
\hline$(4,2) \oplus(2,4)$ & $(4,2) \oplus(2,4)$ & $W_{a b i}=$ & $T_{a b i}=$ \\
$\subset \operatorname{Sym}_{0}^{2} \otimes \mathbb{R}^{4}$ & $\subset \bigwedge^{2} \otimes \mathbb{R}^{4}$ & $-\frac{1}{3}\left(T_{a i b}-(a b)\right)$ & $W_{i a b}+(a b)$ \\
\hline$(5,3) \oplus(3,5)$ & $(5,3) \oplus(3,5)$ & $W_{a b i j}=$ & $T_{a b i j}=$ \\
$\subset \bigwedge^{2}\left(\operatorname{Sym}_{0}^{2}\right)$ & $\subset \operatorname{Sym}_{0}^{2} \otimes \bigwedge^{2}$ & $\frac{1}{4}\left(T_{a i b j}-(i j)\right)$ & {$\left[W_{a i b j}+(a b)\right]+(i j)$} \\
\hline$(5,1) \oplus(1,5)$ & $(5,1) \oplus(1,5)$ & $W_{a b i j}=$ & $T_{a b i j}=$ \\
$\subset \operatorname{Sym}^{2}\left(\operatorname{Sym}_{0}^{2}\right)$ & $\subset \operatorname{Sym}^{2}\left(\bigwedge^{2}\right)$ & $-\frac{1}{3}\left(T_{a i b j}-(a b)\right)$ & $W_{i a b j}+(a b)$ \\
\hline$(6,4) \oplus(4,6)$ & $(6,4) \oplus(4,6)$ & $W_{i j k l m}=$ & $T_{i j k l m}=$ \\
$\subset \operatorname{Sym}_{0}^{2} \otimes \operatorname{Sym}_{0}^{3}$ & $\subset \bigwedge^{2} \otimes \operatorname{Sym}_{0}^{3}$ & $\frac{1}{15}\left[\left(T_{i k j l m}+(k l m)\right)\right.$ & {$\left[\left(W_{i k j l m}+(k l m)\right)\right.$} \\
& & $-(i j)]$ & $+(i j)]$ \\
\hline$(6,2) \oplus(2,6)$ & $(6,2) \oplus(2,6)$ & $W_{i j k l m}=$ & $T_{i j k l m}=$ \\
$\subset \operatorname{Sym}_{0}^{2} \otimes \operatorname{Sym}_{0}^{3}$ & $\subset \operatorname{Sym}^{2}\left(\bigwedge^{2}\right) \otimes \mathbb{R}^{4}$ & $\frac{1}{24}\left[\left(T_{i k j l m}-(i j)\right)\right.$ & {$\left[\left(W_{i k j l m}+(k l m)\right)\right.$} \\
& & $-(k l)]$ & $+(i j)]$ \\
\hline
\end{tabular}

Table 2: For the relations defining the subspaces in columns 1 and 2, consult the text. The components of $T$ and $W$ are ordered alphabetically, and our symmetrization conventions are as in Table 1.

\section{Appendix B. The first eigenvalue of $\Delta_{0}^{A}$}

In this section we establish the lower bound in (8.15). The proof is based on ideas of Taubes [T2].

Theorem B.1. There exist $\lambda_{0}, c_{1}>0$ such that for all $A \in \mathcal{M}_{\lambda_{0}}$ and $v \in$ $\Omega^{0}(\operatorname{Ad} P)$,

$$
\left\|d^{A} v\right\|_{2}^{2} \geq c_{1} \lambda^{2}\|v\|_{2}^{2}
$$

Our argument is based on the principle that if $\left\|d^{A} v\right\|_{2}$ is small compared to $\|v\|_{2}$, then $|v|$ must be nearly constant. Thus the integral of $|v|^{2}$ over any domain $D \subset M$ should be roughly proportional to the volume of $D$. Approximating an instanton $A$ of scale $\lambda$ by the standard instanton (for $\lambda$ small), we show below that $\int_{B_{\lambda}(p)}\left|d^{A} v\right|^{2} \geq c \lambda^{-2} \int_{B_{\lambda}(p)}|v|^{2}$. Since the volume of this ball is essentially $\lambda^{4}$, the principle above then implies that the integral of $\left|d^{A} v\right|^{2}$ over $B_{\lambda}(p)$, and hence over all of $M$, is bounded below by a constant times $\lambda^{2}\|v\|_{2}^{2}$.

We begin with a completely general fact. 
domain $D \subset N$ we have

$$
\int_{D} f^{2} \leq \frac{1}{2} \frac{\operatorname{Vol}(D)}{\operatorname{Vol}(N)}\|f\|_{2}^{2}
$$

then

$$
\|d f\|_{2}^{2} \geq c \operatorname{Vol}(D)^{1-2 / n}\|f\|_{2}^{2} .
$$

(Here and below $\|\cdot\|_{p}$ is the $L^{p}$-norm over all of $N$, and Vol denotes volume of a subset.) The constant $c$ depends only on $\operatorname{Vol}(N)$ and on the first positive eigenvalue of the Laplacian on functions on $N$.

Remark B.1. (1) We emphasize that the domain $D$ above can depend on $f$.

(2) For $n=2$, the lemma is still true if we replace $1 / 2-1 / n$ in (B.2) by an arbitrarily small but positive exponent (with $c$ depending on the exponent); for $n=1$ replace the volume exponent in the corresponding inequality by zero.

Proof. Let us write $V=\operatorname{Vol}(N), V_{D}=\operatorname{Vol}(D)$, and let $f \in L_{1}^{2}(N)$ satisfy $\|f\|_{2}=1$ and inequality (B.1). We will estimate the $L^{2}$ norm of $f$ over $D$ from the identity

$$
V_{D} / V+\int_{D}\left(f^{2}-V^{-1}\right)=\int_{D} f^{2}
$$

By Hölder's inequality we have

$$
\begin{aligned}
\left|\int_{D} f^{2}-V^{-1}\right| & \leq\left\|f-V^{-1 / 2}\right\|_{L^{2 n /(n-2)}(D)}\left\|f+V^{-1 / 2}\right\|_{L^{2}(D)} V_{D}^{1 / n} \\
& \leq\left\|f-V^{-1 / 2}\right\|_{L^{2 n /(n-2)}(N)}\left(\|f\|_{L^{2}(D)}+V^{-1 / 2} V_{D}^{1 / 2}\right) V_{D}^{1 / n}
\end{aligned}
$$

Thus, using (B.1) we have

$$
\left|\int_{D} f^{2}-V^{-1}\right| \leq 2 V^{-1 / 2}\left\|f-V^{-1 / 2}\right\|_{2 n /(n-2)} V_{D}^{1 / 2+1 / n}
$$

Now let $f_{a v}=V^{-1} \int_{N} f$ be the average value of $f$; without loss of generality we may assume $f_{a v} \geq 0$. Since $\int_{N}\left(f-f_{a v}\right)=0$ we have the Poincaré inequality

$$
\left\|f-f_{a v}\right\|_{2}^{2} \leq \mu_{1}^{-1}\|d f\|_{2}^{2}
$$

where $\mu_{1}$ is the first positive eigenvalue of the Laplacian acting on functions on $N$. But $\left\|f-f_{a v}\right\|_{2}^{2}=\|f\|_{2}^{2}-V f_{a v}^{2}=1-V f_{a v}^{2}$, so

$$
\begin{aligned}
0 \leq 1-V^{1 / 2} f_{a v} & \leq\left(1+V^{1 / 2} f_{a v}\right)^{-1} \mu_{1}^{-1}\|d f\|_{2}^{2} \\
& \leq c\|d f\|_{2}^{2}
\end{aligned}
$$

since $f_{a v} \geq 0$. Combining the Sobolev embedding $L_{1}^{2}(N) \hookrightarrow L^{2 n /(n-2)}(N)$ with (B.5) we have

$$
\left\|f-f_{a v}\right\|_{2 n /(n-2)} \leq c\|d f\|_{2}
$$


and thus

$$
\left\|f-V^{-1 / 2}\right\|_{2 n /(n-2)} \leq c\left(\|d f\|_{2}+\|d f\|_{2}^{2}\right)
$$

by the triangle inequality and the bounds (B.6- B.7). Returning to (B.4) we then find

$$
\left|\int_{D} f^{2}-V^{-1}\right| \leq c V_{D}^{1 / 2+1 / n}\left(\|d f\|_{2}+\|d f\|_{2}^{2}\right) .
$$

Inserting this into (B.3) then gives us

$$
V_{D} / V-c V_{D}^{1 / 2+1 / n}\left(\|d f\|_{2}+\|d f\|_{2}^{2}\right) \leq \int_{D} f^{2} \leq \frac{1}{2} V_{D} / V .
$$

Writing $\alpha=1 / 2-1 / n$, the above inequality implies

$$
c V_{D}^{\alpha} \leq\|d f\|_{2}+\|d f\|_{2}^{2} \leq \frac{1}{2} c V_{D}^{\alpha}+\left(\frac{1}{2}\left(c V_{D}\right)^{-\alpha}+1\right)\|d f\|_{2}^{2}
$$

(where in this line $c$ has the same value in all three appearances). Rearranging this inequality and noting that $V_{D}$ is bounded from above by $V$, (B.2) follows.

Applying this to geodesic balls of radius $\lambda$ in the four-dimensional manifold $M$ we immediately have the following.

Corollary B.3. There exist positive constants $c_{2}, c_{3}$ such that if $p \in M, \lambda \in$ $R, f \in L_{1}^{2}(M)$, and

$$
\int_{B_{\lambda}(p)} f^{2} \leq c_{2} \lambda^{4}\|f\|_{2}^{2}
$$

then

$$
\|d f\|_{2}^{2} \geq c_{3} \lambda^{2}\|f\|_{2}^{2}
$$

This quantifies the principle mentioned earlier. The second ingredient is the next lemma.

Lemma B.4. There exist $\lambda_{0}, c_{4}>0$ such that if $A \in \mathcal{M}_{\lambda_{0}}$ has scale $\lambda$ and center $p$, and if $v \in \Omega^{0}(\operatorname{Ad} P)$, then

$$
\int_{B_{\lambda}(p)}\left|d^{A} v\right|^{2} \geq c_{4} \lambda^{-2} \int_{B_{\lambda}(p)}|v|^{2} .
$$

Proof. Let $g_{\lambda},\left\{y^{i}\right\}$ be as in $\S 3$ and let $B=B_{\lambda}(p)$. We then have

$$
\int_{B}\left|d^{A} v\right|_{g}^{2} \Omega(g)=\lambda^{-2} \int_{B}\left|d^{A} v\right|_{g_{\lambda}}^{2} \Omega\left(g_{\lambda}\right) .
$$

Using the coordinates $\left\{y^{i}\right\}$ to pull the problem back to $\mathbb{R}^{4}$, the integral on the right is taken simply over the unit ball $B_{1} \subset \mathbb{R}^{4}$, and $g_{\lambda}$ and its volume form can be made arbitrarily close to the flat metric and its volume form by taking $\lambda$ sufficiently small. If $A_{1}$ is the standard instanton on $\mathbb{R}^{4}$, then given $\epsilon$ there exists $\lambda_{0}$ such that $A \in \mathcal{M}_{\lambda_{0}}$ implies $\left\|A-A_{1}\right\|_{C^{\circ}\left(B_{1}\right)} \leq \epsilon([\mathrm{D} 1$, Theorem 
16.(ii)]); here the norm is taken with respect to the flat metric. For such $A$ we therefore have

$$
\int_{B}\left|d^{A} v\right|_{g_{\lambda}}^{2} \Omega\left(g_{\lambda}\right) \geq \frac{1}{2} \int_{B_{1}}\left|d^{A_{1}} v\right|^{2} d^{4} y-\epsilon^{2} \int_{B}|v|^{2} d^{4} y
$$

where $\left|d^{A_{1}} v\right|$ is taken in the flat metric.

Now suppose that

$$
\inf _{L_{2}^{1}\left(B_{1}\right)} \frac{\int_{B_{1}}\left|d^{A_{1}} v\right|^{2} d^{4} y}{\int_{B_{1}}|v|^{2} d^{4} y}=0 .
$$

Taking a minimizing sequence and using the Rellich theorem we find a smooth section $v_{0} \neq 0$ over $B_{1}$ such that $d^{A} v_{0} \equiv 0$, implying $\left[F^{A_{1}}, v_{0}\right]=d^{A_{1}} d^{A_{1}} v_{0} \equiv 0$ as well. But the standard instanton $A_{1}$ has the property that at each $y \in B_{1}$ and $0 \neq w \in \operatorname{Ad} P_{y},\left[F^{A_{1}}(y), w\right] \neq 0$ (see, for example, the formula for $F^{A_{1}}$ in [FU, eq. (6.6)]). This implies the contradiction $v_{0} \equiv 0$, so the infimum in (B.11) must be positive. Thus, taking $\epsilon$ small enough in (B.10) and using (B.9), the bound (B.8) follows.

Proof of Theorem B.1. Let $\lambda_{0}, c_{2}, c_{3}, c_{4}$ be as in Corollary B.3 and Lemma B.4. Suppose $A \in \mathcal{M}_{\lambda_{0}}, v \in \Omega^{0}(\operatorname{Ad} P)$, and

$$
\left\|d^{A} v\right\|_{2}^{2}<c_{3} \lambda^{2}\|v\|_{2}^{2} \text {. }
$$

Since $|d| v|| \leq\left|d^{A} v\right|$, Corollary B.3 applied to $f=|v|$ implies

$$
\int_{B_{\lambda}(p)}|v|^{2}>c_{2} \lambda^{4}\|v\|_{2}^{2}
$$

But then

$$
\left\|d^{A} v\right\|_{2}^{2} \geq \int_{B_{\lambda}(p)}\left|d^{A} v\right|^{2} \geq c_{4} \lambda^{-2} \int_{B_{\lambda}(p)}|v|^{2} \geq c_{2} c_{4} \lambda^{2}\|v\|_{2}^{2} .
$$

Thus for all $v$ we have $\left\|d^{A} v\right\|_{2}^{2} \geq \min \left(c_{3}, c_{2} c_{4}\right) \lambda^{2}\|v\|_{2}^{2}$.

\section{REFERENCES}

[DMM] Doi, H., Matsumoto, Y. and Matumoto, T., An explicit formula of the metric on the moduli space of BPST-instantons over $\mathfrak{S}^{4}$ in A Fête of Topology, Academic Press 1987.

[D1] Donaldson, S. K., An application of gauge theory to four-dimensional topology, J. Diff. Geom. 18 (1983), 279-315.

[D2] Compactification and completion of Yang-Mills moduli spaces In Lecture Notes in Mathematics 1410 (Differential Geometry: Proceedings of the 3rd International Symposium, held at Peniscola, Spain, June 5-12, 1988), ed. Carreras et. al., Springer-Verlag, Berlin, Heidelberg, New York 1989.

[DK] Donalson, S. K. and Kronheimer, P., The Geometry of Four-Manifolds, Oxford University Press, New York 1990. 
[FU] Freed, D. S. and Uhlenbeck, K. K., Instantons and Four-Manifolds, SpringerVerlag, Berlin, Heidelberg, New York, Tokyo 1984.

[G1] Groisser, D., The geometry of the moduli space of $\mathfrak{C P}^{2}$ instantons, Inventiones Mathematicae, 99 (1990), 393-409.

[G2] - Curvature of Yang-Mills moduli spaces near the boundary, II, In preparation.

[GP1] Groisser, D. and Parker, T. H., The Riemannian geometry of the Yang-Mills moduli space, Commun. Math. Phys. 112 (1987), 663-689.

[GP2] - The geometry of the Yang-Mills moduli space for definite manifolds, J. Differential Geom. 29 (1989).

[GP3] , Semiclassical Yang-Mills theory I: Instantons, Commun. Math. Phys. 135 (1990), 101-140.

[GT] Gilbarg, D. and Trudinger, N. S. Elliptic Partial Differential Equations of Second Order, second edition. Springer-Verlag, Berlin, New York 1983.

[H] Habermann, L., On the geometry of the space of Sp(1)-instantons with Pontrjagin index 1 on the 4-sphere, Ann. Global Anal. Geom., 6 (1988), 3-29.

[Ka] Kato, T., Perturbation Theory for Linear Operators second edition, SpringerVerlag, Berlin, Heidelberg, New York 1976.

[Ko] Kobayashi, K., Three Riemannian metrics on the moduli space of 1-instantons over $\mathbb{C P}^{2}$, Hiroshima Math. J. 19 (1989), 243-249.

[L] Lawson, H.B., The theory of gauge fields in four dimensions, Amer. Math. Soc., Providence, RI 1986.

[T1] Taubes, C.H., A Framework for Morse Theory for the Yang-Mills Functional, Invent. Math.94 (1988), 327-402.

[T2] , private communication, ca. 1986.

University of Florida, U. S. A.

Received September 10, 1992. Revised January 20, 1993. 
